SUPPORTING INFORMATION

\title{
Does Cation Size Affect Occupancy and Electrostatic Screening of the Nucleic Acid Ion Atmosphere?
}

\author{
Magdalena Gebala $^{1}$, Steve Bonilla ${ }^{2}$, Namita Bisaria ${ }^{1, \dagger}$, Daniel Herschlag ${ }^{1,3,4^{*}}$
}

1) Department of Biochemistry, Stanford University, Stanford, California, 94305 USA

2) Department of Chemical Engineering, Stanford University, Stanford, California, 94305 USA

3) Department of Chemistry, Stanford University, Stanford, California, 94305 USA

4) ChEM-H Institute, Stanford University, Stanford, California, 94305 USA

${ }^{\dagger}$ Current address: Whitehead Institute for Biomedical Research, Cambridge, MA 02142

*Corresponding author: herschla@stanford.edu

Table of Contents

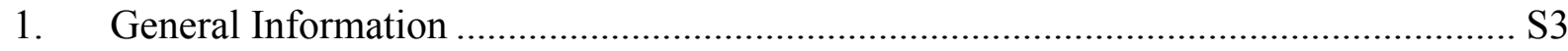

2. Conversion of molar concentration units to molal concentration units and calculation

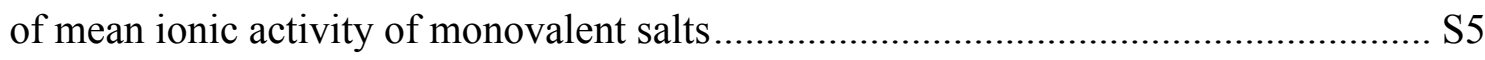

3. Figure S1. smFRET results represented as a function of the acquisition rate .............. S5

4. Figures S2-S15. smFRET data assessment of P4-P6 RNA folding as follows: determination of $k_{\text {fold }}$ and $k_{\text {unfold }}$ rate constants; scatter plots of ${ }_{k f o l d}$ versus kunfold rate constants; kfold and kunfold rate constants as a function of signal-to-noise ratio in the donor and acceptor channel; histograms of trace lengths; histograms of $\Delta \mathrm{G}$ values; the bulk equilibrium; distribution of the number of transitions per trace.

5. Figure S16. Competitive association of $\mathrm{Na}^{+}$against $16 \mathrm{mM}$ and $50 \mathrm{mM} \mathrm{Li}^{+}$around 24-bp DNA

6. Figure S17. Comparison between the experimentally determined $\alpha$ values and 3D-RISM computational estimated of the $\alpha$ values for ions around 24-bp DNA and 24-bp RNA 
7. Figure S18. Cation competition between $\mathrm{Na}^{+}$and $\mathrm{Cs}^{+}$in the presence of $\mathrm{Cl}^{-}$and comparison to cation competition between $\mathrm{Na}^{+}$and $\mathrm{Cs}^{+}$in the presence of $\mathrm{Br}^{-}$........ S50

8. Figure S19. Comparison of prior experimental results (ref 1) of $\mathrm{TMA}^{+}$and $\mathrm{Na}^{+}$

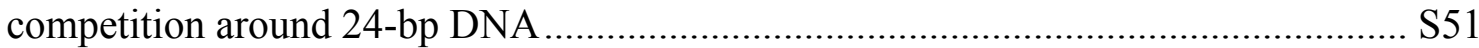

9. Figure S20. Competitive $\mathrm{Cs}^{+}$association for the 24-bp DNA as a function of $\mathrm{Na}^{+}$

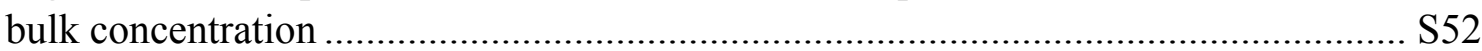

10. Figure S21. Competitive $\mathrm{Cs}^{+}$association for the 24-bp DNA as a function of solution mean activity S53

11. Figure S22. Competitive association of $\mathrm{Li}^{+}$and $\mathrm{Cs}^{+}$for the 24-bp DNA against $\mathrm{Na}^{+}$as a function of an anion

12. Figure S23. Prior data for salt-mediated relaxation of a DNA helix-junction-helix as a function of salt activity coefficients

13. Table S1. Experimentally determined preferential interaction coefficients and $\alpha$ value for $\mathrm{LiBr}$ around 24-bp DNA in the presence of $40 \mathrm{mM} \mathrm{NaBr}$

14. Table S2. Experimentally determined preferential interaction coefficients and $\alpha$ value for $\mathrm{KBr}$ around 24-bp DNA in the presence of $50 \mathrm{mM} \mathrm{NaBr}$

15. Table S3: Experimentally determined preferential interaction coefficients and $\alpha$ value for $\mathrm{RbBr}$ around 24-bp DNA in the presence of $50 \mathrm{mM} \mathrm{NaBr}$

16. Table S4: Experimentally determined preferential interaction coefficients and $\alpha$ value for $\mathrm{CsBr}$ around 24-bp DNA in the presence of $50 \mathrm{mM} \mathrm{NaBr}$

17. Table S5: Experimentally determined preferential interaction coefficients and $\alpha$ value for TMABr around 24-bp DNA in the presence of $50 \mathrm{mM} \mathrm{NaBr}$

18. Table S6: Experimentally determined preferential interaction coefficients and $\alpha$ value for TBABr around 24-bp DNA in the presence of $40 \mathrm{mM} \mathrm{NaBr}$

19. Table S7: Summary of experimentally determined cation competition constant, $\alpha$ value, against $\mathrm{NaBr}$ around 24-bp DNA (Table S1-S6)

20. Table S8: Experimentally determined preferential interaction coefficients for $\mathrm{LiBr}$ around 24-bp DNA at difference salt concentrations

21. Table S9: Experimentally determined preferential interaction coefficients and $\alpha$ value $\mathrm{NaBr}$ around 24-bp DNA in the presence of $16 \mathrm{mM} \mathrm{LiBr}$.....

22. Table S10: Experimentally determined preferential interaction coefficients and $\alpha$ value for $\mathrm{NaBr}$ around 24-bp DNA in the presence of $50 \mathrm{mM} \mathrm{LiBr}$

23. Table S11: Experimentally determined preferential interaction coefficients and $\alpha$ value for $\mathrm{CsBr}$ around 24-bp DNA in the presence of $20 \mathrm{mM} \mathrm{NaBr}$

24. Table S12: Experimentally determined preferential interaction coefficients and $\alpha$ value for $\mathrm{CsBr}$ around 24-bp DNA in the presence of $150 \mathrm{mM} \mathrm{NaBr}$.

25. Table S13: Experimentally determined preferential interaction coefficients for $\mathrm{CsBr}$ around 24-bp DNA in the presence of $300 \mathrm{mM} \mathrm{NaBr}$

26. Table S14: Experimentally determined preferential interaction coefficients for CsF around 24-bp DNA in the presence of $300 \mathrm{mM} \mathrm{NaF}$

27. Table S15: Experimentally determined preferential interaction coefficients and $\alpha$ value for $\mathrm{LiBr}$ around 24-bp DNA in the presence of $5 \mathrm{mM} \mathrm{MgBr} 2$ 
28. Table S16: Experimentally determined preferential interaction coefficients and $\alpha$ value for $\mathrm{NaBr}$ around 24-bp DNA in the presence of $6 \mathrm{mM} \mathrm{MgBr}_{2}$

29. Table S17: Experimentally determined preferential interaction coefficients and $\alpha$ value for $\mathrm{CsBr}$ around 24-bp DNA in the presence of $6 \mathrm{mM} \mathrm{MgBr}_{2}$

30. Table S18: Experimentally determined preferential interaction coefficients and $\alpha$ value for $\mathrm{CsBr}$ around 24-bp RNA in the presence of $50 \mathrm{mM} \mathrm{NaBr}$

31. Table S19: Experimentally determined preferential interaction coefficients and $\alpha$ value for $\mathrm{LiCl}$ around 24-bp DNA in the presence of $60 \mathrm{mM} \mathrm{NaO} \mathrm{As}_{2}\left(\mathrm{CH}_{3}\right)_{2}$

32. Table S20. Experimentally determined preferential interaction coefficients and $\alpha$ value for $\mathrm{CsBr}$ around 24-bp DNA in the presence of $40 \mathrm{mM} \mathrm{NaO}_{2} \mathrm{As}\left(\mathrm{CH}_{3}\right)_{2}$

33. Table S21. Kinetic and thermodynamic parameter for P4-P6 RNA folding in the presence of $\mathrm{NaCl}$

34. Table S22. Kinetic and thermodynamic parameter for P4-P6 RNA folding in the presence of $\mathrm{KCl}$

35. Table S23. Kinetic and thermodynamic parameter for P4-P6 RNA folding in the presence of $\mathrm{RbCl}$

36. Table S24. Kinetic and thermodynamic parameter for P4-P6 RNA folding in the presence of $\mathrm{RbF}$

37. Table S25. Mean activity coefficients $\left(\gamma_{ \pm}\right)$of $\mathrm{NaX}, \mathrm{KCl}, \mathrm{RbX}$ and $\mathrm{CsX}$ salts at $2.0 \mathrm{M}$ and $25^{\circ} \mathrm{C}$ S64

38. References S64 


\section{General Information}

Control experiments including the following were published in ref 2 (Supporting Information): i) the dependence and accuracy of the ion counting method on DNA concentration; ii) the equilibration between DNA-containing samples and the buffer; iii) the linear range and precision of the ICP MS spectrometer; and iv) the effects of sample dilution on measurements precision.

In cases where a cation or an anion could not be assayed by ICP MS (see Experimental Methods in main text), the number of accumulated cations and excluded anions was calculated based on the charge neutrality principle from the measured number of depleted anions and the total charge of 24-bp DNA (eq 1, main text), as established by prior results. ${ }^{2}$

Previous studies on the relaxation of short DNA helices attached by a short, flexible linker showed a correlation between electrostatic screening of the nucleic acid potential and ion size. ${ }^{3}$ However, these studies required very high salt concentrations and recent ion counting results ${ }^{2}$ revealed non-ideality effects between cations and anions under high-salt conditions. Specifically, high monovalent salt concentrations lead to ion complex formation that depends on the identity of the anion present, and this property affects the ability and tendency of cations to occupy the ion atmosphere. We tested this activity model initially by cation competition experiments. We observed that at high salt concentrations the monovalent cation occupancy within the ion atmosphere and hence its ability to compete another monovalent cation depends on the nature of the accompanying anion; a discussion of these results is presented in the main text ("Evidence for an alternative model for cation-size effects at high salt concentrations"). In contrast, we observed that the cation occupancy in the ion atmosphere is independent of the accompanying anion at low and moderate (i.e., $<100 \mathrm{mM}$ ) bulk concentrations, in accord with the activity model (Figures S22) and prior results. ${ }^{2}$ Specifically, no variation in the cation occupancy was observed in the presence of $\mathrm{Cl}^{-}, \mathrm{Br}^{-}$and $\left(\mathrm{CH}_{3}\right)_{2} \mathrm{AsO}_{2}{ }^{-}$(Figure 22C). Subsequently, we reevaluated the prior relaxation results as a function of salt activity, which reflects the nonideality effects of simple electrolyte solutions, instead of salt concentration. Results from this analysis are shown in Figure S23. 
Conversion of molar concentration units to molal concentration units and calculation of mean ionic activity of monovalent salts: Molar concentrations were converted to molal concentrations according to: ref 4

$$
m=C /\left(d_{s}-0.001 \cdot M_{W}\right)
$$

where $m$ is molal concentration, $C$ is molar concentration, $d_{s}$ is density of sample and $M_{W}$ is molecular weight of water $(18.01 \mathrm{~g} / \mathrm{mol})$. Densities $\left(\rho_{\mathrm{s}}\right)$ of salt solutions were obtained based on gravimetric measurements according to:

$$
\rho_{s}=\frac{m_{s}}{V_{s}}
$$

where $m_{s}$ is measured solution mass, $V_{s}$ is sample volume $(20 \mu \mathrm{L})$.

Mean ionic activity $\left(\mathrm{a}_{ \pm}\right)$of monovalent salts were estimated based on:

$$
a_{ \pm}=m \cdot \gamma_{ \pm}
$$

where $m$ is molal concentration and $\gamma_{ \pm}$is mean activity coefficient and data were from ref 5 .

\section{Supporting Figures:}
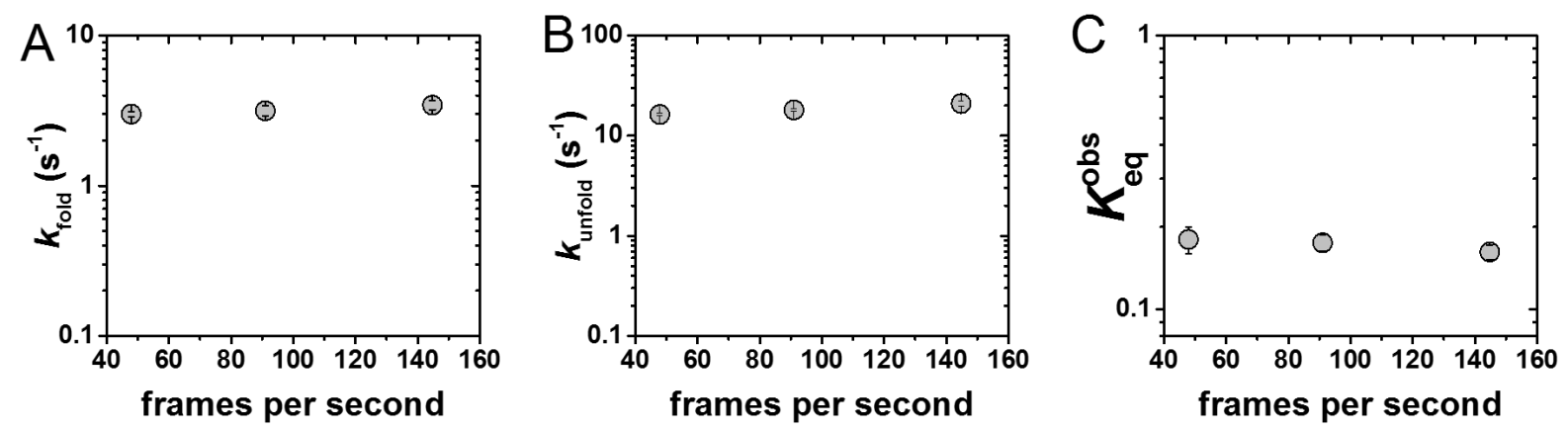

Figure S1. smFRET results represented as a function of the acquisition rate. A) Folding rates, B) unfolding rates, and $C$ ) equilibrium constants acquired at various frame frequencies: 48, 92 and 145 frame per second. Experimental conditions; $1.8 \mathrm{M} \mathrm{NaCl}$ in $40 \mathrm{mM}$ Na-MOPS, pH 7.0, and 0.1 mM Na-EDTA. 
A

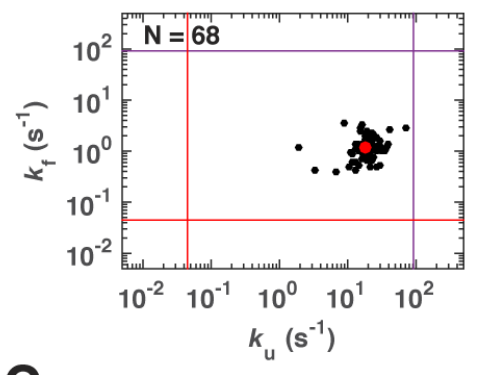

C

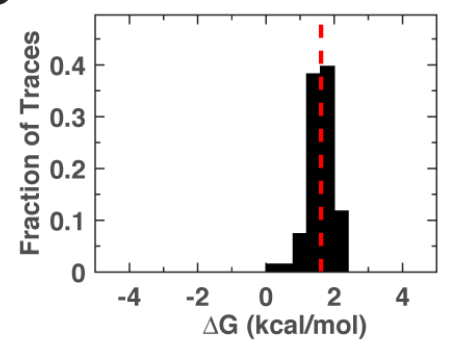

$\mathbf{E}$

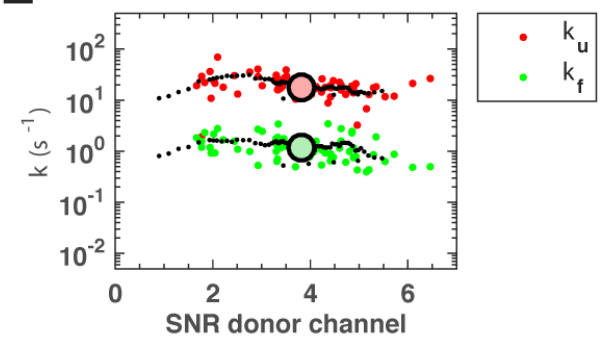

B

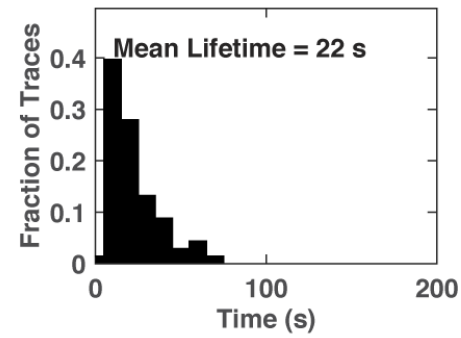

D

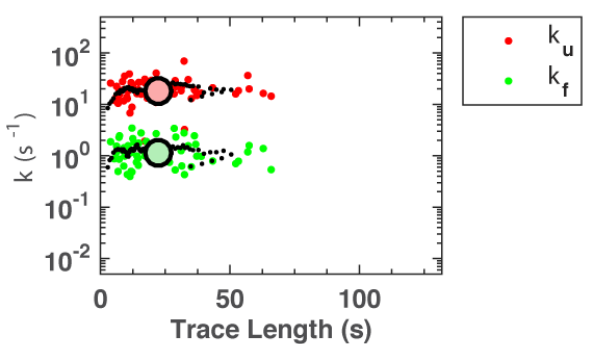

$\mathbf{F}$

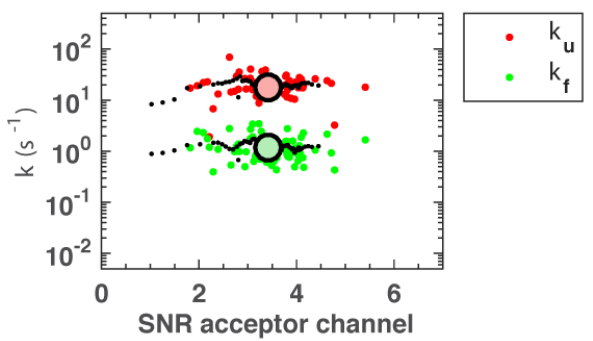

Figure S2-1. smFRET data for P4-P6 A225U in 1.5 M NaCl. A) Scatter plot of fitted values of $k_{\mathrm{f}}$ versus $k_{\mathrm{u}}$ for each molecule. ${ }^{6}$ Purple lines indicate the camera frame rate and red lines indicate the average lifetime of the molecules. The red dot indicates mean folding and unfolding rate constants. TIRF measurements were taken at 91 frames per second (see Experimental Methods in main text). $\mathrm{N}=$ number of molecules. B) Histogram of trace lengths for individual molecules. C) Histogram of $\Delta \mathrm{G}$ values for individual molecules (determined from equilibrium $K_{e q}=k_{f} / k_{u}$ ). The mean value is denoted by the dashed red line. D) Scatter plot of rate constants versus trace length; $k_{\mathrm{f}}$ (green) and $k_{\mathrm{u}}$ (red). The mean value for each rate constant is presented by larger light green $\left(k_{\mathrm{f}}\right)$ and red $\left(k_{\mathrm{u}}\right)$ circles. The black dots are the average of ten values and are shown to provide an empirical guide. E) and F) Rate constants as a function of signal-to-noise ratio (SNR) in the donor channel and acceptor channels respectively. Colors as in (D). 
A
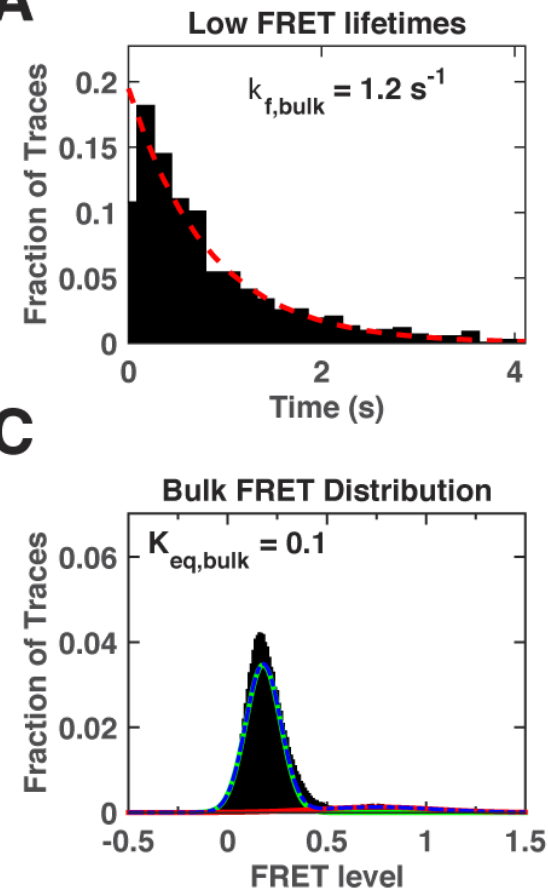
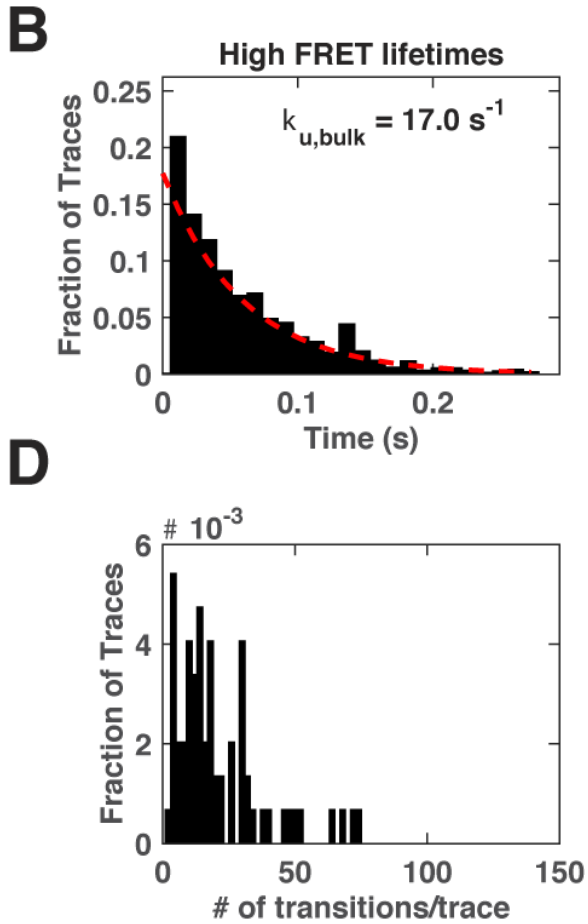

Figure S2-2. smFRET data of P4-P6 variant A225U in 1.5 M NaCl. A) and B) Distribution of the $k_{\mathrm{f}}$ (folding) and $k_{\mathrm{u}}$ (unfolding) rate constants as a function of time. An exponential fit (dashed red lines) to the distribution of lifetimes in the unfolded (A) or folded (B) states of all molecules was used to determine $k_{\mathrm{f}}(\mathrm{A})$ and $k_{\mathrm{u}}(\mathrm{B})$ rate constants (see Experimental Methods in main text). C) Histogram of FRET distribution for all molecules. Distribution is fit to a two-Gaussian model (blue). The equilibrium constant $\left(K_{\text {eq,bulk }}\right)$ was determined from the ratio of the fraction of molecules that are in the high FRET peak (red) versus low FRET peak (green). D) Distribution of the number of transitions per trace. 

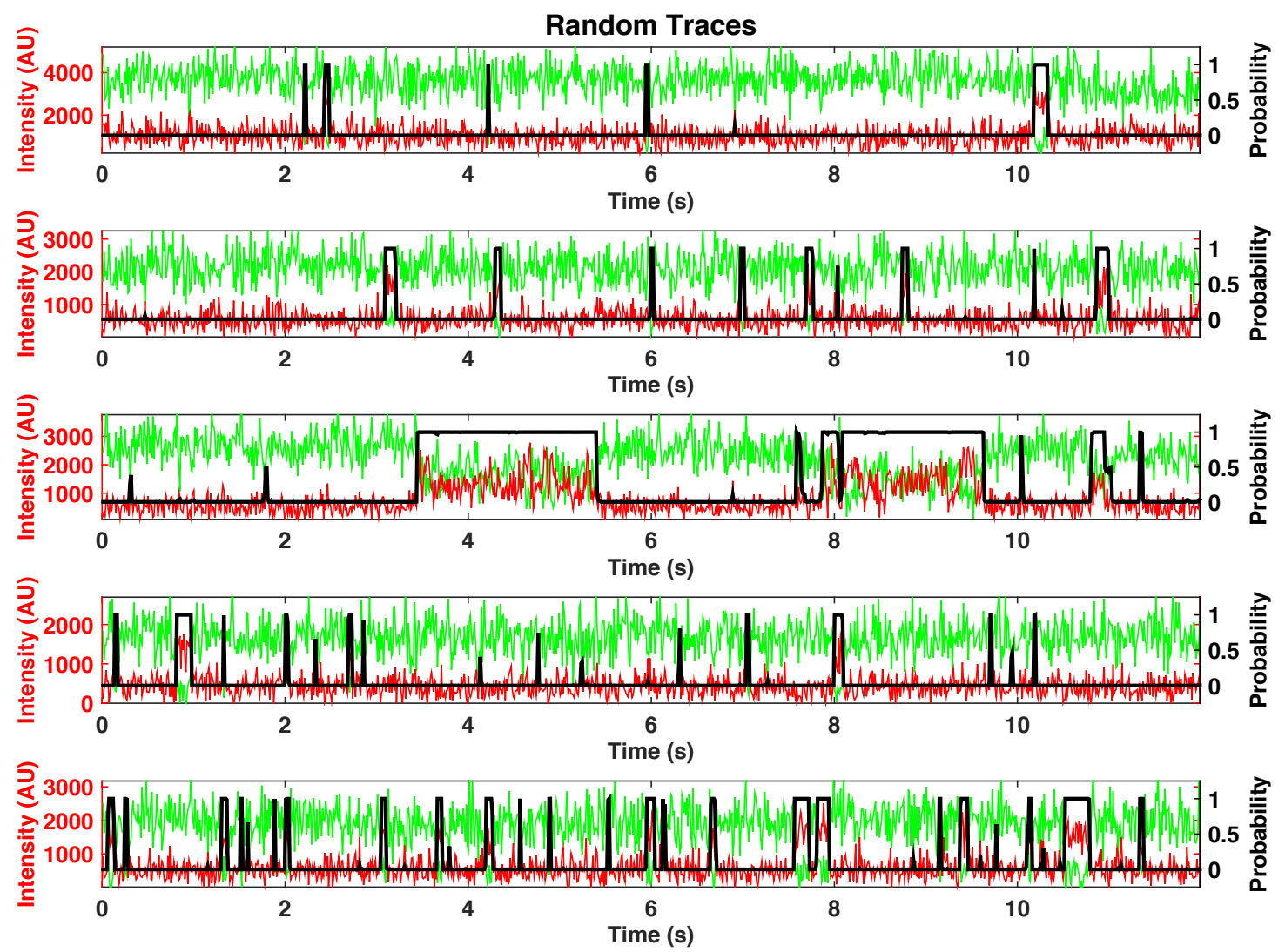

Figure S2-3. Randomly selected FRET traces of P4-P6 variant A225U in $1.5 \mathbf{~ M ~ N a C l}$. The intensities of the donor dye (green) and the acceptor dye (red) are shown. The black line denotes the probability of the high FRET state determined by a hidden Markov model fit to the data. ${ }^{6}$ To scale the length of the randomly selected FRET traces and ease their comparison, traces were truncated at the length of the shortest selected trace. 
A

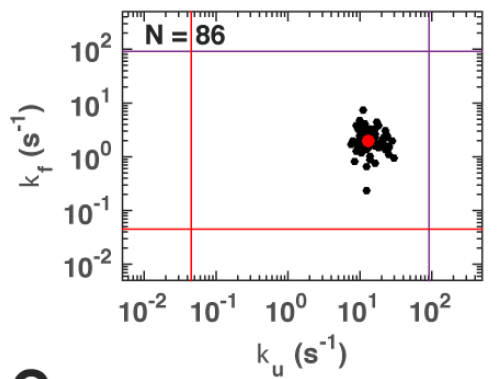

C

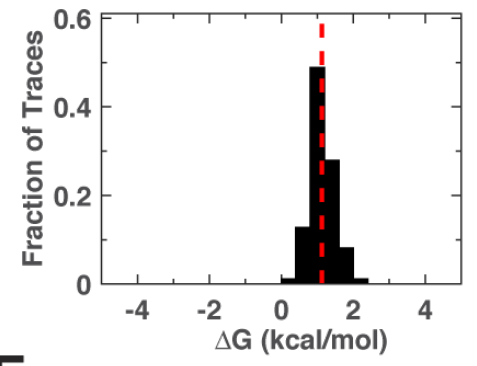

E

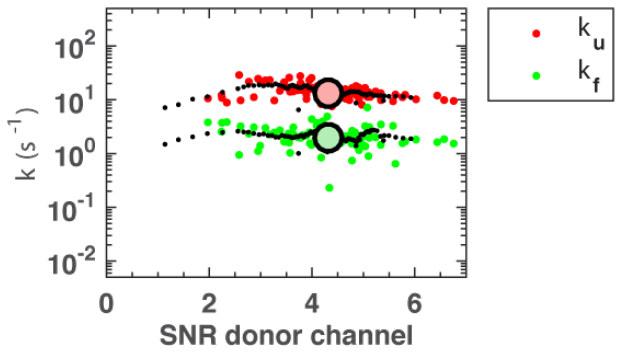

B

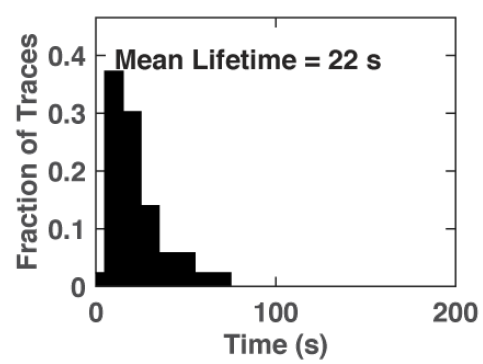

D

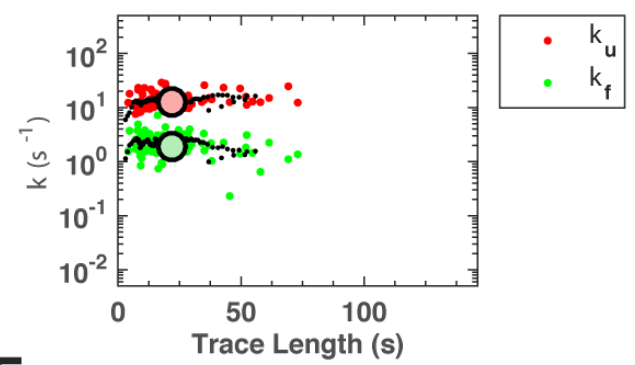

$\mathbf{F}$

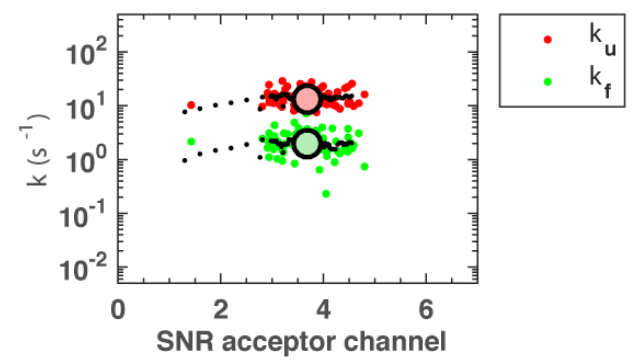

Figure S3-1. smFRET data for P4-P6 A225U in 1.9 M NaCl. A) Scatter plot of fitted values of $k_{\mathrm{f}}$ versus $k_{\mathrm{u}}$ for each molecule. ${ }^{6}$ Purple lines indicate the camera frame rate and red lines indicate the average lifetime of the molecules. The red dot indicates mean folding and unfolding rate constants. TIRF measurements were taken at 91 frames per second (see Experimental Methods in main text). $\mathrm{N}=$ number of molecules. B) Histogram of trace lengths for individual molecules. C) Histogram of $\Delta \mathrm{G}$ values for individual molecules (determined from equilibrium $K_{e q}=k_{f} / k_{u}$ ). The mean value is denoted by the dashed red line. D) Scatter plot of rate constants versus trace length; $k_{\mathrm{f}}$ (green) and $k_{\mathrm{u}}$ (red). The mean value for each rate constant is presented by larger light green $\left(k_{\mathrm{f}}\right)$ and red $\left(k_{\mathrm{u}}\right)$ circles. The black dots are the average of ten values and are shown to provide an empirical guide. E) and F) Rate constants as a function of signal-to-noise ratio (SNR) in the donor channel and acceptor channels respectively. Colors as in (D). 
A
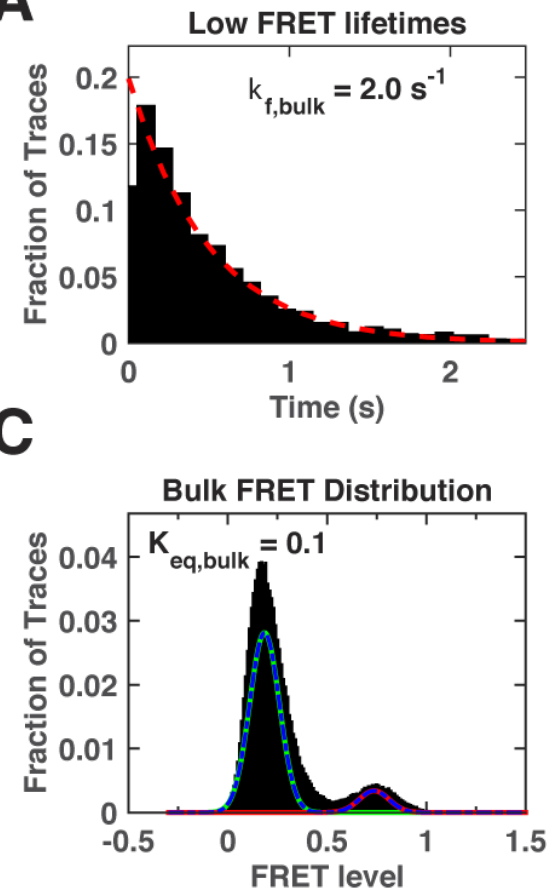

B

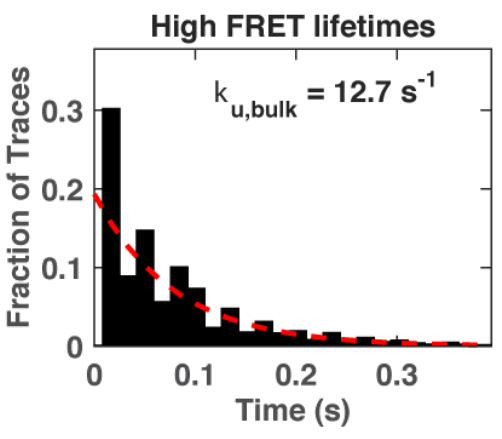

D

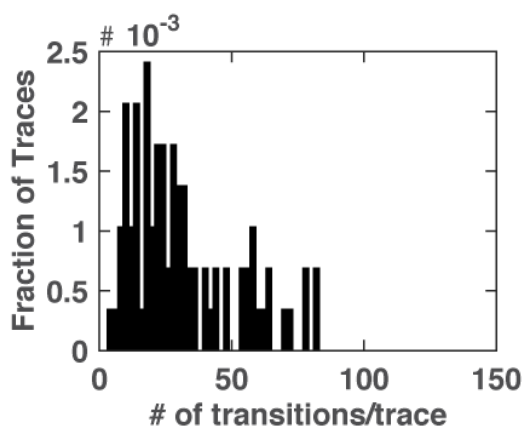

Figure S3-2. smFRET data of P4-P6 variant A225U in 1.9 M NaCl. A) and B) Distribution of the $k_{\mathrm{f}}$ (folding) and $k_{\mathrm{u}}$ (unfolding) rate constants as a function of time. An exponential fit (dashed red lines) to the distribution of lifetimes in the unfolded (A) or folded (B) states of all molecules was used to determine $k_{\mathrm{f}}(\mathrm{A})$ and $k_{\mathrm{u}}(\mathrm{B})$ rate constants (see Experimental Methods in main text). C) Histogram of FRET distribution for all molecules. Distribution is fit to a two-Gaussian model (blue). The equilibrium constant $\left(K_{\text {eq,bulk }}\right)$ was determined from the ratio of the fraction of molecules that are in the high FRET peak (red) versus low FRET peak (green). D) Distribution of the number of transitions per trace. 

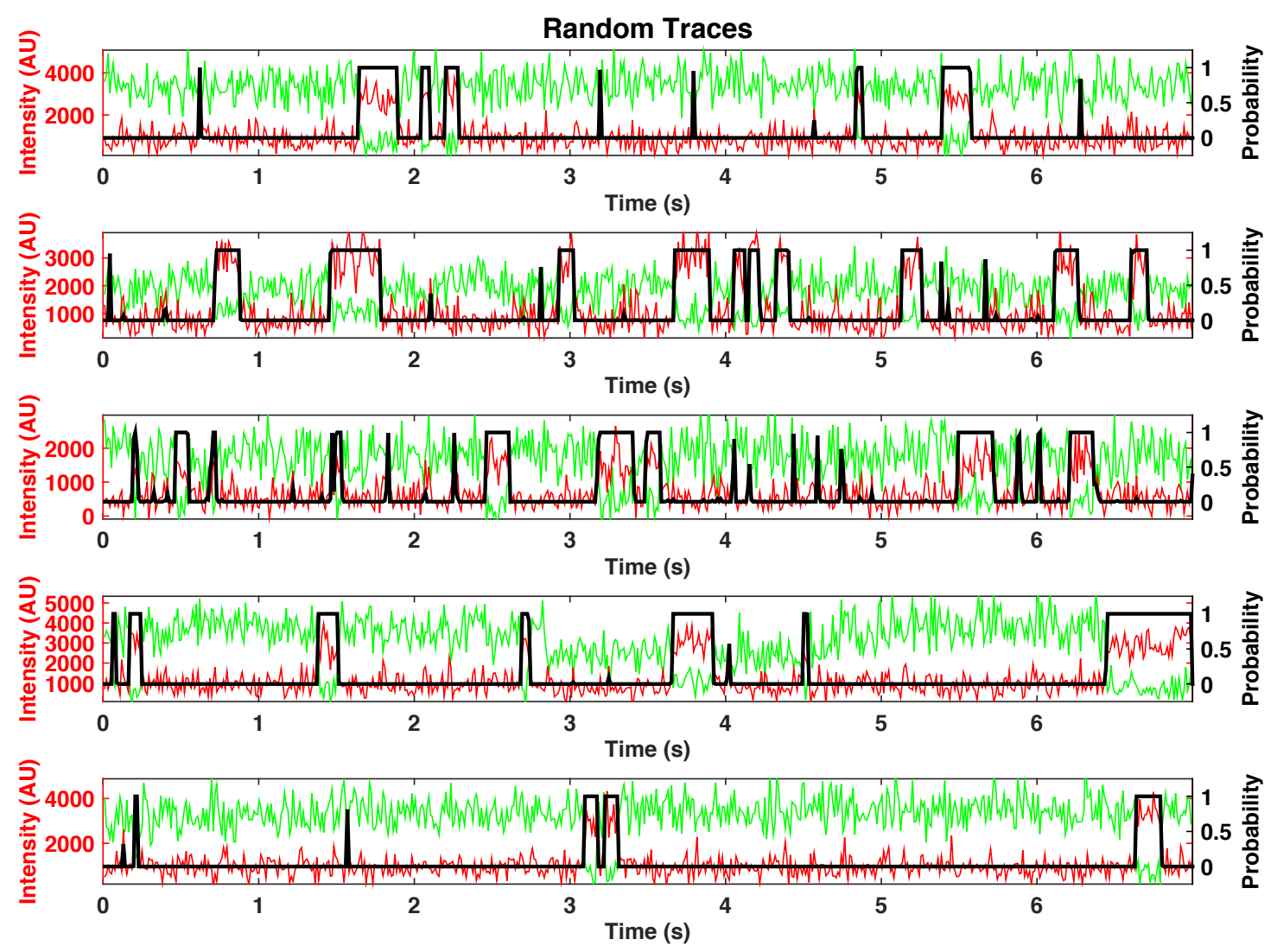

Figure S3-3. Randomly selected FRET traces of P4-P6 variant A225U in 1.9 M NaCl. The intensities of the donor dye (green) and the acceptor dye (red) are shown. The black line denotes the probability of the high FRET state determined by a hidden Markov model fit to the data. ${ }^{6}$ To scale the length of the randomly selected FRET traces and ease their comparison, traces were truncated at the length of the shortest selected trace. 

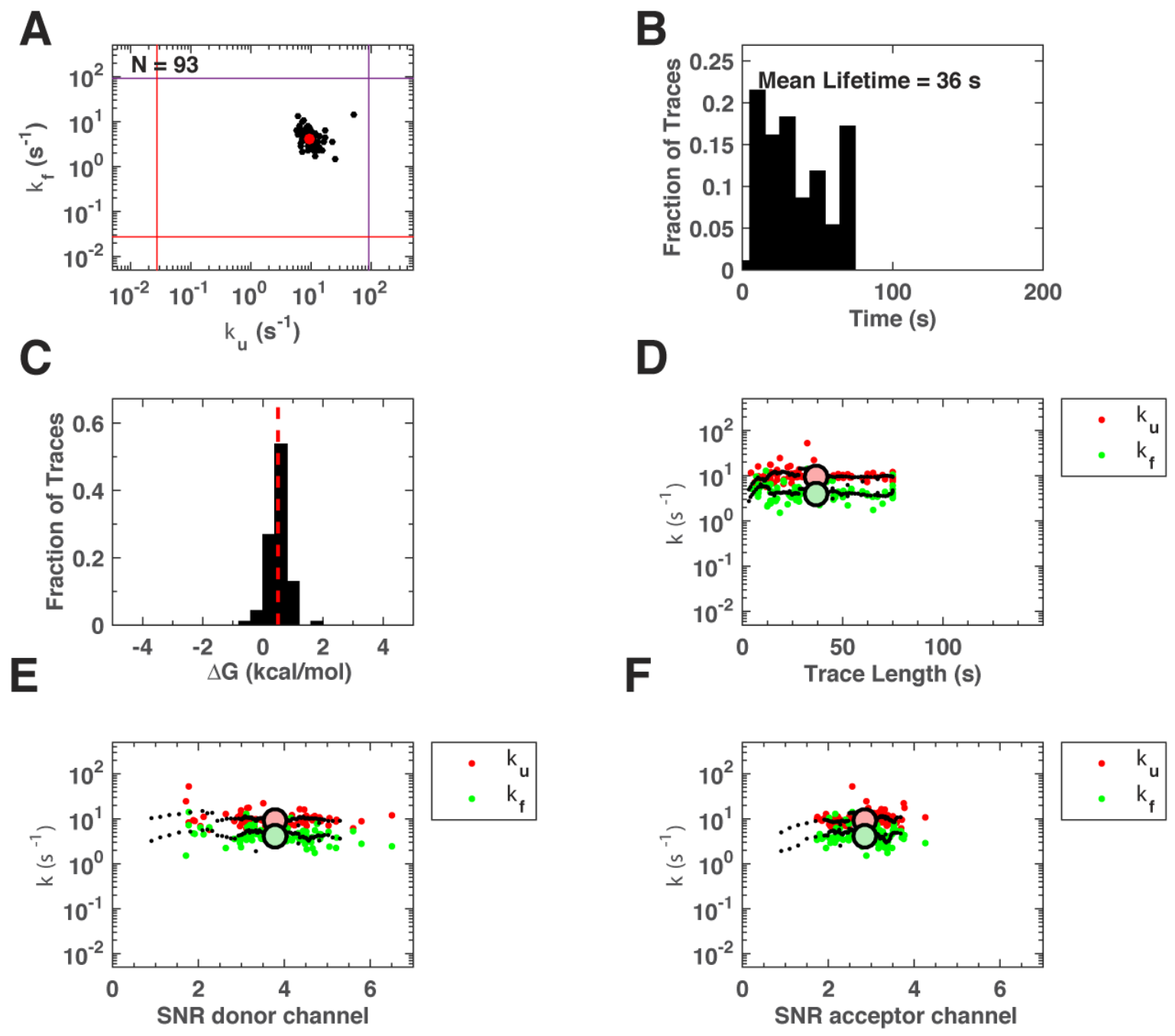

Figure S4-1. smFRET data for P4-P6 A225U in 2.4 M NaCl. A) Scatter plot of fitted values of $k_{\mathrm{f}}$ versus $k_{\mathrm{u}}$ for each molecule. ${ }^{6}$ Purple lines indicate the camera frame rate and red lines indicate the average lifetime of the molecules. The red dot indicates mean folding and unfolding rate constants. TIRF measurements were taken at 91 frames per second (see Experimental Methods in main text). $\mathrm{N}=$ number of molecules. B) Histogram of trace lengths for individual molecules. C) Histogram of $\Delta \mathrm{G}$ values for individual molecules (determined from equilibrium $K_{e q}=k_{f} / k_{u}$ ). The mean value is denoted by the dashed red line. D) Scatter plot of rate constants versus trace length; $k_{\mathrm{f}}$ (green) and $k_{\mathrm{u}}$ (red). The mean value for each rate constant is presented by larger light green $\left(k_{\mathrm{f}}\right)$ and red $\left(k_{\mathrm{u}}\right)$ circles. The black dots are the average of ten values and are shown to provide an empirical guide. E) and F) Rate constants as a function of signal-to-noise ratio (SNR) in the donor channel and acceptor channels respectively. Colors as in (D). 
A
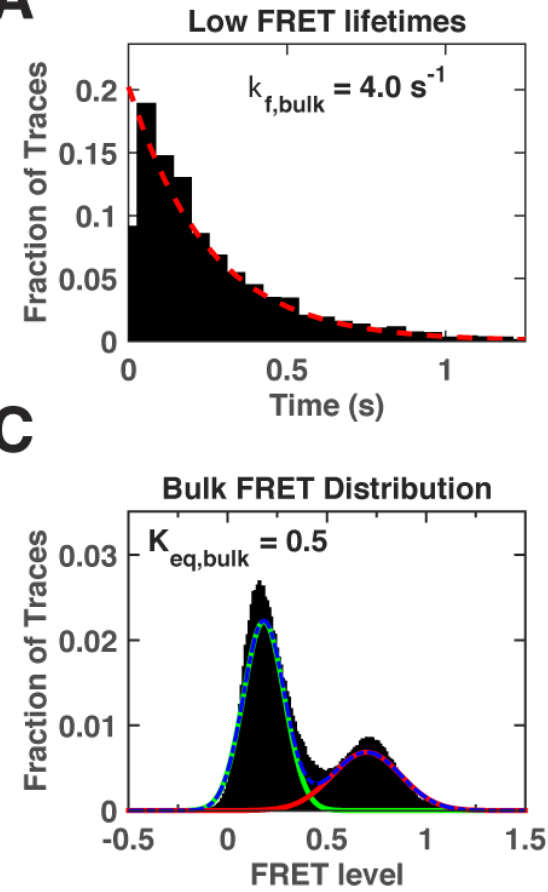
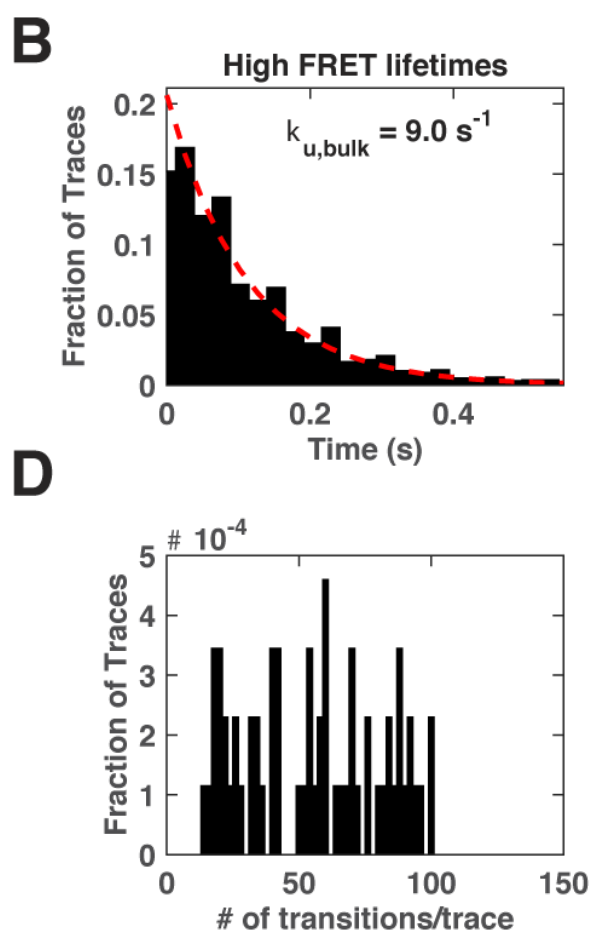

Figure S4-2. smFRET data of P4-P6 variant A225U in 2.4 M NaCl. A) and B) Distribution of the $k_{\mathrm{f}}$ (folding) and $k_{\mathrm{u}}$ (unfolding) rate constants as a function of time. An exponential fit (dashed red lines) to the distribution of lifetimes in the unfolded (A) or folded (B) states of all molecules was used to determine $k_{\mathrm{f}}(\mathrm{A})$ and $k_{\mathrm{u}}(\mathrm{B})$ rate constants (see Experimental Methods in main text). C) Histogram of FRET distribution for all molecules. Distribution is fit to a two-Gaussian model (blue). The equilibrium constant $\left(K_{\text {eq,bulk }}\right)$ was determined from the ratio of the fraction of molecules that are in the high FRET peak (red) versus low FRET peak (green). D) Distribution of the number of transitions per trace. 

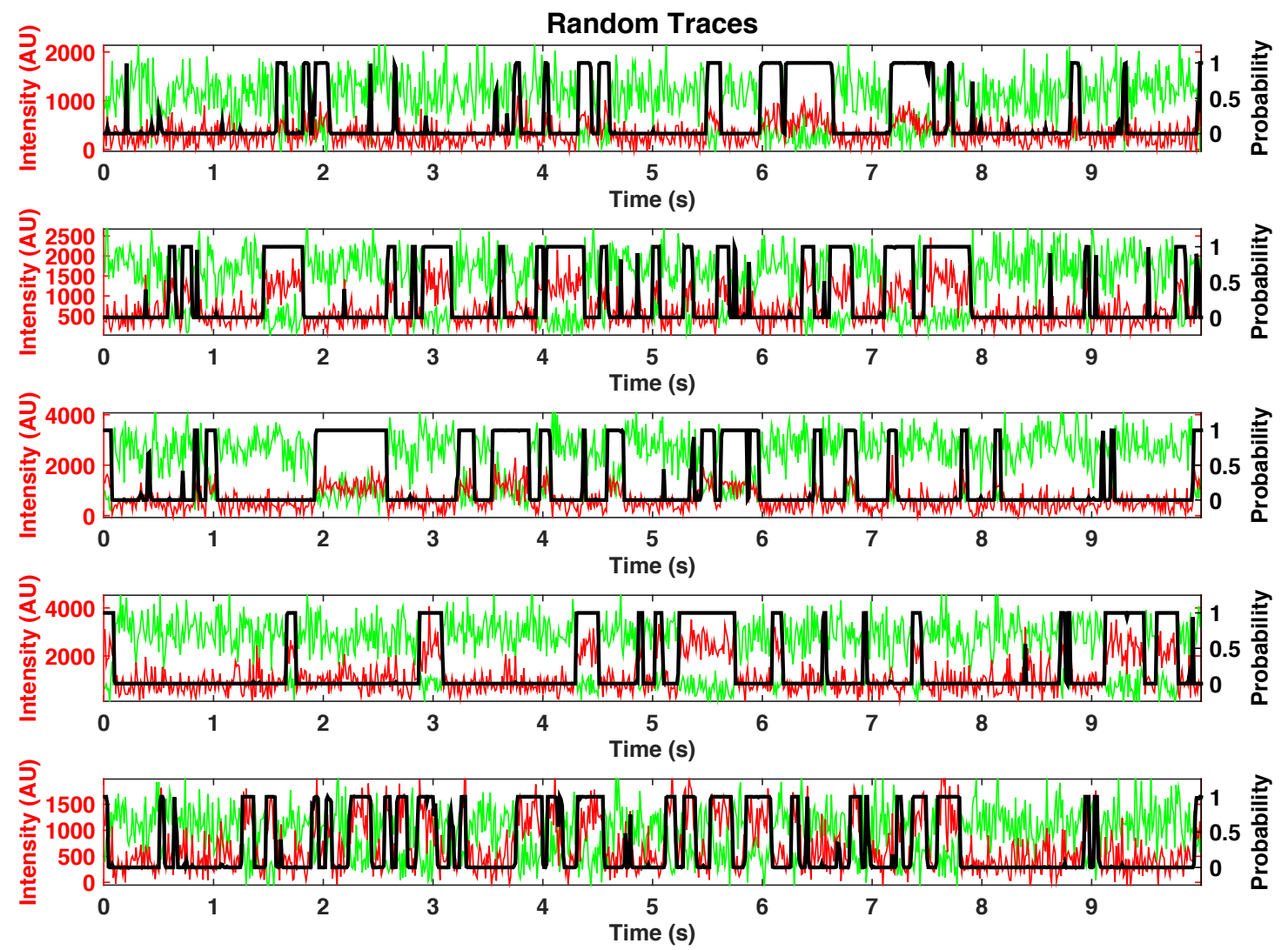

Figure S4-3. Randomly selected FRET traces of P4-P6 variant A225U in 2.4 M NaCl. The intensities of the donor dye (green) and the acceptor dye (red) are shown. The black line denotes the probability of the high FRET state determined by a hidden Markov model fit to the data. ${ }^{6}$ To scale the length of the randomly selected FRET traces and ease their comparison, traces were truncated at the length of the shortest selected trace. 
A
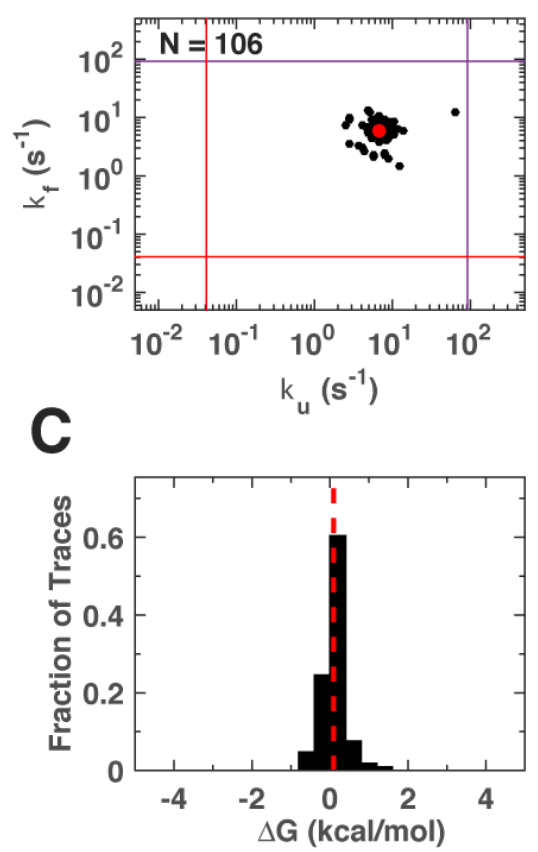

$\mathbf{E}$

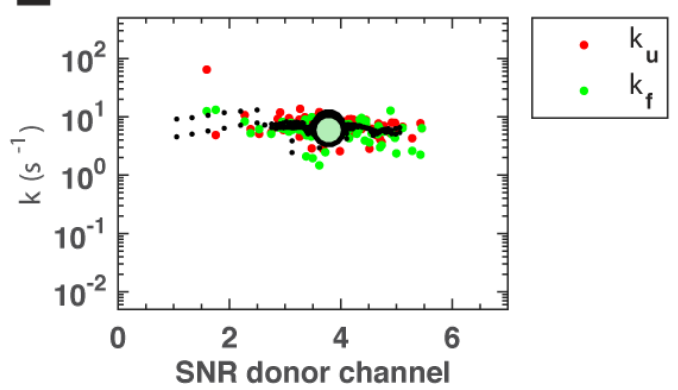

B

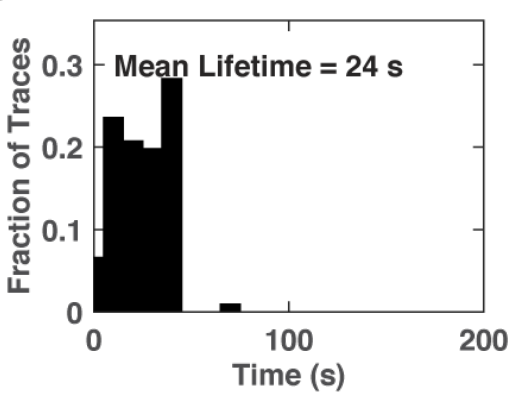

D

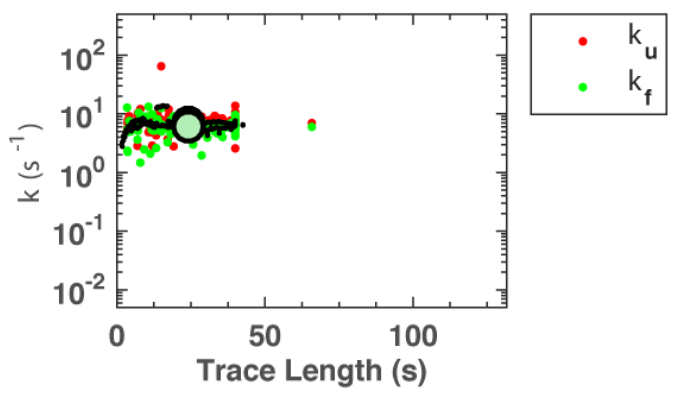

$\mathbf{F}$

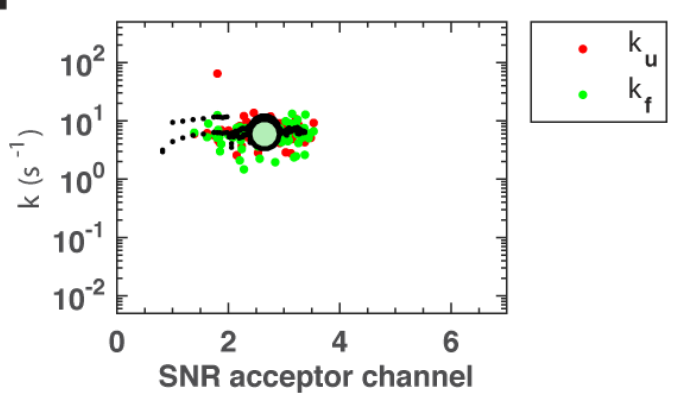

Figure S5-1. smFRET data for P4-P6 A225U in 2.9 M NaCl. A) Scatter plot of fitted values of $k_{\mathrm{f}}$ versus $k_{\mathrm{u}}$ for each molecule. ${ }^{6}$ Purple lines indicate the camera frame rate and red lines indicate the average lifetime of the molecules. The red dot indicates mean folding and unfolding rate constants. TIRF measurements were taken at 91 frames per second (see Experimental Methods in main text). $\mathrm{N}=$ number of molecules. B) Histogram of trace lengths for individual molecules. C) Histogram of $\Delta \mathrm{G}$ values for individual molecules (determined from equilibrium $K_{e q}=k_{f} / k_{u}$ ). The mean value is denoted by the dashed red line. D) Scatter plot of rate constants versus trace length; $k_{\mathrm{f}}$ (green) and $k_{\mathrm{u}}$ (red). The mean value for each rate constant is presented by larger light green $\left(k_{\mathrm{f}}\right)$ and red $\left(k_{\mathrm{u}}\right)$ circles. The black dots are the average of ten values and are shown to provide an empirical guide. E) and F) Rate constants as a function of signal-to-noise ratio (SNR) in the donor channel and acceptor channels respectively. Colors as in (D). 
A
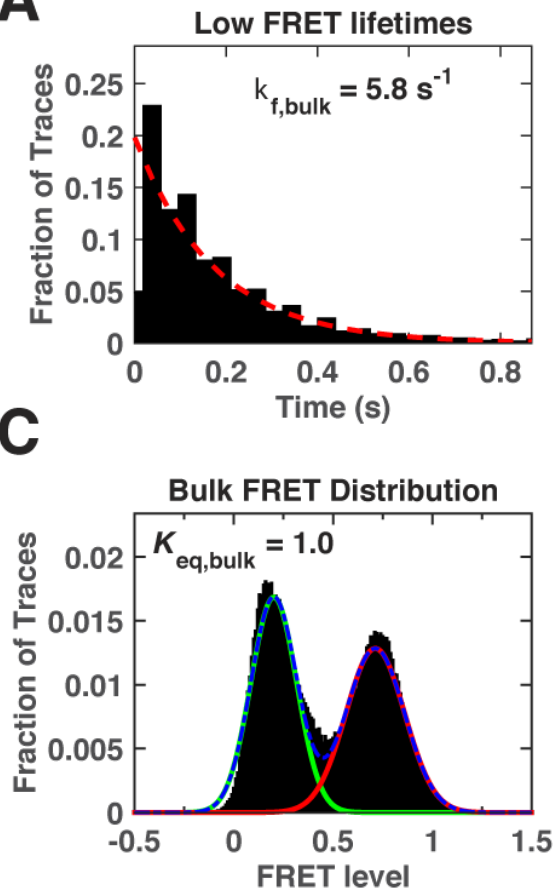
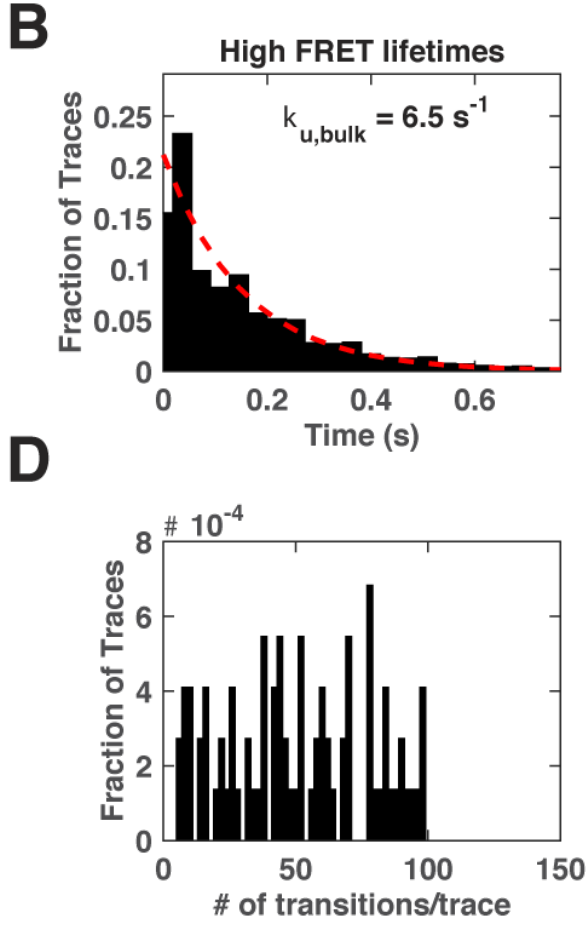

Figure S5-2. smFRET data of P4-P6 variant A225U in 2.9 M NaCl. A) and B) Distribution of the $k_{\mathrm{f}}$ (folding) and $k_{\mathrm{u}}$ (unfolding) rate constants as a function of time. An exponential fit (dashed red lines) to the distribution of lifetimes in the unfolded (A) or folded (B) states of all molecules was used to determine $k_{\mathrm{f}}(\mathrm{A})$ and $k_{\mathrm{u}}(\mathrm{B})$ rate constants (see Experimental Methods in main text). C) Histogram of FRET distribution for all molecules. Distribution is fit to a two-Gaussian model (blue). The equilibrium constant $\left(K_{\text {eq,bulk }}\right)$ was determined from the ratio of the fraction of molecules that are in the high FRET peak (red) versus low FRET peak (green). D) Distribution of the number of transitions per trace. 

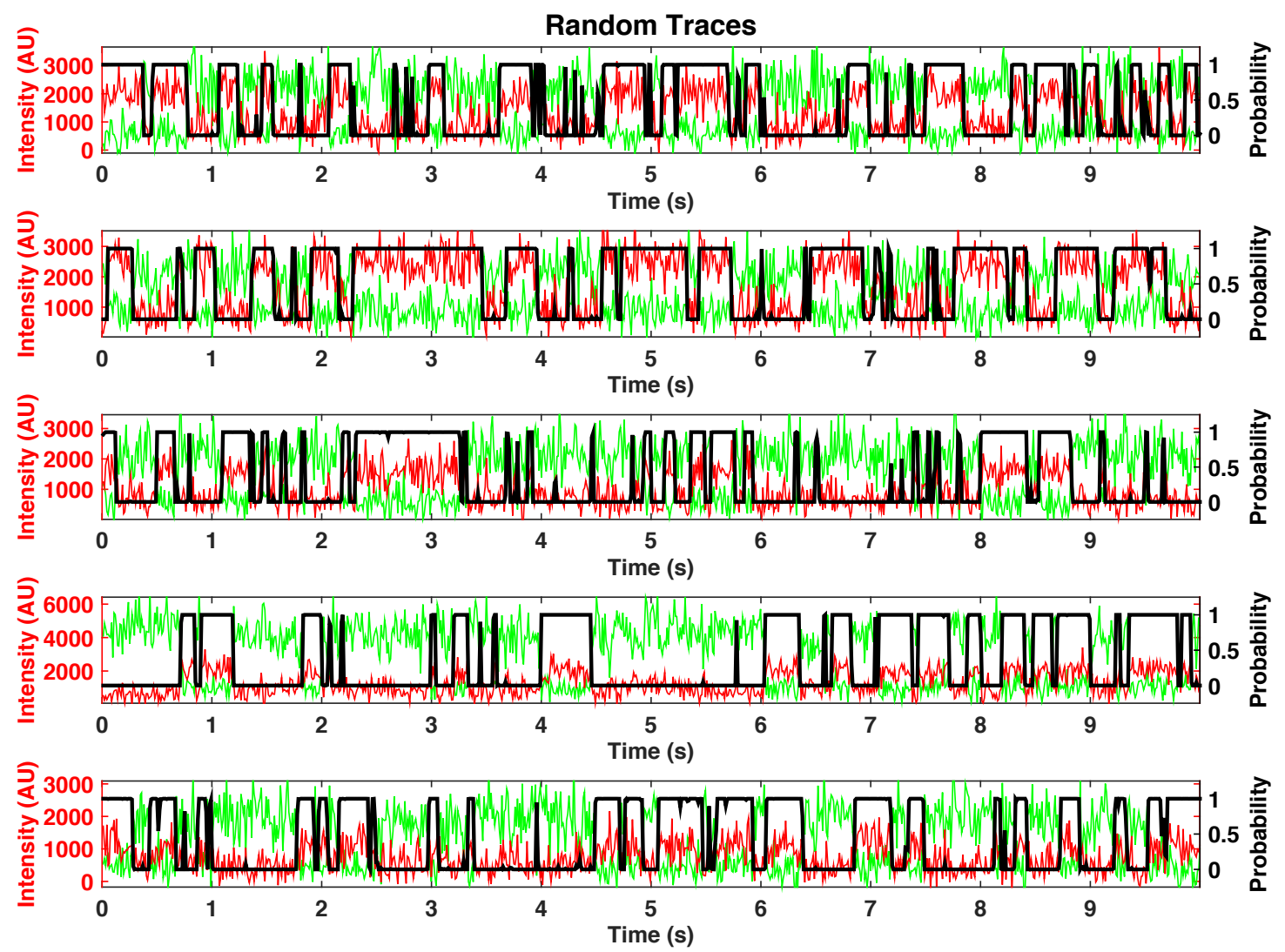

Figure S5-3. Randomly selected FRET traces of P4-P6 variant A225U in 2.9 M NaCl. The intensities of the donor dye (green) and the acceptor dye (red) are shown. The black line denotes the probability of the high FRET state determined by a hidden Markov model fit to the data. ${ }^{6}$ To scale the length of the randomly selected FRET traces and ease their comparison, traces were truncated at the length of the shortest selected trace. 
A

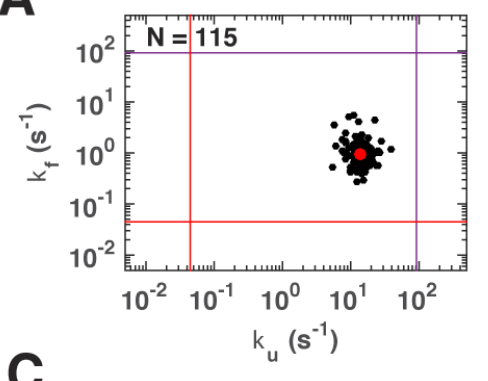

C

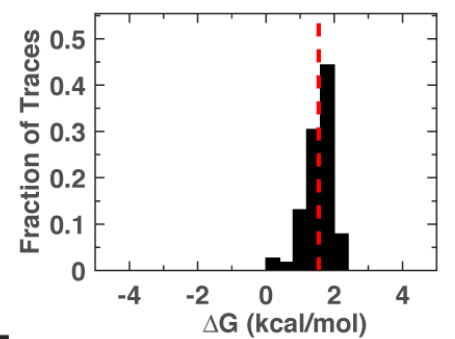

E

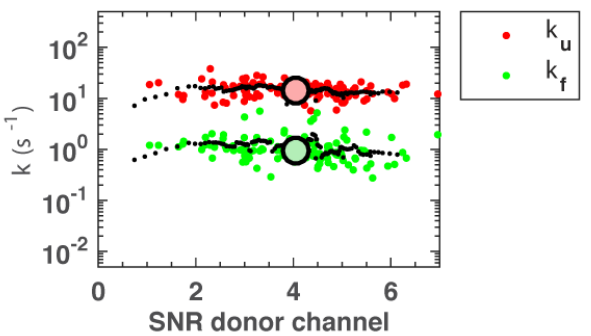

B

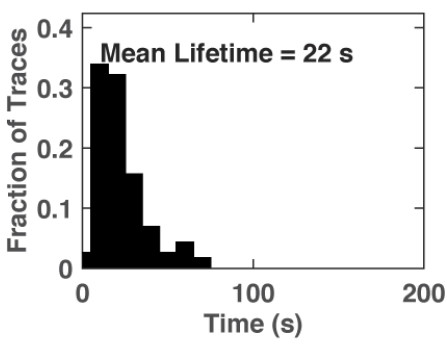

D
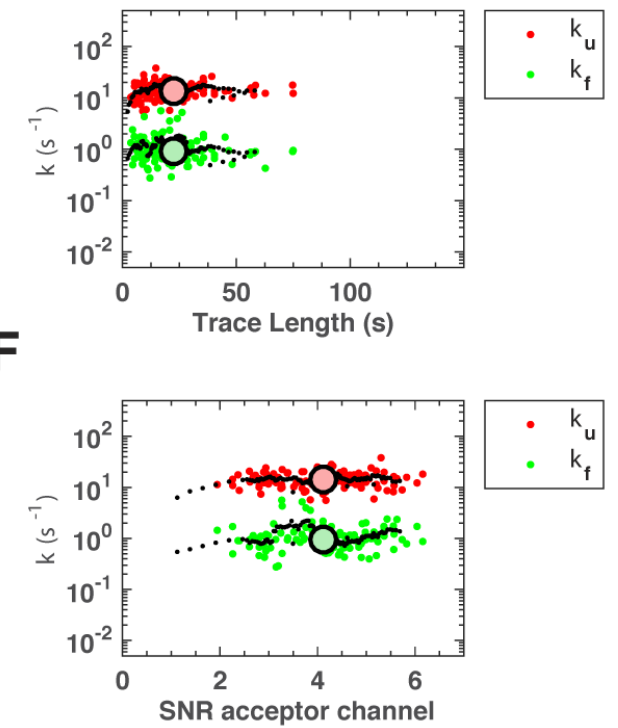

Figure S6-1. smFRET data for P4-P6 A225U in 1.6 M KCl. A) Scatter plot of fitted values of $k_{\mathrm{f}}$ versus $k_{\mathrm{u}}$ for each molecule. ${ }^{6}$ Purple lines indicate the camera frame rate and red lines indicate the average lifetime of the molecules. The red dot indicates mean folding and unfolding rate constants. TIRF measurements were taken at 91 frames per second (see Experimental Methods in main text). $\mathrm{N}=$ number of molecules. B) Histogram of trace lengths for individual molecules. C) Histogram of $\Delta G$ values for individual molecules (determined from equilibrium $K_{e q}=k_{f} / k_{u}$ ). The mean value is denoted by the dashed red line. D) Scatter plot of rate constants versus trace length; $k_{\mathrm{f}}$ (green) and $k_{\mathrm{u}}(\mathrm{red})$. The mean value for each rate constant is presented by larger light green $\left(k_{\mathrm{f}}\right)$ and red $\left(k_{\mathrm{u}}\right)$ circles. The black dots are the average of ten values and are shown to provide an empirical guide. E) and F) Rate constants as a function of signalto-noise ratio (SNR) in the donor channel and acceptor channels respectively. Colors as in (D). 
A
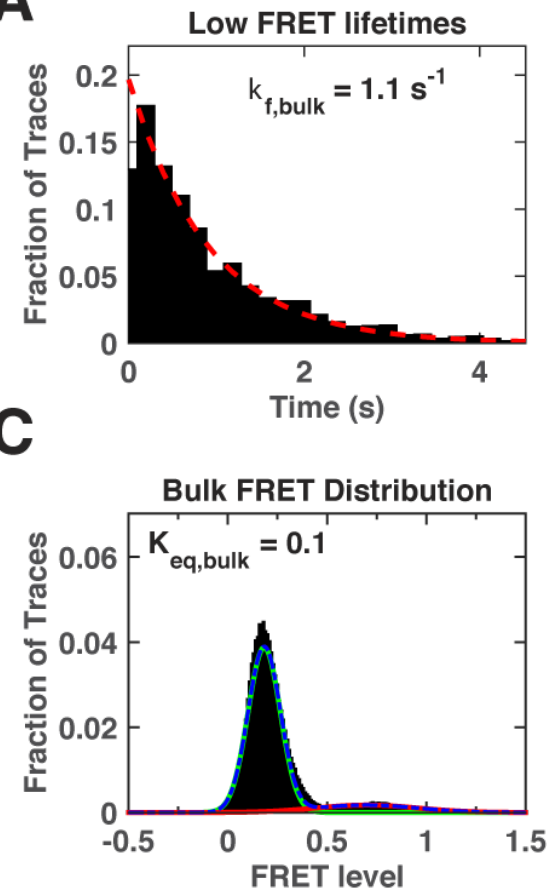
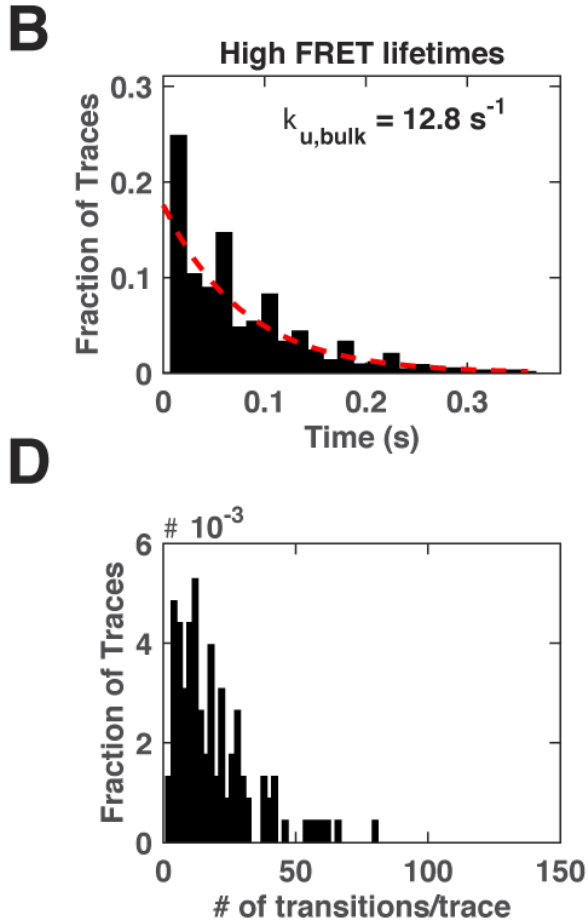

Figure S6-2. smFRET data of P4-P6 variant A225U in 1.6 M KCl. A) and B) Distribution of the $k_{\mathrm{f}}$ (folding) and $k_{\mathrm{u}}$ (unfolding) rate constants as a function of time. An exponential fit (dashed red lines) to the distribution of lifetimes in the unfolded (A) or folded (B) states of all molecules was used to determine $k_{\mathrm{f}}(\mathrm{A})$ and $k_{\mathrm{u}}(\mathrm{B})$ rate constants (see Experimental Methods in main text). C) Histogram of FRET distribution for all molecules. Distribution is fit to a two-Gaussian model (blue). The equilibrium constant $\left(K_{\text {eq,bulk }}\right)$ was determined from the ratio of the fraction of molecules that are in the high FRET peak (red) versus low FRET peak (green). D) Distribution of the number of transitions per trace. 

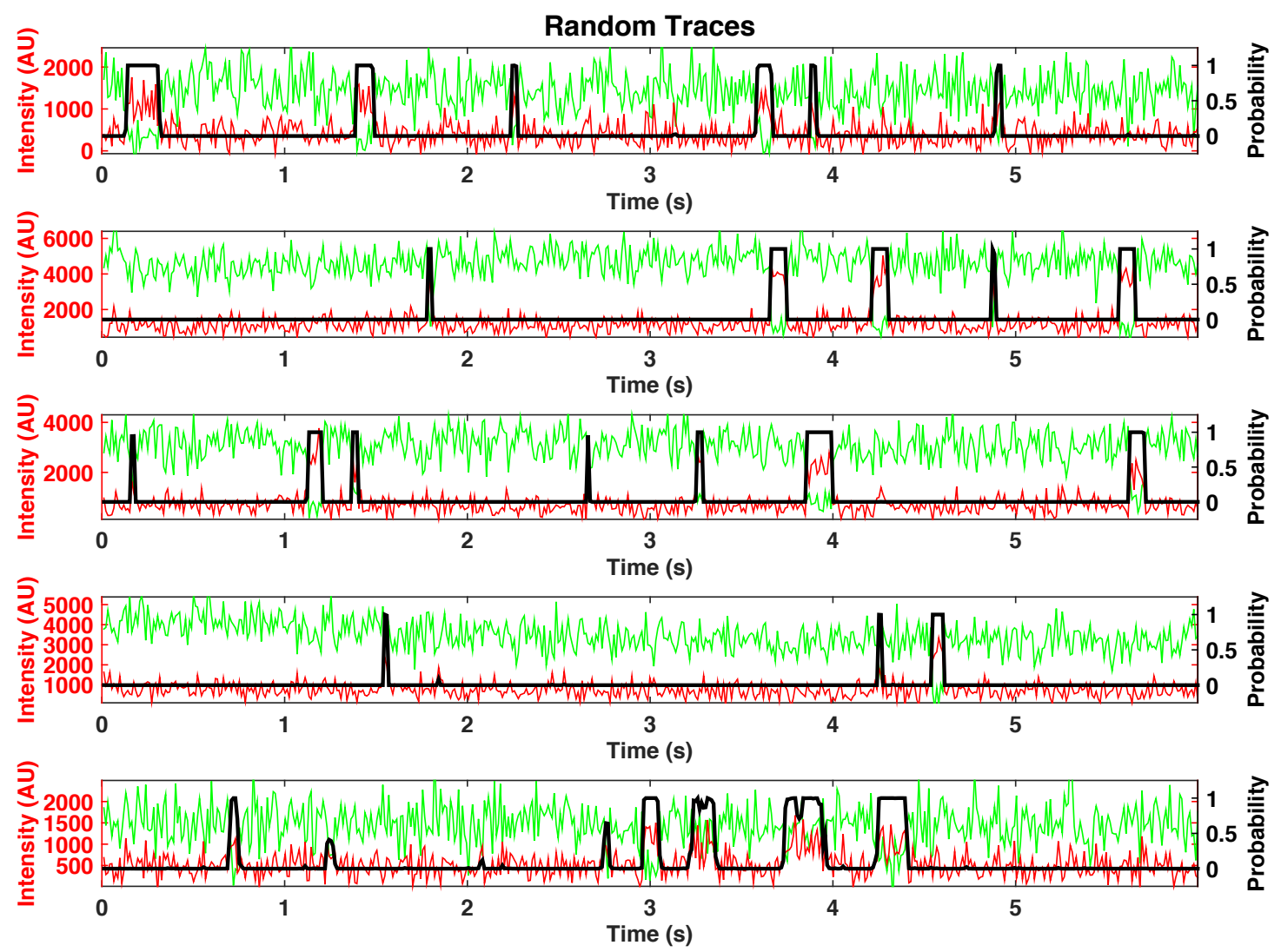

Figure S6-3. Randomly selected FRET traces of P4-P6 variant A225U in 1.6 M KCl. The intensities of the donor dye (green) and the acceptor dye (red) are shown. The black line denotes the probability of the high FRET state determined by a hidden Markov model fit to the data. ${ }^{6}$ To scale the length of the randomly selected FRET traces and ease their comparison, traces were truncated at the length of the shortest selected trace. 

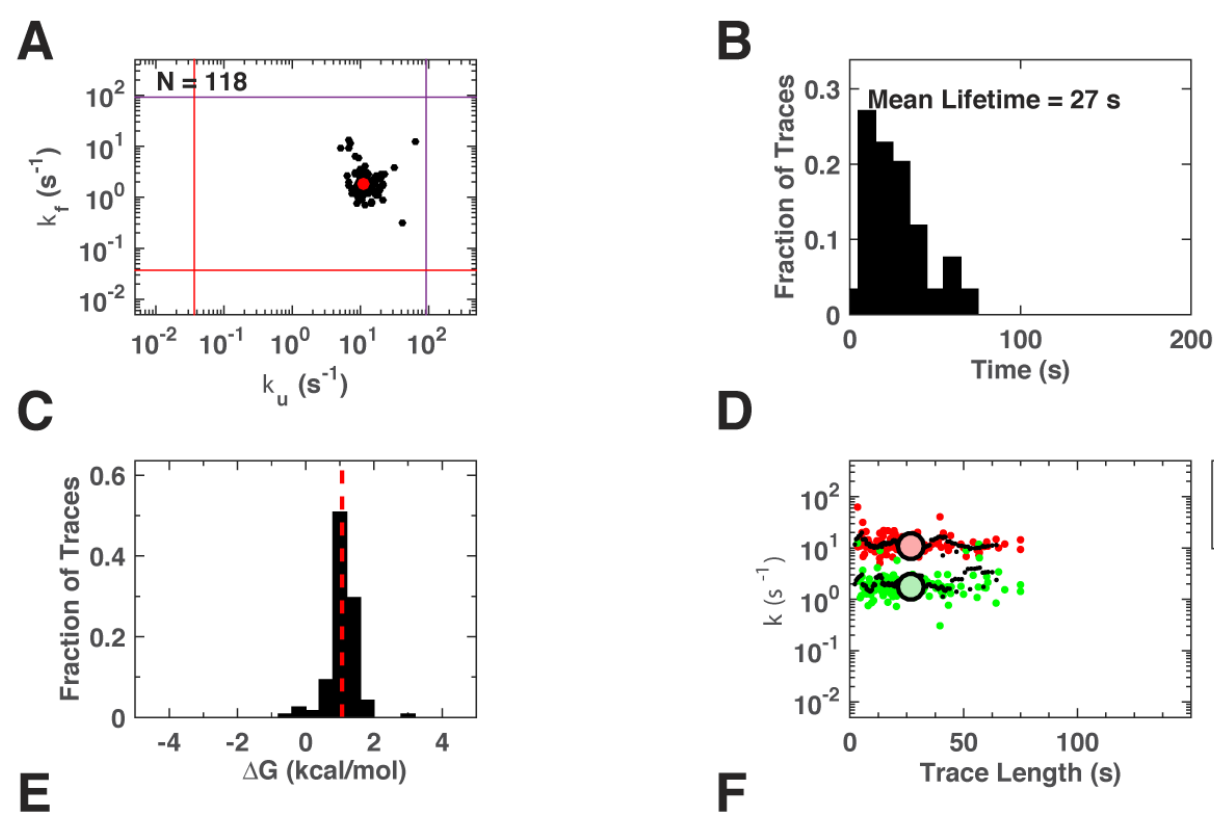

D
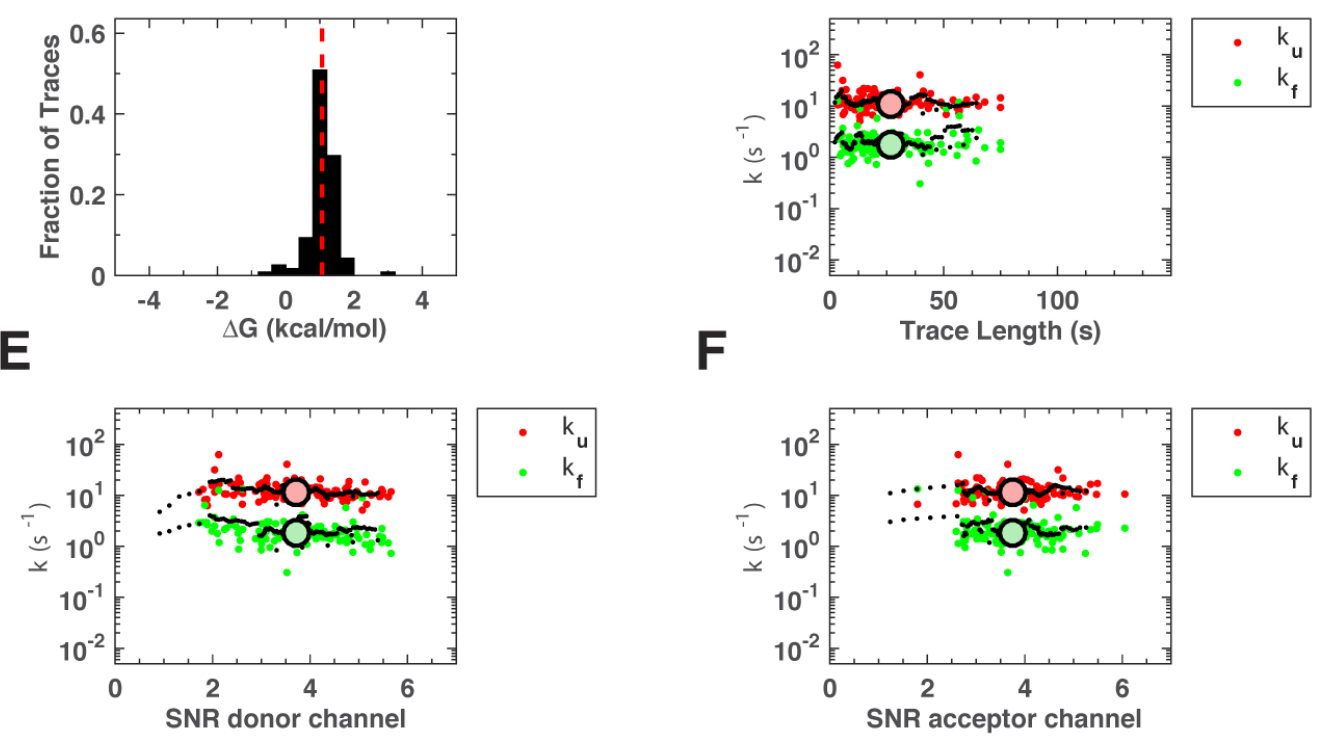

Figure S7-1. smFRET data for P4-P6 A225U in 2.0 M KCl. A) Scatter plot of fitted values of $k_{\mathrm{f}}$ versus $k_{\mathrm{u}}$ for each molecule. ${ }^{6}$ Purple lines indicate the camera frame rate and red lines indicate the average lifetime of the molecules. The red dot indicates mean folding and unfolding rate constants. TIRF measurements were taken at 91 frames per second (see Experimental Methods in main text). $\mathrm{N}=$ number of molecules. B) Histogram of trace lengths for individual molecules. C) Histogram of $\Delta \mathrm{G}$ values for individual molecules (determined from equilibrium $K_{e q}=k_{f} / k_{u}$ ). The mean value is denoted by the dashed red line. D) Scatter plot of rate constants versus trace length; $k_{\mathrm{f}}$ (green) and $k_{\mathrm{u}}$ (red). The mean value for each rate constant is presented by larger light green $\left(k_{\mathrm{f}}\right)$ and red $\left(k_{\mathrm{u}}\right)$ circles. The black dots are the average of ten values and are shown to provide an empirical guide. E) and F) Rate constants as a function of signalto-noise ratio (SNR) in the donor channel and acceptor channels respectively. Colors as in (D). 
A
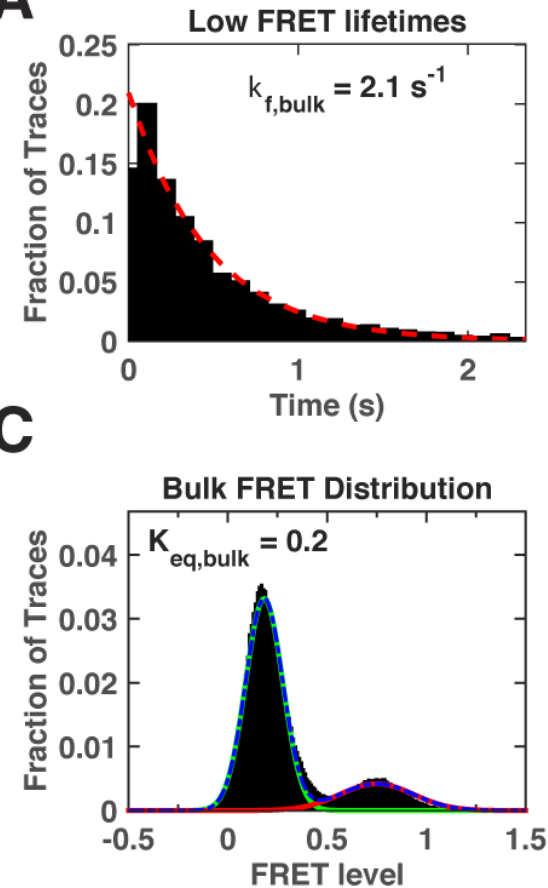
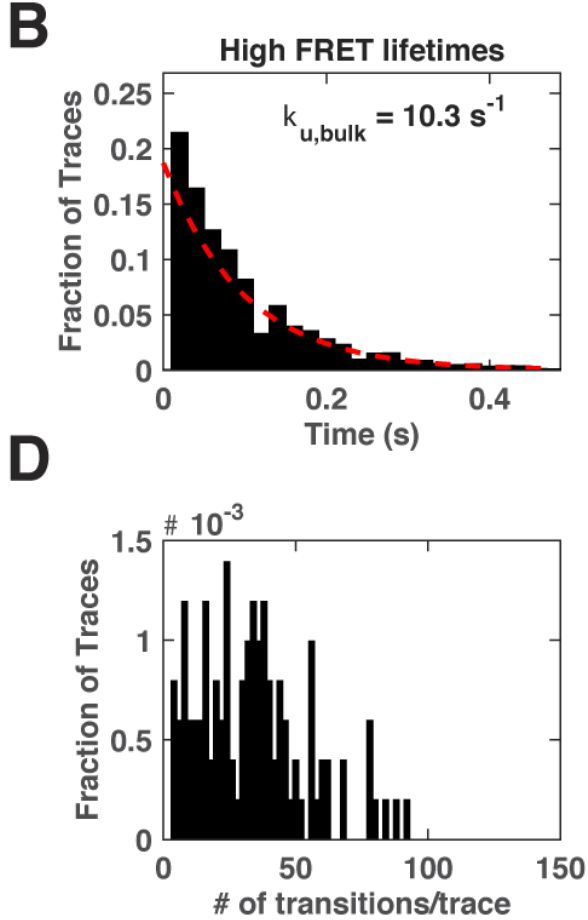

Figure S7-2. smFRET data of P4-P6 variant A225U in 2.0 M KCl. A) and B) Distribution of the $k_{\mathrm{f}}$ (folding) and $k_{\mathrm{u}}$ (unfolding) rate constants as a function of time. An exponential fit (dashed red lines) to the distribution of lifetimes in the unfolded (A) or folded (B) states of all molecules was used to determine $k_{\mathrm{f}}(\mathrm{A})$ and $k_{\mathrm{u}}(\mathrm{B})$ rate constants (see Experimental Methods in main text). C) Histogram of FRET distribution for all molecules. Distribution is fit to a two-Gaussian model (blue). The equilibrium constant $\left(K_{\text {eq,bulk }}\right)$ was determined from the ratio of the fraction of molecules that are in the high FRET peak (red) versus low FRET peak (green). D) Distribution of the number of transitions per trace. 

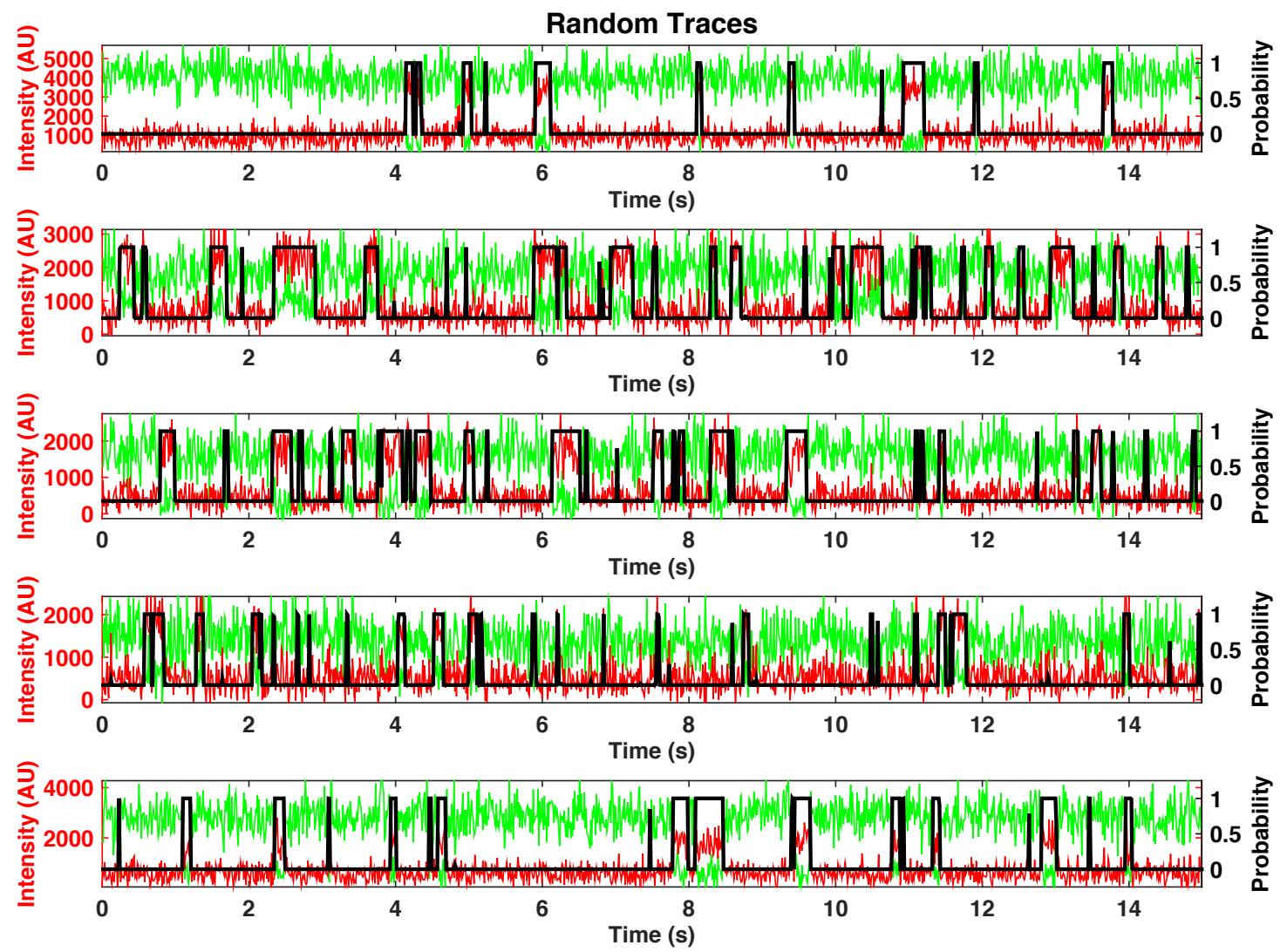

Figure S7-3. Randomly selected FRET traces of P4-P6 variant A225U in 2.0 M KCl. The intensities of the donor dye (green) and the acceptor dye (red) are shown. The black line denotes the probability of the high FRET state determined by a hidden Markov model fit to the data. ${ }^{6}$ To scale the length of the randomly selected FRET traces and ease their comparison, traces were truncated at the length of the shortest selected trace. 
A

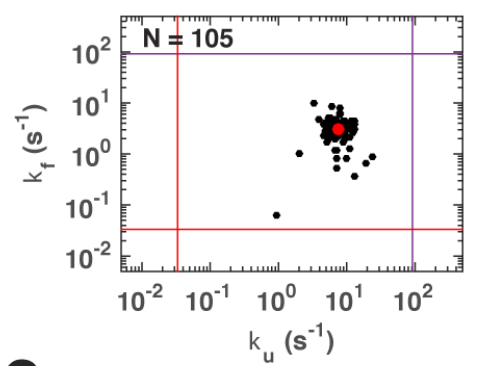

C

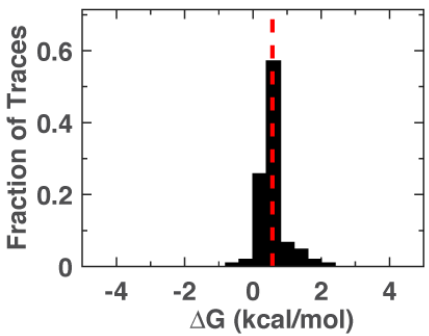

$\mathbf{E}$

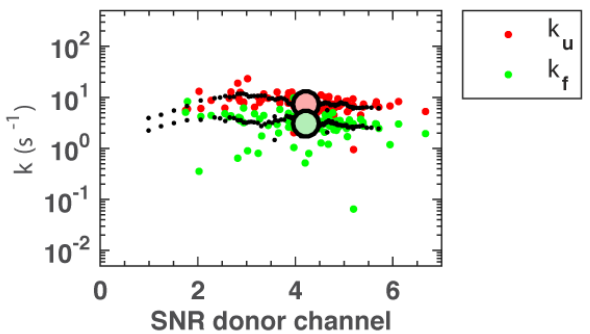

B

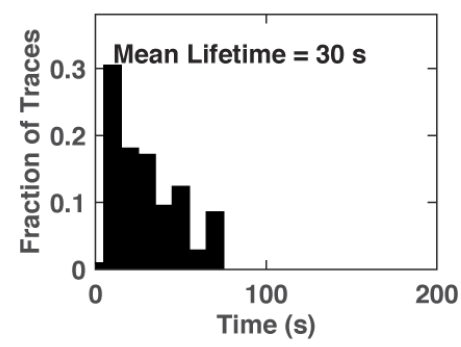

D

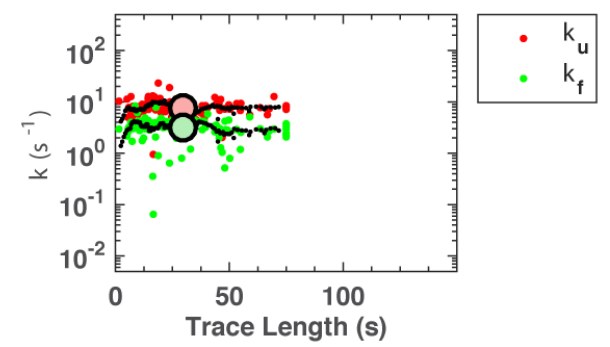

$\mathbf{F}$

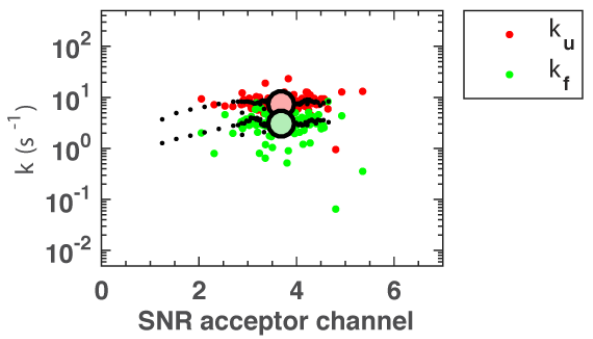

Figure S8-1. smFRET data for P4-P6 A225U in 2.5 M KCl. A) Scatter plot of fitted values of $k_{\mathrm{f}}$ versus $k_{\mathrm{u}}$ for each molecule. ${ }^{6}$ Purple lines indicate the camera frame rate and red lines indicate the average lifetime of the molecules. The red dot indicates mean folding and unfolding rate constants. TIRF measurements were taken at 91 frames per second (see Experimental Methods in main text). $\mathrm{N}=$ number of molecules. B) Histogram of trace lengths for individual molecules. C) Histogram of $\Delta G$ values for individual molecules (determined from equilibrium $K_{e q}=k_{f} / k_{u}$ ). The mean value is denoted by the dashed red line. D) Scatter plot of rate constants versus trace length; $k_{\mathrm{f}}$ (green) and $k_{\mathrm{u}}(\mathrm{red})$. The mean value for each rate constant is presented by larger light green $\left(k_{\mathrm{f}}\right)$ and red $\left(k_{\mathrm{u}}\right)$ circles. The black dots are the average of ten values and are shown to provide an empirical guide. E) and F) Rate constants as a function of signalto-noise ratio (SNR) in the donor channel and acceptor channels respectively. Colors as in (D). 
A
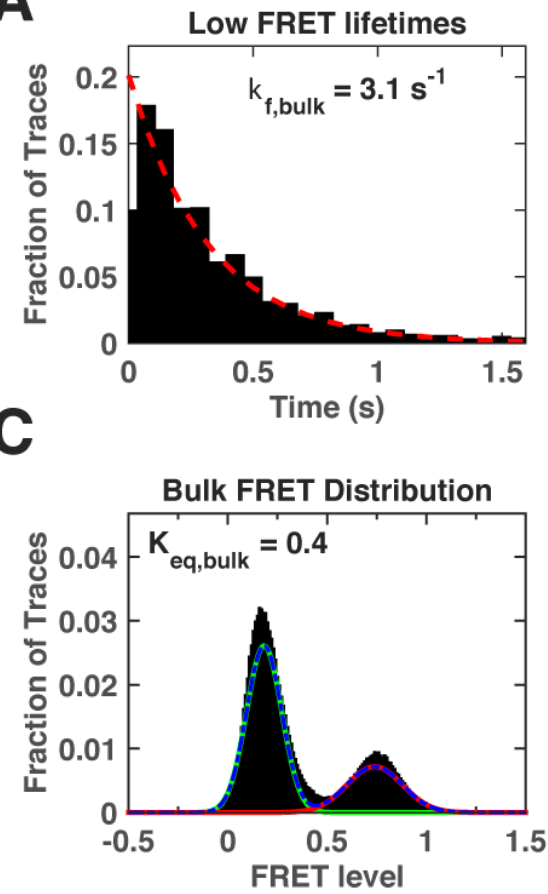
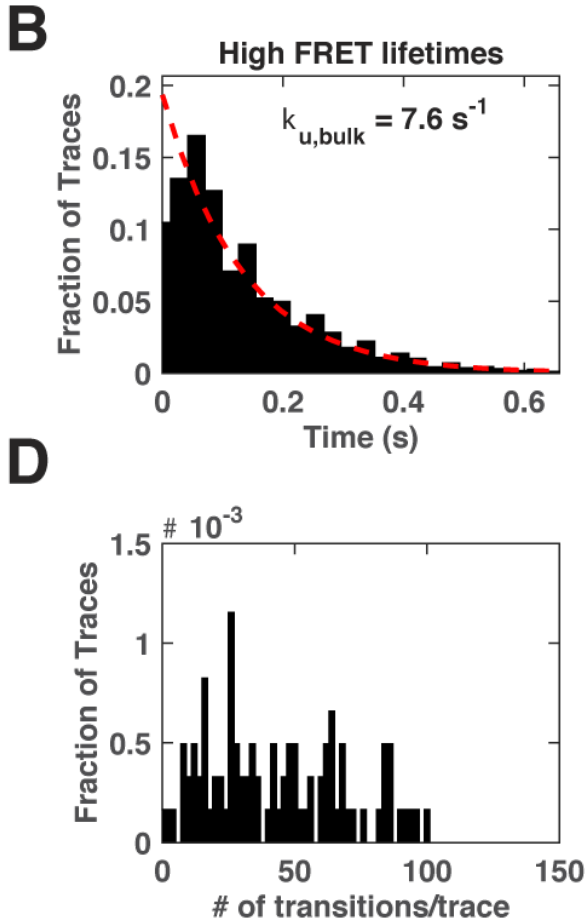

Figure S8-2. smFRET data of P4-P6 variant A225U in 2.5 M KCl. A) and B) Distribution of the $k_{\mathrm{f}}$ (folding) and $k_{\mathrm{u}}$ (unfolding) rate constants as a function of time. An exponential fit (dashed red lines) to the distribution of lifetimes in the unfolded (A) or folded (B) states of all molecules was used to determine $k_{\mathrm{f}}(\mathrm{A})$ and $k_{\mathrm{u}}(\mathrm{B})$ rate constants (see Experimental Methods in main text). C) Histogram of FRET distribution for all molecules. Distribution is fit to a two-Gaussian model (blue). The equilibrium constant $\left(K_{\text {eq,bulk }}\right)$ was determined from the ratio of the fraction of molecules that are in the high FRET peak (red) versus low FRET peak (green). D) Distribution of the number of transitions per trace. 

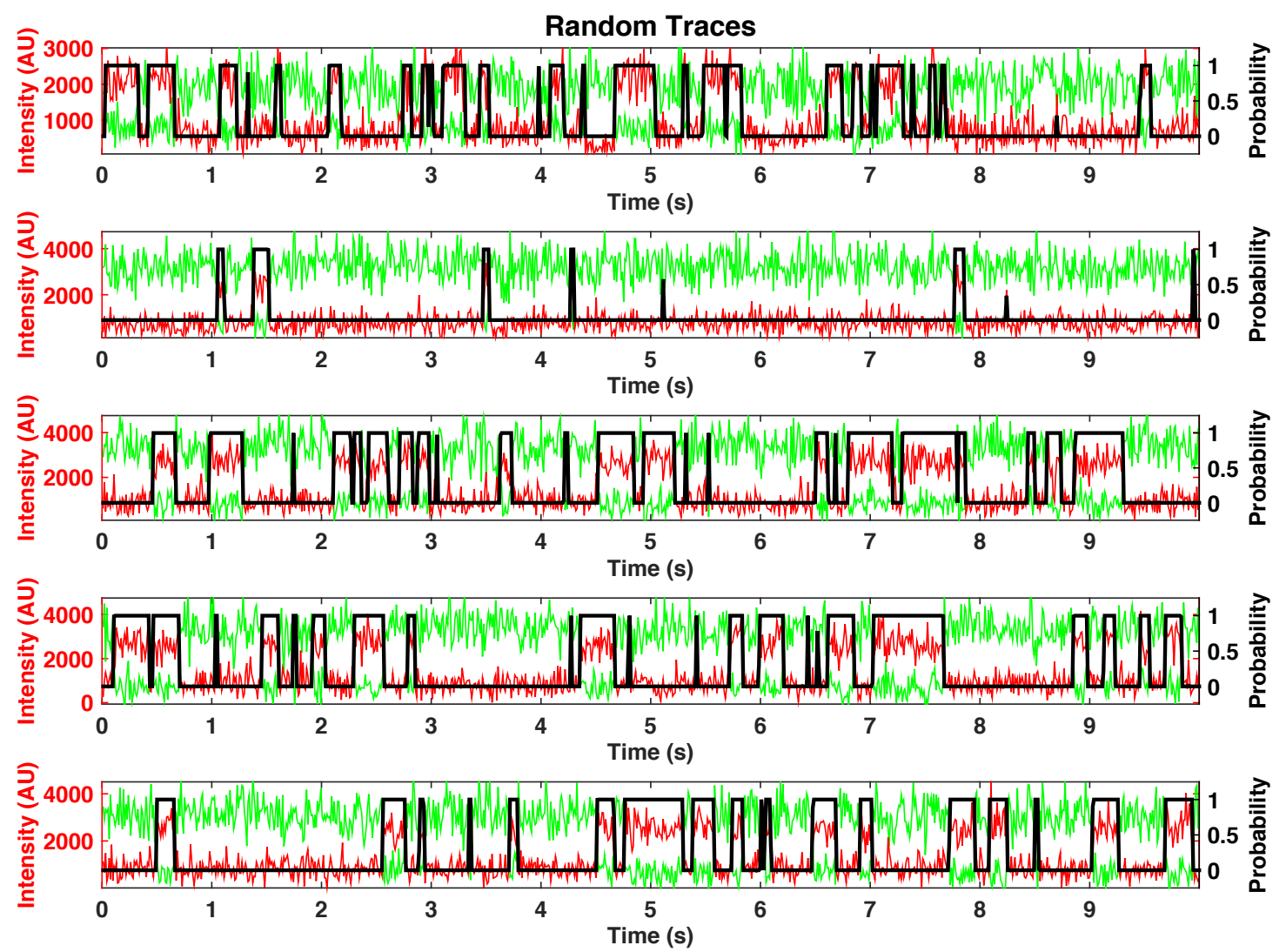

Figure S8-3. Randomly selected FRET traces of P4-P6 variant A225U in 2.5 M KCl. The intensities of the donor dye (green) and the acceptor dye (red) are shown. The black line denotes the probability of the high FRET state determined by a hidden Markov model fit to the data. ${ }^{6}$ To scale the length of the randomly selected FRET traces and ease their comparison, traces were truncated at the length of the shortest selected trace. 
A
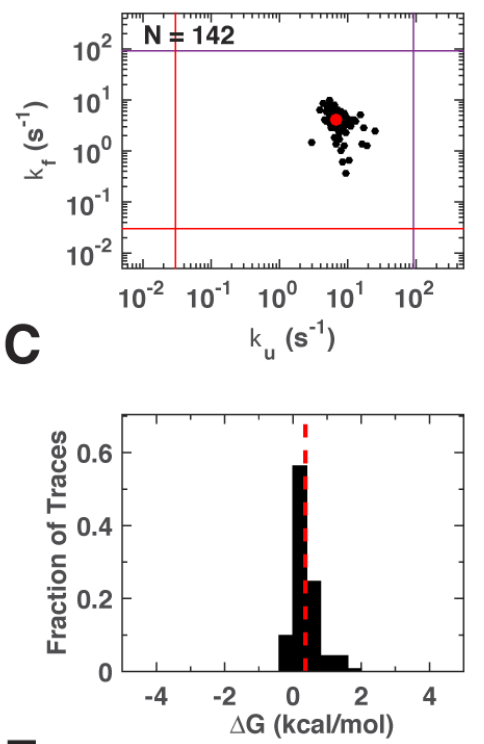

$\mathbf{E}$

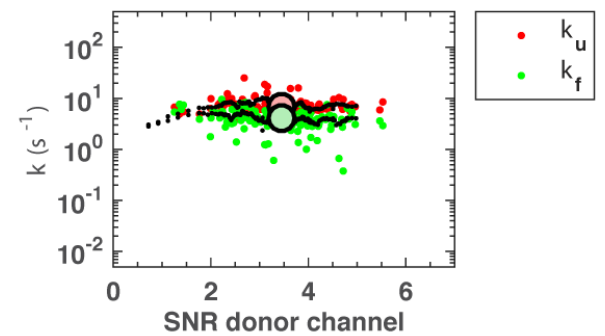

B
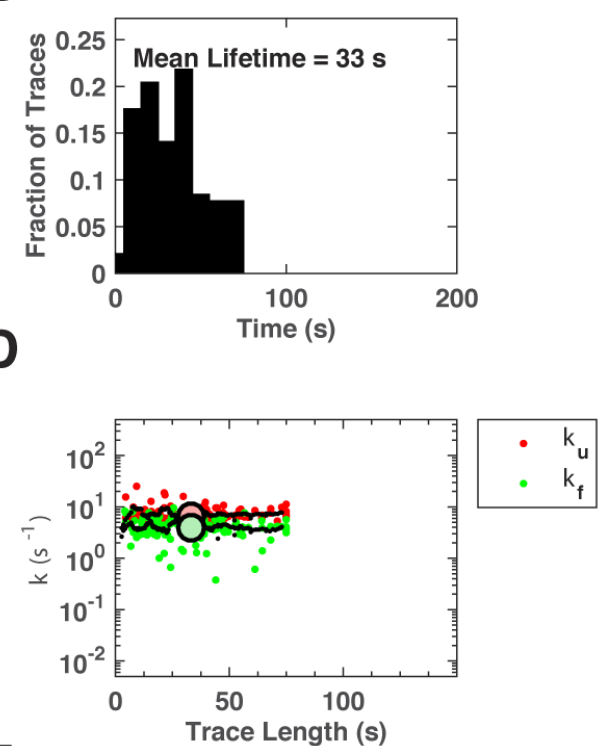

$\mathbf{F}$

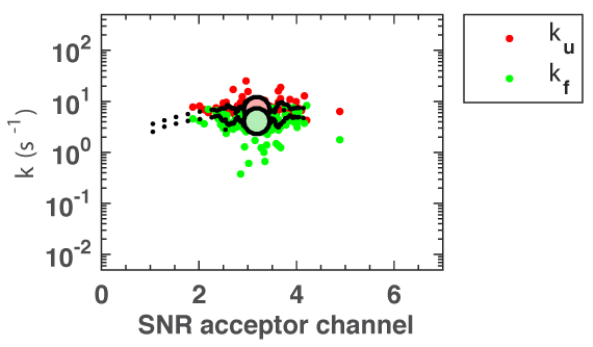

Figure S9-1. smFRET data for P4-P6 A225U in 2.8 M KCl. A) Scatter plot of fitted values of $k_{\mathrm{f}}$ versus $k_{\mathrm{u}}$ for each molecule. ${ }^{6}$ Purple lines indicate the camera frame rate and red lines indicate the average lifetime of the molecules. The red dot indicates mean folding and unfolding rate constants. TIRF measurements were taken at 91 frames per second (see Experimental Methods in main text). $\mathrm{N}$ = number of molecules. B) Histogram of trace lengths for individual molecules. C) Histogram of $\Delta G$ values for individual molecules (determined from equilibrium $K_{e q}=k_{f} / k_{u}$ ). The mean value is denoted by the dashed red line. D) Scatter plot of rate constants versus trace length; $k_{\mathrm{f}}$ (green) and $k_{\mathrm{u}}(\mathrm{red})$. The mean value for each rate constant is presented by larger light green $\left(k_{\mathrm{f}}\right)$ and red $\left(k_{\mathrm{u}}\right)$ circles. The black dots are the average of ten values and are shown to provide an empirical guide. E) and F) Rate constants as a function of signalto-noise ratio (SNR) in the donor channel and acceptor channels respectively. Colors as in (D). 
A
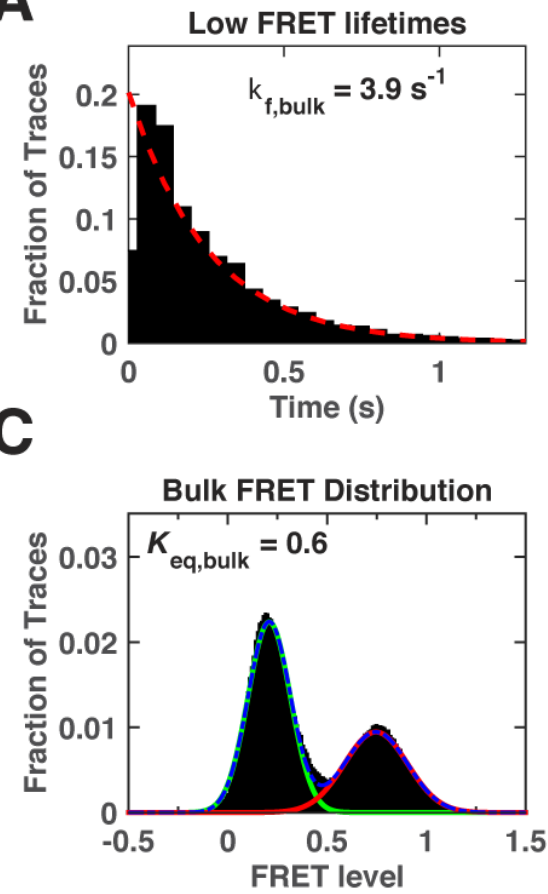
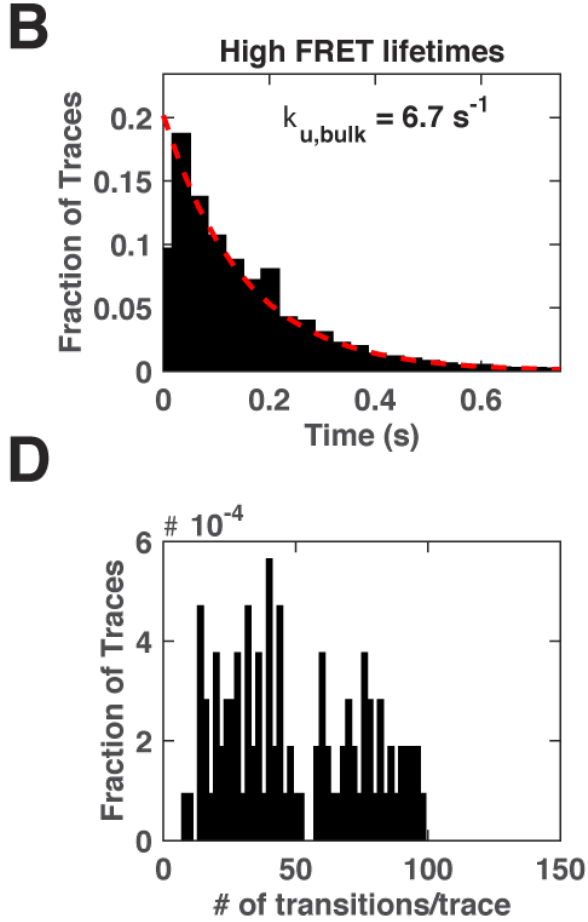

Figure S9-2. smFRET data of P4-P6 variant A225U in $2.8 \mathbf{~ M ~ K C l}$. A) and B) Distribution of the $k_{\mathrm{f}}$ (folding) and $k_{\mathrm{u}}$ (unfolding) rate constants as a function of time. An exponential fit (dashed red lines) to the distribution of lifetimes in the unfolded (A) or folded (B) states of all molecules was used to determine $k_{\mathrm{f}}(\mathrm{A})$ and $k_{\mathrm{u}}(\mathrm{B})$ rate constants (see Experimental Methods in main text). C) Histogram of FRET distribution for all molecules. Distribution is fit to a two-Gaussian model (blue). The equilibrium constant $\left(K_{\text {eq,bulk }}\right)$ was determined from the ratio of the fraction of molecules that are in the high FRET peak (red) versus low FRET peak (green). D) Distribution of the number of transitions per trace. 

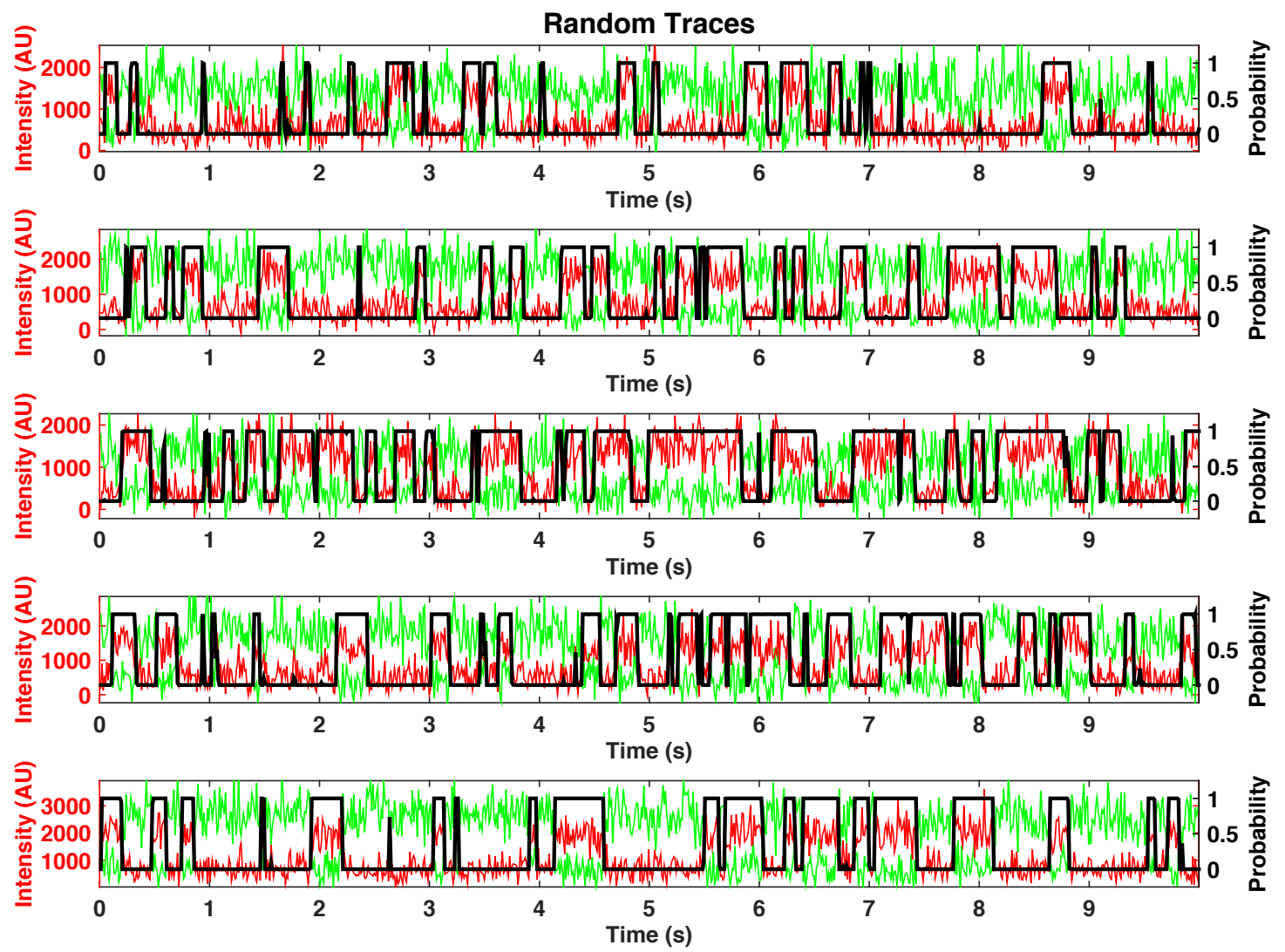

Figure S9-3. Randomly selected FRET traces of P4-P6 variant A225U in 2.8 M KCl. The intensities of the donor dye (green) and the acceptor dye (red) are shown. The black line denotes the probability of the high FRET state determined by a hidden Markov model fit to the data. ${ }^{6}$ To scale the length of the randomly selected FRET traces and ease their comparison, traces were truncated at the length of the shortest selected trace. 
A

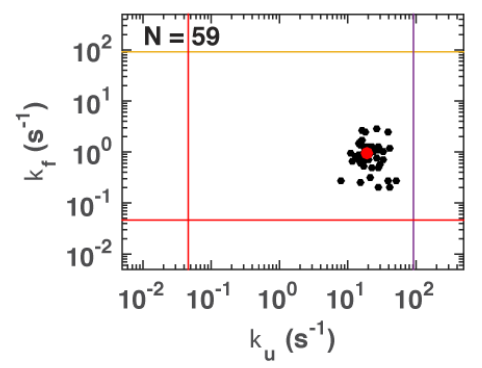

C

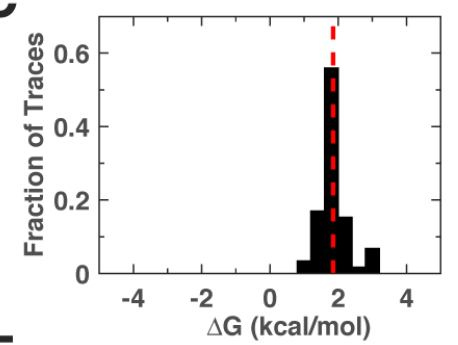

$\mathbf{E}$

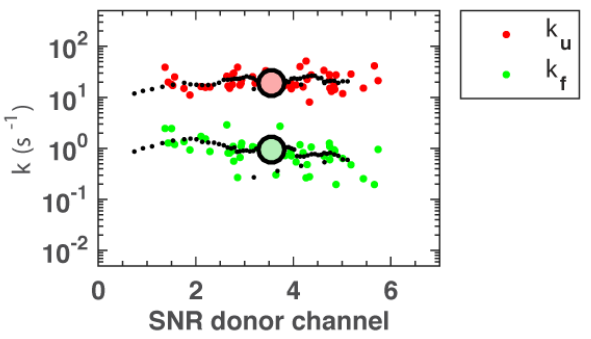

B

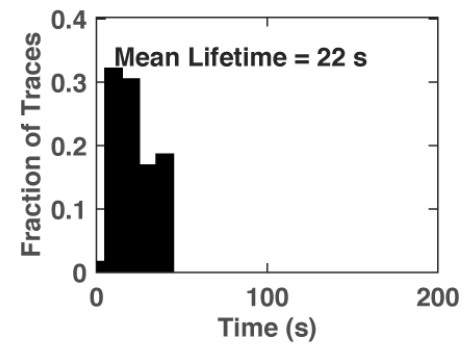

D

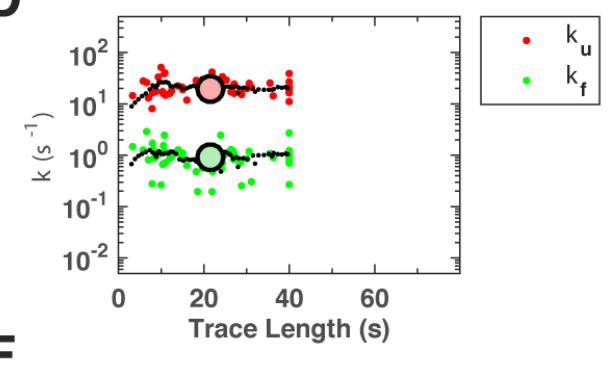

$\mathbf{F}$

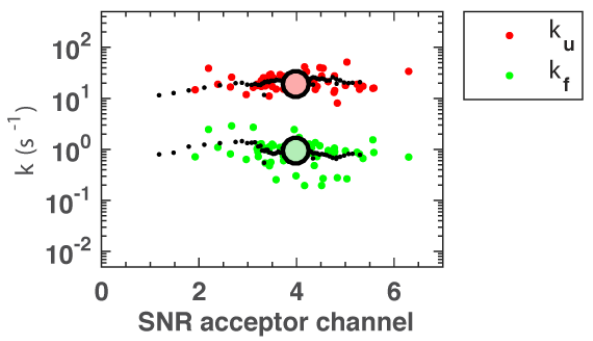

Figure S10-1. smFRET data for P4-P6 A225U in 2.0 M RbCl. A) Scatter plot of fitted values of $k_{\mathrm{f}}$ versus $k_{\mathrm{u}}$ for each molecule. ${ }^{6}$ Purple lines indicate the camera frame rate and red lines indicate the average lifetime of the molecules. The red dot indicates mean folding and unfolding rate constants. TIRF measurements were taken at 91 frames per second (see Experimental Methods in main text). $\mathrm{N}=$ number of molecules. B) Histogram of trace lengths for individual molecules. C) Histogram of $\Delta \mathrm{G}$ values for individual molecules (determined from equilibrium $K_{e q}=k_{f} / k_{u}$ ). The mean value is denoted by the dashed red line. D) Scatter plot of rate constants versus trace length; $k_{\mathrm{f}}$ (green) and $k_{\mathrm{u}}$ (red). The mean value for each rate constant is presented by larger light green $\left(k_{\mathrm{f}}\right)$ and red $\left(k_{\mathrm{u}}\right)$ circles. The black dots are the average of ten values and are shown to provide an empirical guide. E) and F) Rate constants as a function of signal-to-noise ratio (SNR) in the donor channel and acceptor channels respectively. Colors as in (D). 
A

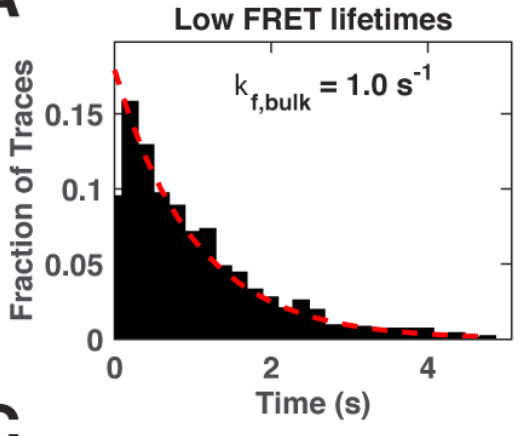

Bulk FRET Distribution

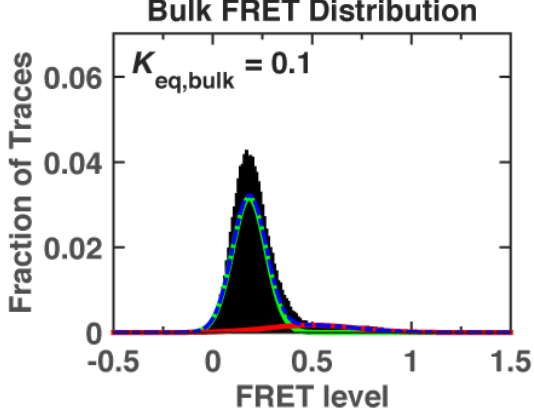

B
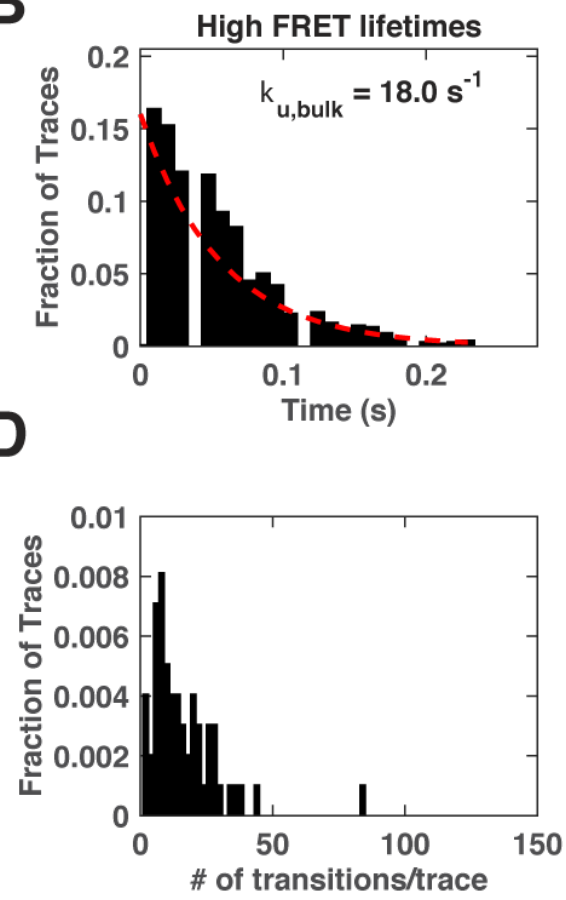

Figure S10-2. smFRET data of P4-P6 variant A225U in 2.0 M RbCl. A) and B) Distribution of the $k_{\mathrm{f}}$ (folding) and $k_{\mathrm{u}}$ (unfolding) rate constants as a function of time. An exponential fit (dashed red lines) to the distribution of lifetimes in the unfolded (A) or folded (B) states of all molecules was used to determine $k_{\mathrm{f}}(\mathrm{A})$ and $k_{\mathrm{u}}(\mathrm{B})$ rate constants (see Experimental Methods in main text). C) Histogram of FRET distribution for all molecules. Distribution is fit to a two-Gaussian model (blue). The equilibrium constant $\left(K_{\text {eq,bulk }}\right)$ was determined from the ratio of the fraction of molecules that are in the high FRET peak (red) versus low FRET peak (green). D) Distribution of the number of transitions per trace. 

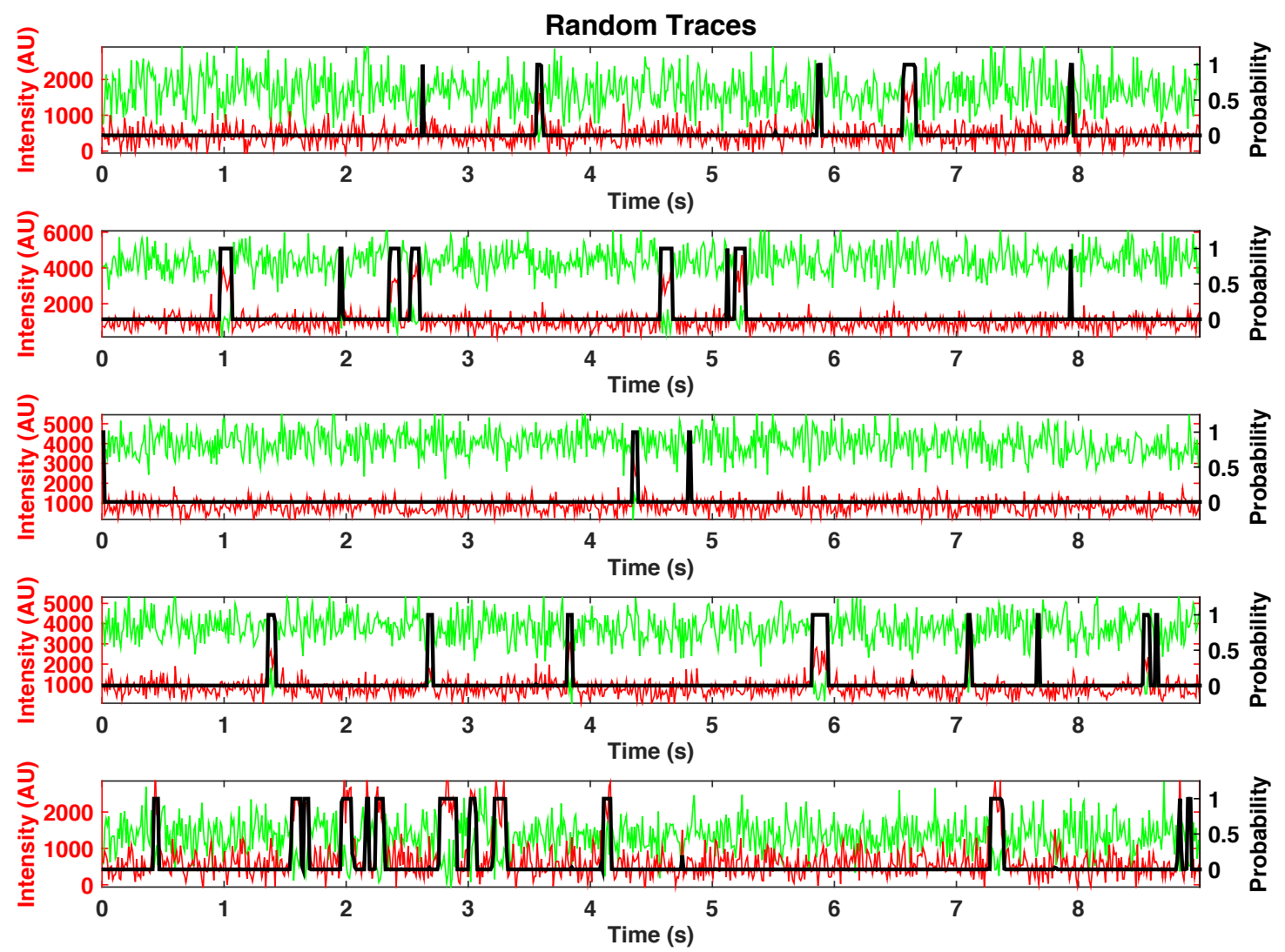

Figure S10-3. Randomly selected FRET traces of P4-P6 variant A225U in 2.0 M RbCl. The intensities of the donor dye (green) and the acceptor dye (red) are shown. The black line denotes the probability of the high FRET state determined by a hidden Markov model fit to the data. ${ }^{6}$ To scale the length of the randomly selected FRET traces and ease their comparison, traces were truncated at the length of the shortest selected trace. 
A

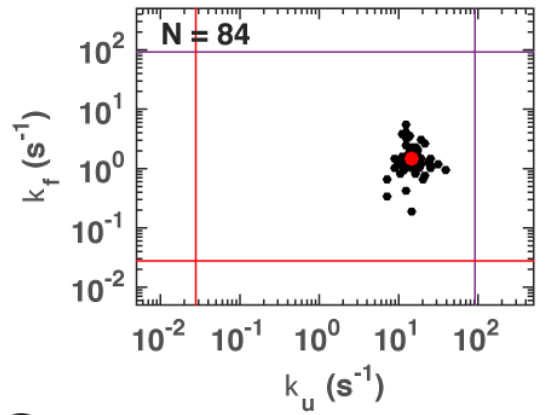

C

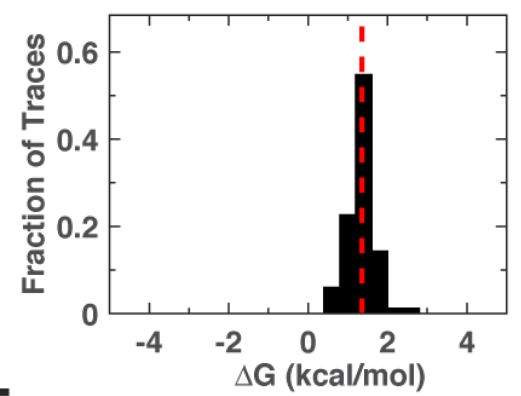

$\mathbf{E}$

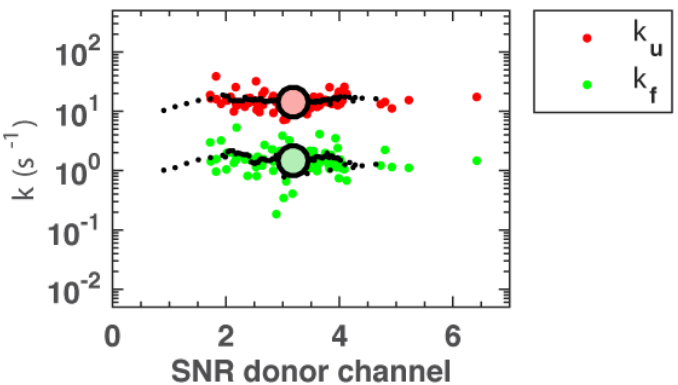

B

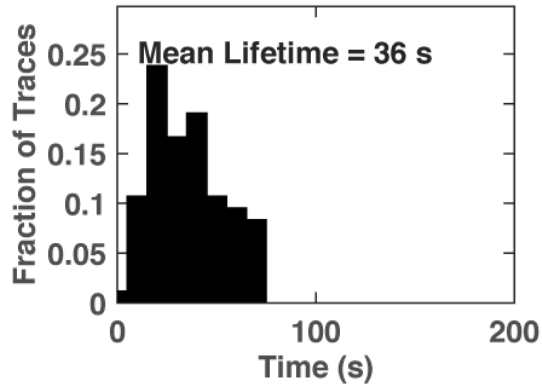

D
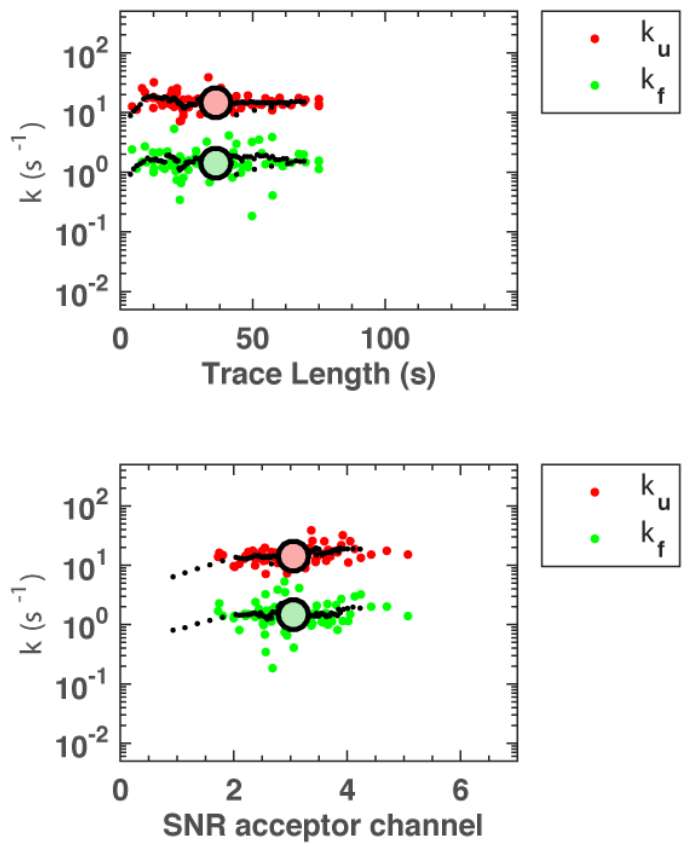

Figure S11-1. smFRET data for P4-P6 A225U in 2.5 M RbCl. A) Scatter plot of fitted values of $k_{\mathrm{f}}$ versus $k_{\mathrm{u}}$ for each molecule. ${ }^{6}$ Purple lines indicate the camera frame rate and red lines indicate the average lifetime of the molecules. The red dot indicates mean folding and unfolding rate constants. TIRF measurements were taken at 91 frames per second (see Experimental Methods in main text). $\mathrm{N}=$ number of molecules. B) Histogram of trace lengths for individual molecules. C) Histogram of $\Delta \mathrm{G}$ values for individual molecules (determined from equilibrium $K_{e q}=k_{f} / k_{u}$ ). The mean value is denoted by the dashed red line. D) Scatter plot of rate constants versus trace length; $k_{\mathrm{f}}$ (green) and $k_{\mathrm{u}}$ (red). The mean value for each rate constant is presented by larger light green $\left(k_{\mathrm{f}}\right)$ and red $\left(k_{\mathrm{u}}\right)$ circles. The black dots are the average of ten values and are shown to provide an empirical guide. E) and F) Rate constants as a function of signal-to-noise ratio (SNR) in the donor channel and acceptor channels respectively. Colors as in (D). 
A

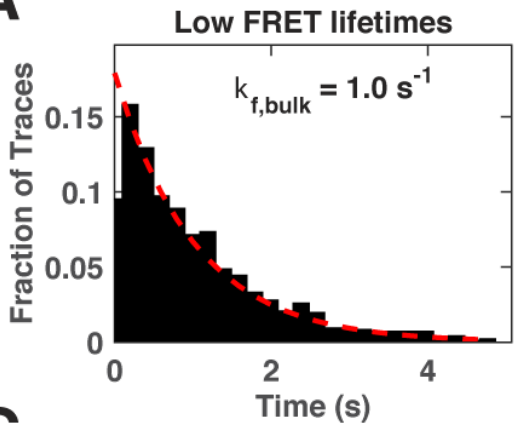

Bulk FRET Distribution

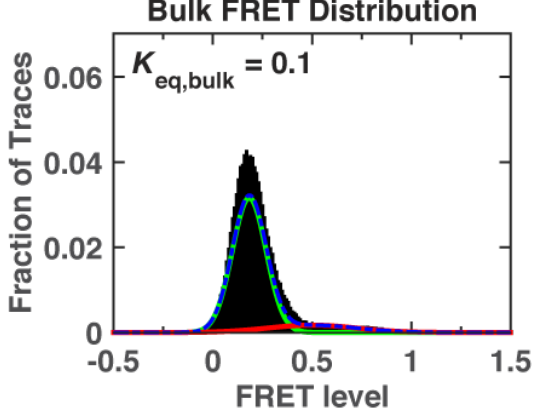

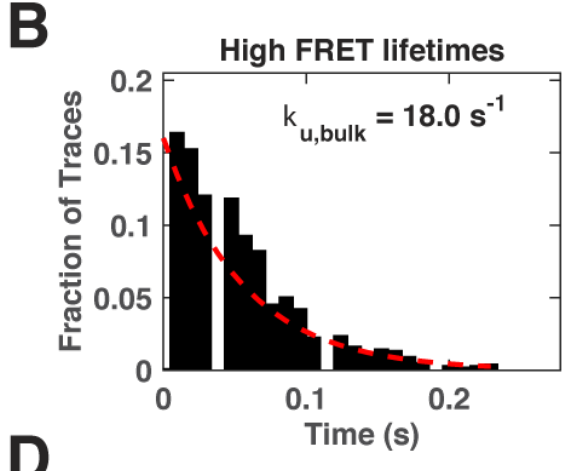

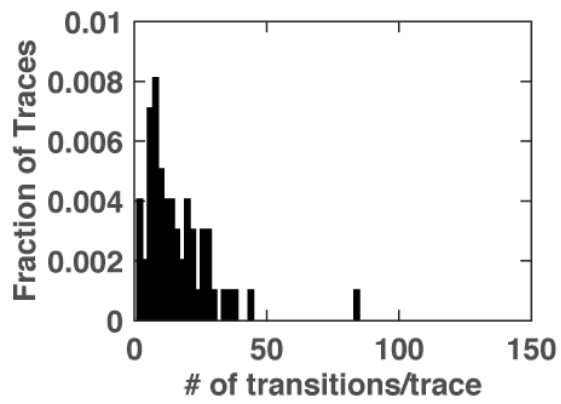

Figure S11-2. smFRET data of P4-P6 variant A225U in 2.5 M RbCl. A) and B) Distribution of the $k_{\mathrm{f}}$ (folding) and $k_{\mathrm{u}}$ (unfolding) rate constants as a function of time. An exponential fit (dashed red lines) to the distribution of lifetimes in the unfolded (A) or folded (B) states of all molecules was used to determine $k_{\mathrm{f}}(\mathrm{A})$ and $k_{\mathrm{u}}(\mathrm{B})$ rate constants (see Experimental Methods in main text). C) Histogram of FRET distribution for all molecules. Distribution is fit to a two-Gaussian model (blue). The equilibrium constant $\left(K_{\text {eq,bulk }}\right)$ was determined from the ratio of the fraction of molecules that are in the high FRET peak (red) versus low FRET peak (green). D) Distribution of the number of transitions per trace. 

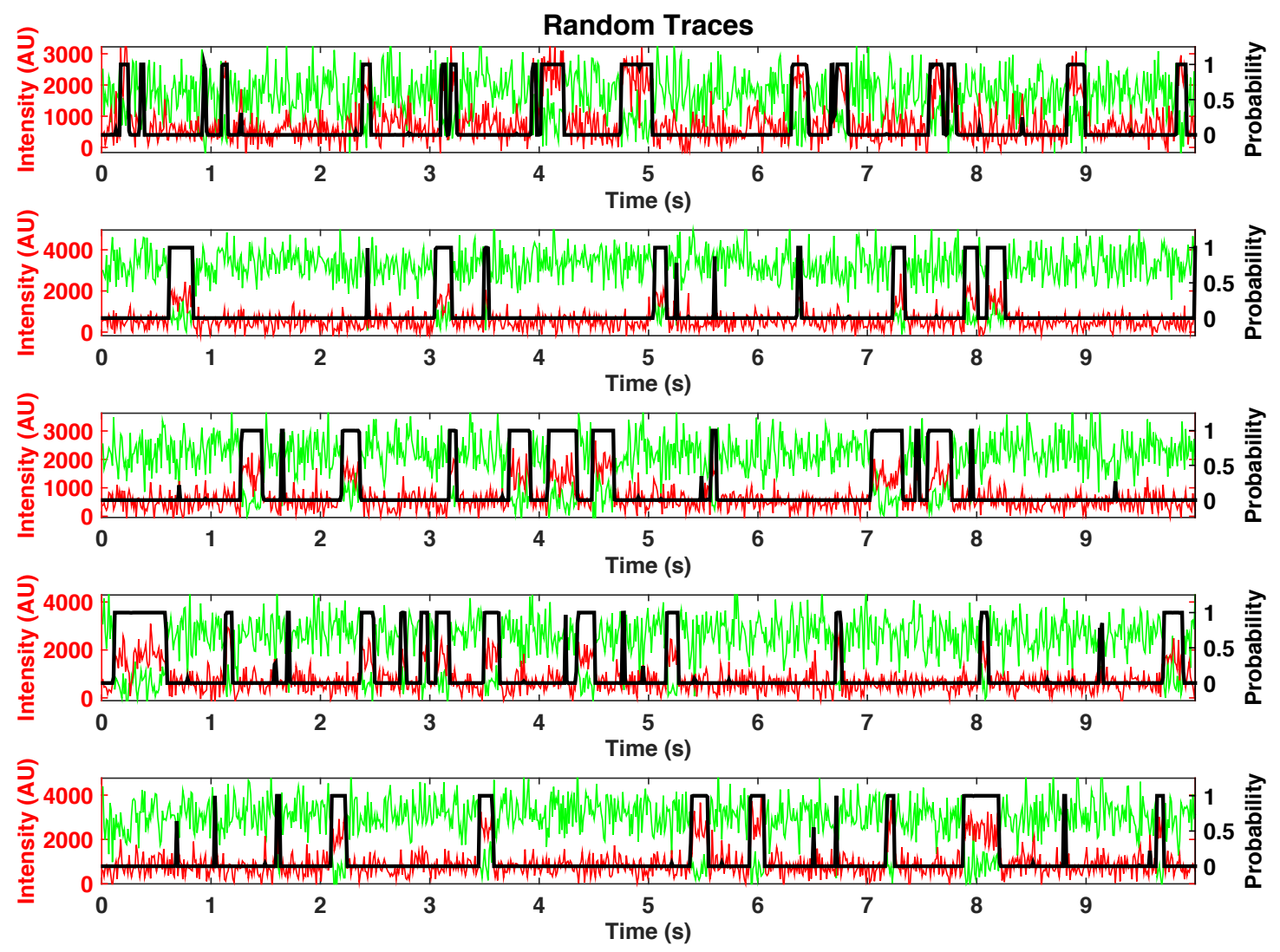

Figure S11-3. Randomly selected FRET traces of P4-P6 variant A225U in $2.5 \mathbf{~ M ~ R b C l}$. The intensities of the donor dye (green) and the acceptor dye (red) are shown. The black line denotes the probability of the high FRET state determined by a hidden Markov model fit to the data. ${ }^{6}$ To scale the length of the randomly selected FRET traces and ease their comparison, traces were truncated at the length of the shortest selected trace. 
A

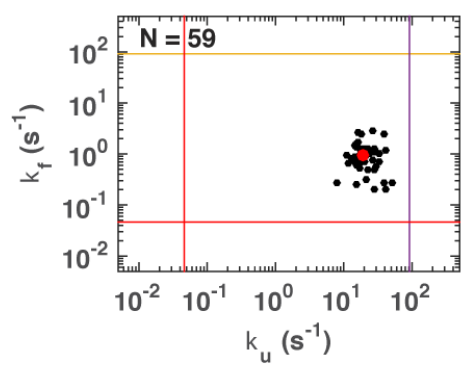

C

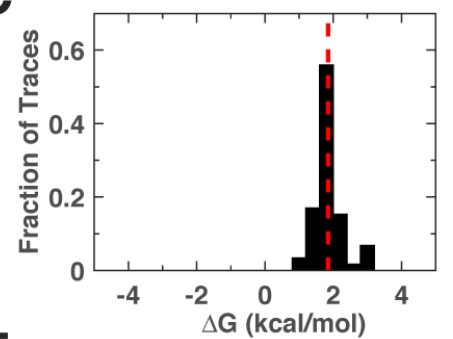

E

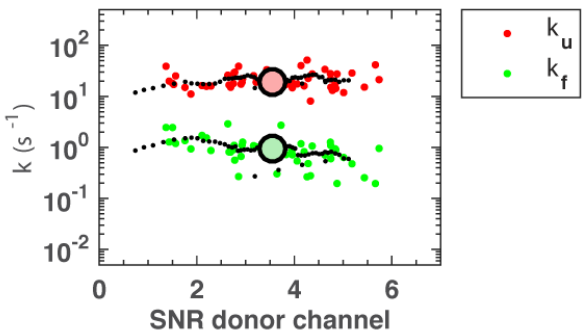

B

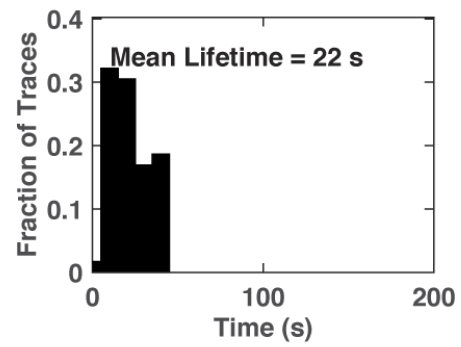

D

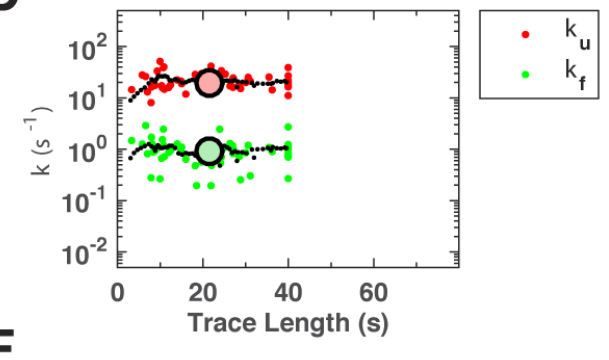

$\mathbf{F}$

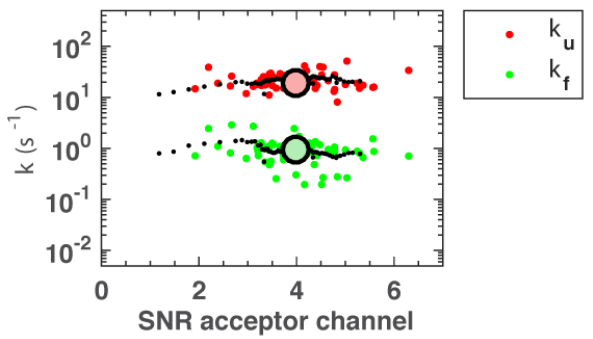

Figure S12-1. smFRET data for P4-P6 A225U in 2.8 M RbCl. A) Scatter plot of fitted values of $k_{\mathrm{f}}$ versus $k_{\mathrm{u}}$ for each molecule. ${ }^{6}$ Purple lines indicate the camera frame rate and red lines indicate the average lifetime of the molecules. The red dot indicates mean folding and unfolding rate constants. TIRF measurements were taken at 91 frames per second (see Experimental Methods in main text). $\mathrm{N}=$ number of molecules. B) Histogram of trace lengths for individual molecules. C) Histogram of $\Delta \mathrm{G}$ values for individual molecules (determined from equilibrium $K_{e q}=k_{f} / k_{u}$ ). The mean value is denoted by the dashed red line. D) Scatter plot of rate constants versus trace length; $k_{\mathrm{f}}$ (green) and $k_{\mathrm{u}}$ (red). The mean value for each rate constant is presented by larger light green $\left(k_{\mathrm{f}}\right)$ and red $\left(k_{\mathrm{u}}\right)$ circles. The black dots are the average of ten values and are shown to provide an empirical guide. E) and F) Rate constants as a function of signal-to-noise ratio (SNR) in the donor channel and acceptor channels respectively. Colors as in (D). 
A

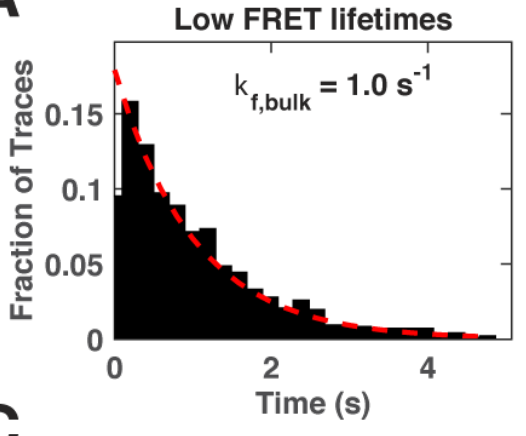

Bulk FRET Distribution

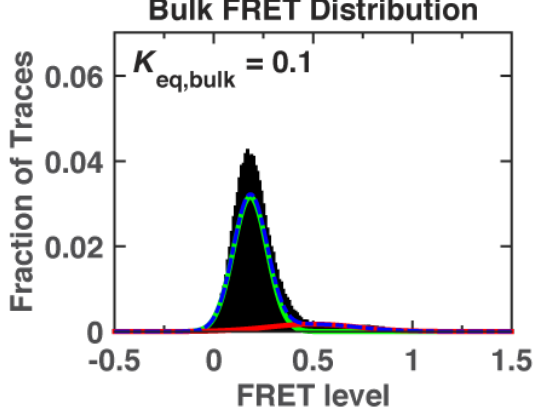

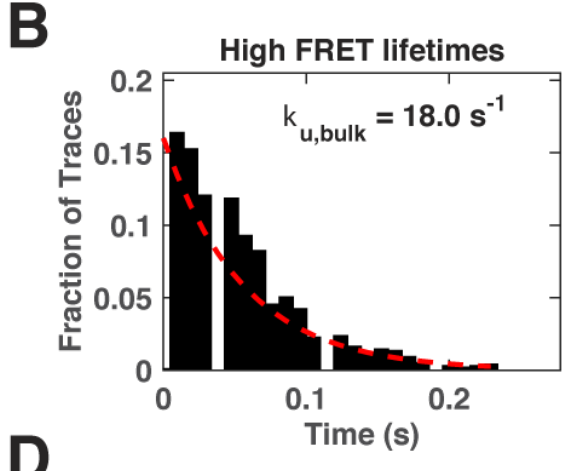

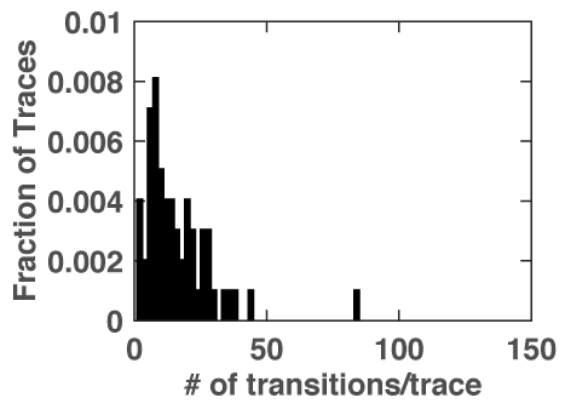

Figure S12-2. smFRET data of P4-P6 variant A225U in $2.8 \mathbf{~ M ~ R b C l}$. A) and B) Distribution of the $k_{\mathrm{f}}$ (folding) and $k_{\mathrm{u}}$ (unfolding) rate constants as a function of time. An exponential fit (dashed red lines) to the distribution of lifetimes in the unfolded (A) or folded (B) states of all molecules was used to determine $k_{\mathrm{f}}(\mathrm{A})$ and $k_{\mathrm{u}}(\mathrm{B})$ rate constants (see Experimental Methods in main text). C) Histogram of FRET distribution for all molecules. Distribution is fit to a two-Gaussian model (blue). The equilibrium constant $\left(K_{\text {eq,bulk }}\right)$ was determined from the ratio of the fraction of molecules that are in the high FRET peak (red) versus low FRET peak (green). D) Distribution of the number of transitions per trace. 

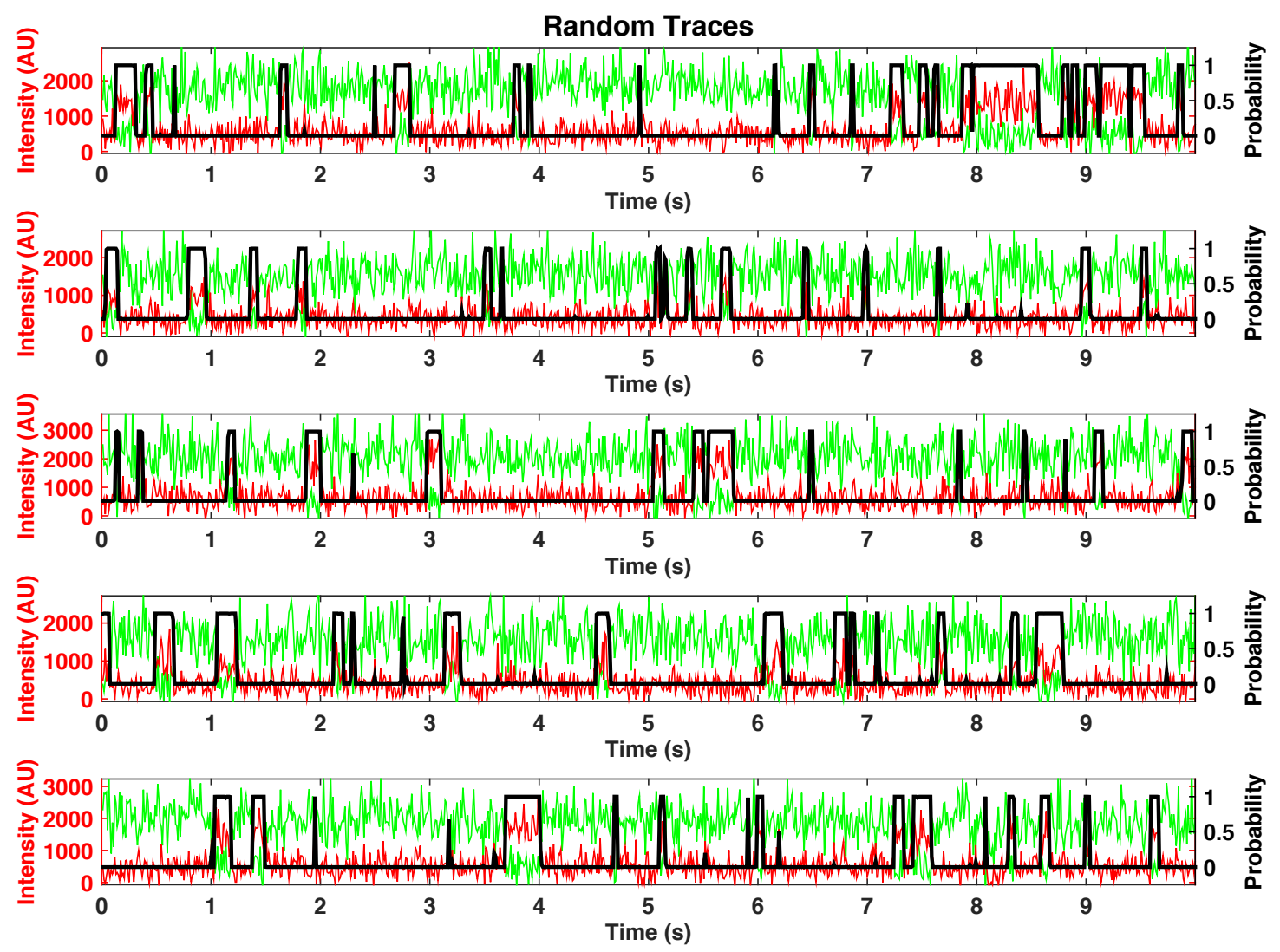

Figure S12-3. Randomly selected FRET traces of P4-P6 variant A225U in $2.8 \mathbf{~ M ~ R b C l}$. The intensities of the donor dye (green) and the acceptor dye (red) are shown. The black line denotes the probability of the high FRET state determined by a hidden Markov model fit to the data. ${ }^{6}$ To scale the length of the randomly selected FRET traces and ease their comparison, traces were truncated at the length of the shortest selected trace. 
A

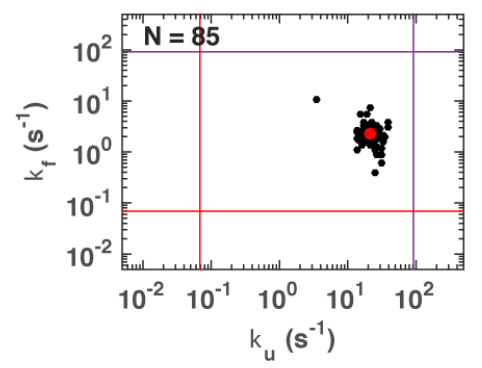

C

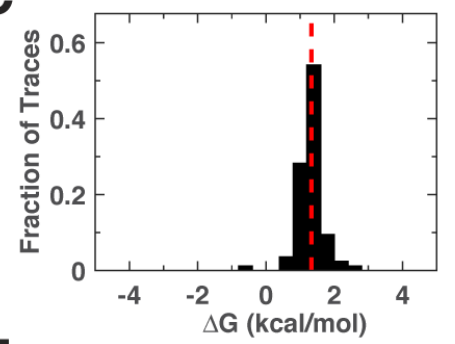

$\mathbf{E}$

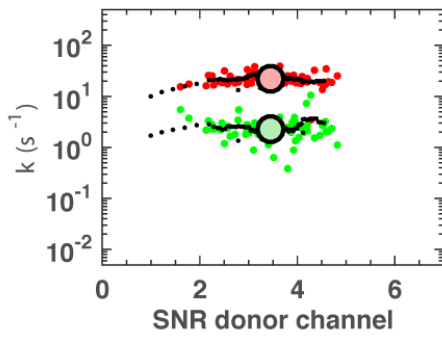

B

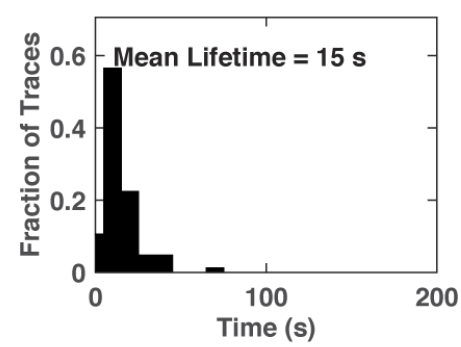

D

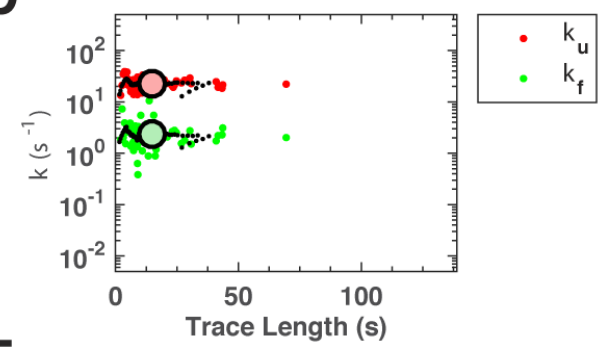

$\mathbf{F}$

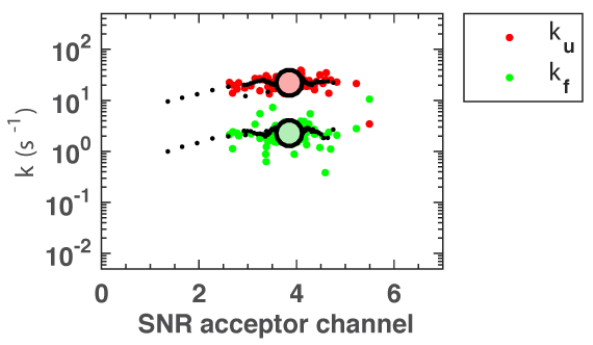

Figure S13-1. smFRET data for P4-P6 A225U in 1.8 M RbF. A) Scatter plot of fitted values of $k_{\mathrm{f}}$ versus $k_{\mathrm{u}}$ for each molecule. ${ }^{6}$ Purple lines indicate the camera frame rate and red lines indicate the average lifetime of the molecules. The red dot indicates mean folding and unfolding rate constants. TIRF measurements were taken at 91 frames per second (see Experimental Methods in main text). $\mathrm{N}=$ number of molecules. B) Histogram of trace lengths for individual molecules. C) Histogram of $\Delta \mathrm{G}$ values for individual molecules (determined from equilibrium $K_{e q}=k_{f} / k_{u}$ ). The mean value is denoted by the dashed red line. D) Scatter plot of rate constants versus trace length; $k_{\mathrm{f}}$ (green) and $k_{\mathrm{u}}$ (red). The mean value for each rate constant is presented by larger light green $\left(k_{\mathrm{f}}\right)$ and red $\left(k_{\mathrm{u}}\right)$ circles. The black dots are the average of ten values and are shown to provide an empirical guide. E) and F) Rate constants as a function of signal-to-noise ratio (SNR) in the donor channel and acceptor channels respectively. Colors as in (D). 
A
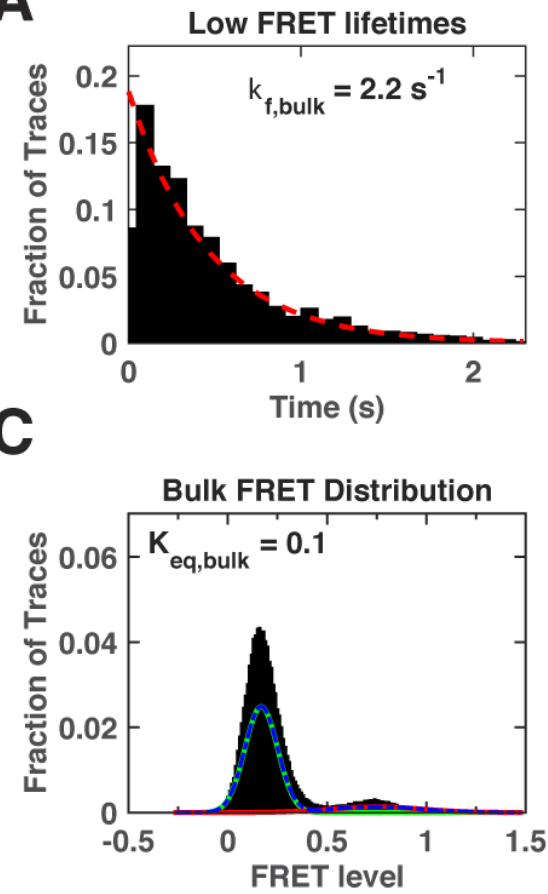

B
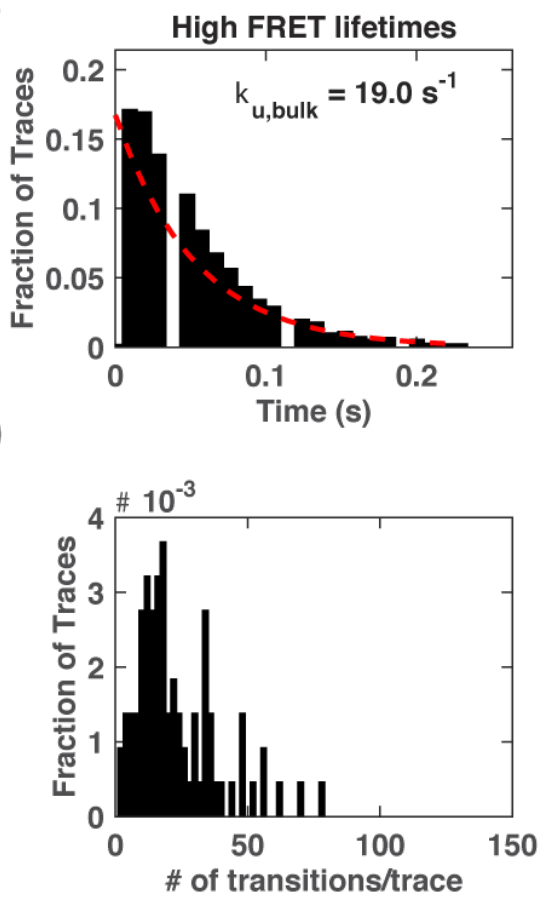

Figure S13-2. smFRET data of P4-P6 variant A225U in 1.8 M RbF. A) and B) Distribution of the $k_{\mathrm{f}}$ (folding) and $k_{\mathrm{u}}$ (unfolding) rate constants as a function of time. An exponential fit (dashed red lines) to the distribution of lifetimes in the unfolded (A) or folded (B) states of all molecules was used to determine $k_{\mathrm{f}}(\mathrm{A})$ and $k_{\mathrm{u}}(\mathrm{B})$ rate constants (see Experimental Methods in main text). C) Histogram of FRET distribution for all molecules. Distribution is fit to a two-Gaussian model (blue). The equilibrium constant $\left(K_{\text {eq,bulk }}\right)$ was determined from the ratio of the fraction of molecules that are in the high FRET peak (red) versus low FRET peak (green). D) Distribution of the number of transitions per trace. 

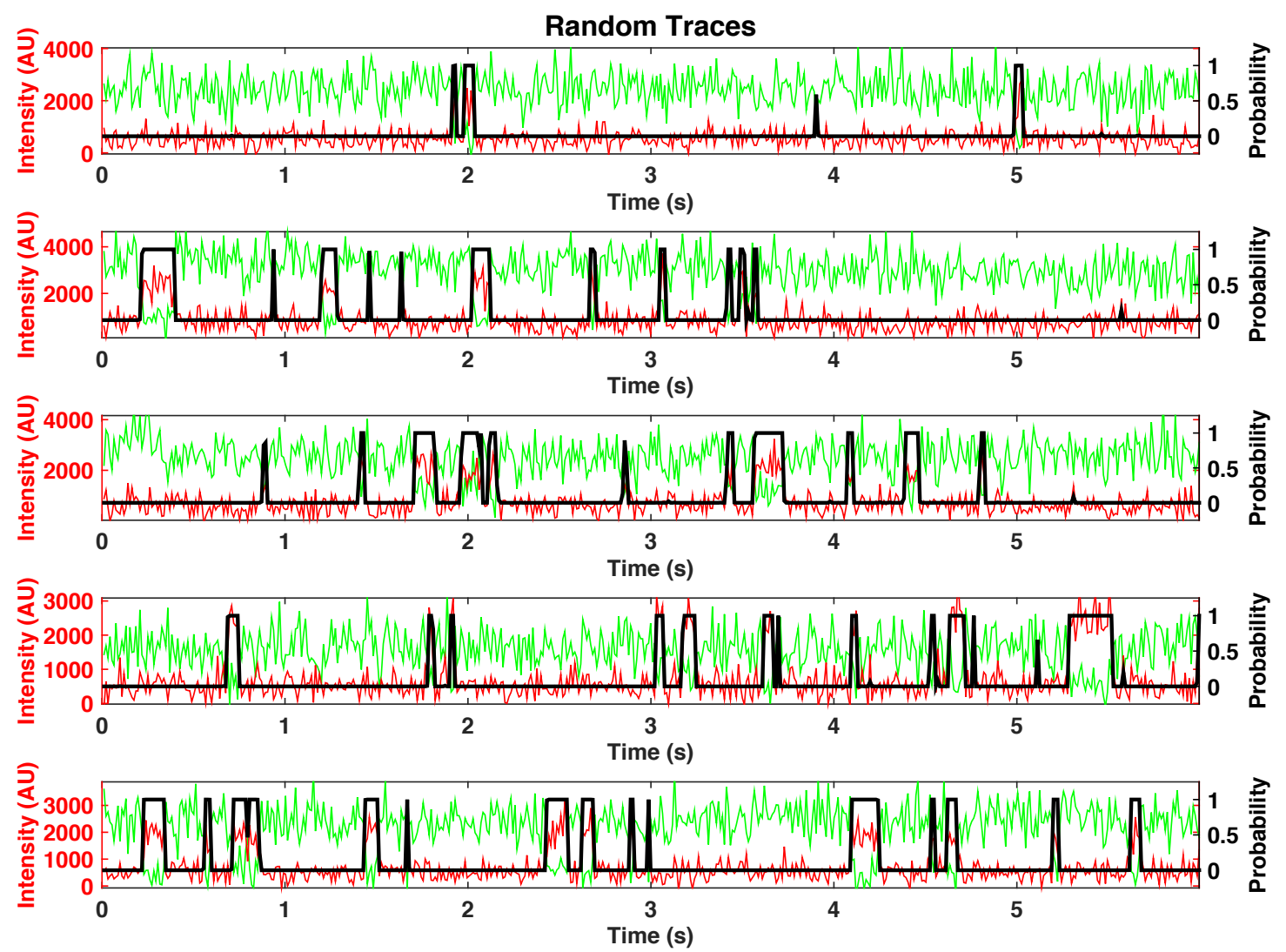

Figure S13-3. Randomly selected FRET traces of P4-P6 variant A225U in 1.8 M RbF. The intensities of the donor dye (green) and the acceptor dye (red) are shown. The black line denotes the probability of the high FRET state determined by a hidden Markov model fit to the data. ${ }^{6}$ To scale the length of the randomly selected FRET traces and ease their comparison, traces were truncated at the length of the shortest selected trace. 
A

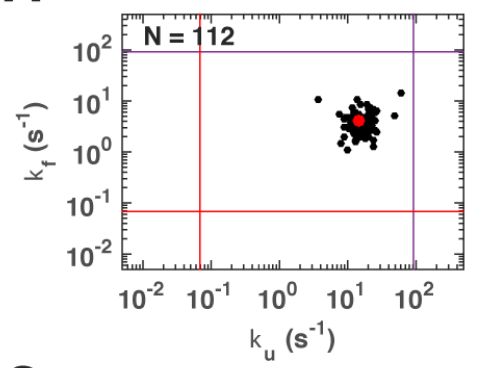

C

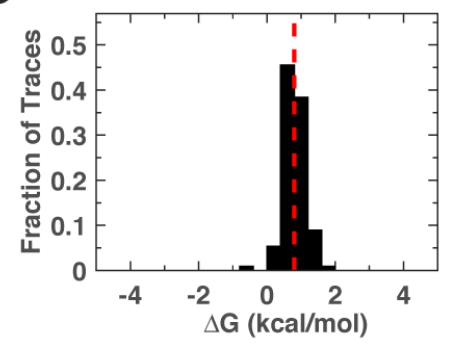

$\mathbf{E}$

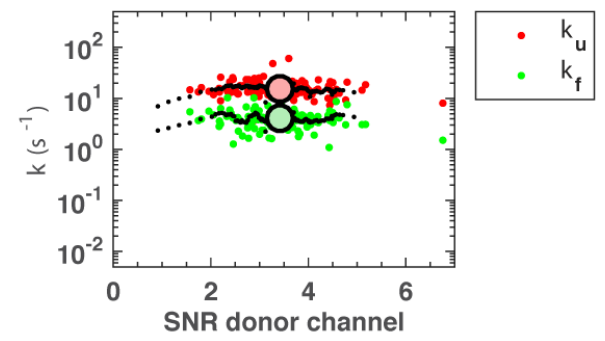

B

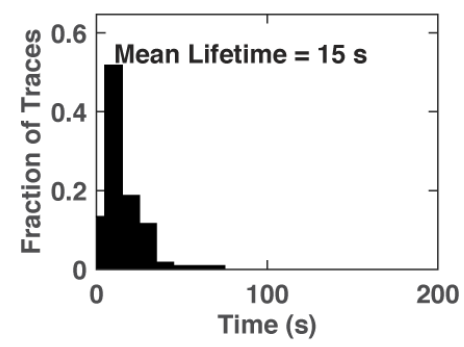

D

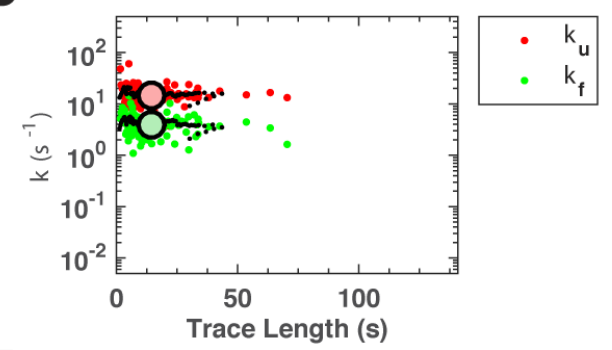

$\mathbf{F}$

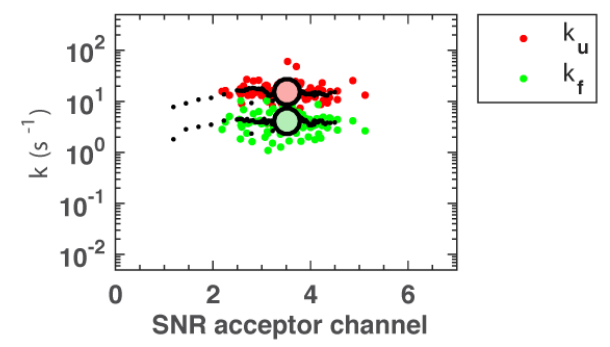

Figure S14-1. smFRET data for P4-P6 A225U in 2.3 M RbF. A) Scatter plot of fitted values of $k_{\mathrm{f}}$ versus $k_{\mathrm{u}}$ for each molecule. ${ }^{6}$ Purple lines indicate the camera frame rate and red lines indicate the average lifetime of the molecules. The red dot indicates mean folding and unfolding rate constants. TIRF measurements were taken at 91 frames per second (see Experimental Methods in main text). $\mathrm{N}=$ number of molecules. B) Histogram of trace lengths for individual molecules. C) Histogram of $\Delta \mathrm{G}$ values for individual molecules (determined from equilibrium $K_{e q}=k_{f} / k_{u}$ ). The mean value is denoted by the dashed red line. D) Scatter plot of rate constants versus trace length; $k_{\mathrm{f}}$ (green) and $k_{\mathrm{u}}$ (red). The mean value for each rate constant is presented by larger light green $\left(k_{\mathrm{f}}\right)$ and red $\left(k_{\mathrm{u}}\right)$ circles. The black dots are the average of ten values and are shown to provide an empirical guide. E) and F) Rate constants as a function of signal-to-noise ratio (SNR) in the donor channel and acceptor channels respectively. Colors as in (D). 
A
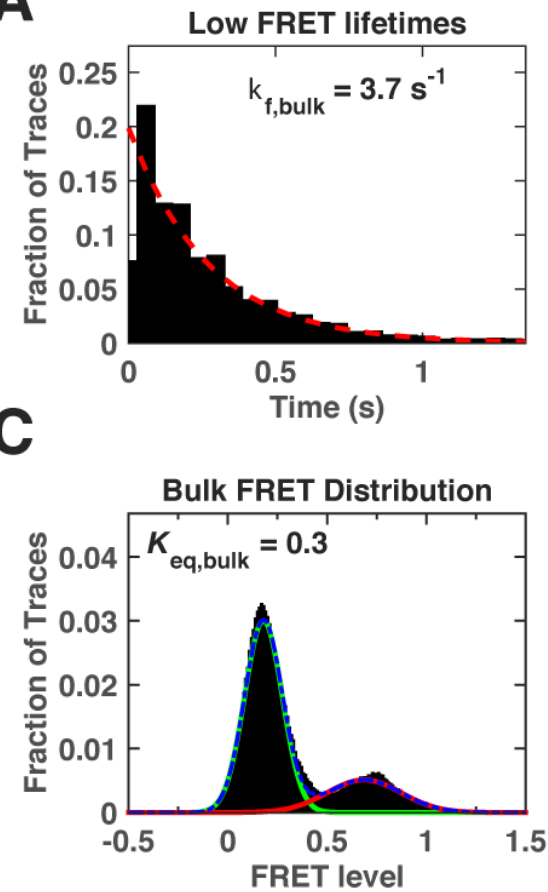

B

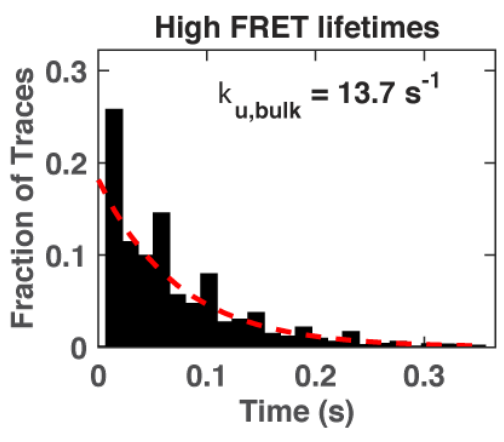

D

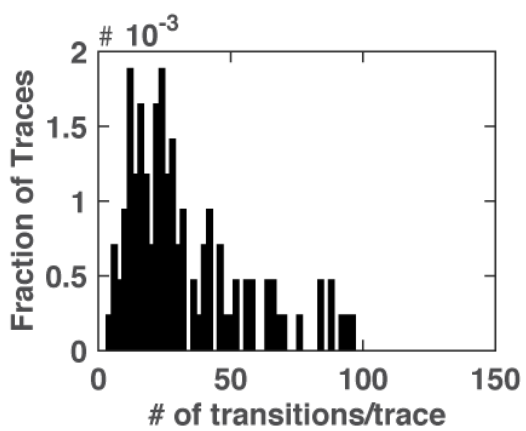

Figure S14-2. smFRET data of P4-P6 variant A225U in 2.3 M RbF. A) and B) Distribution of the $k_{\mathrm{f}}$ (folding) and $k_{\mathrm{u}}$ (unfolding) rate constants as a function of time. An exponential fit (dashed red lines) to the distribution of lifetimes in the unfolded (A) or folded (B) states of all molecules was used to determine $k_{\mathrm{f}}(\mathrm{A})$ and $k_{\mathrm{u}}(\mathrm{B})$ rate constants (see Experimental Methods in main text). C) Histogram of FRET distribution for all molecules. Distribution is fit to a two-Gaussian model (blue). The equilibrium constant $\left(K_{\text {eq,bulk }}\right)$ was determined from the ratio of the fraction of molecules that are in the high FRET peak (red) versus low FRET peak (green). D) Distribution of the number of transitions per trace. 

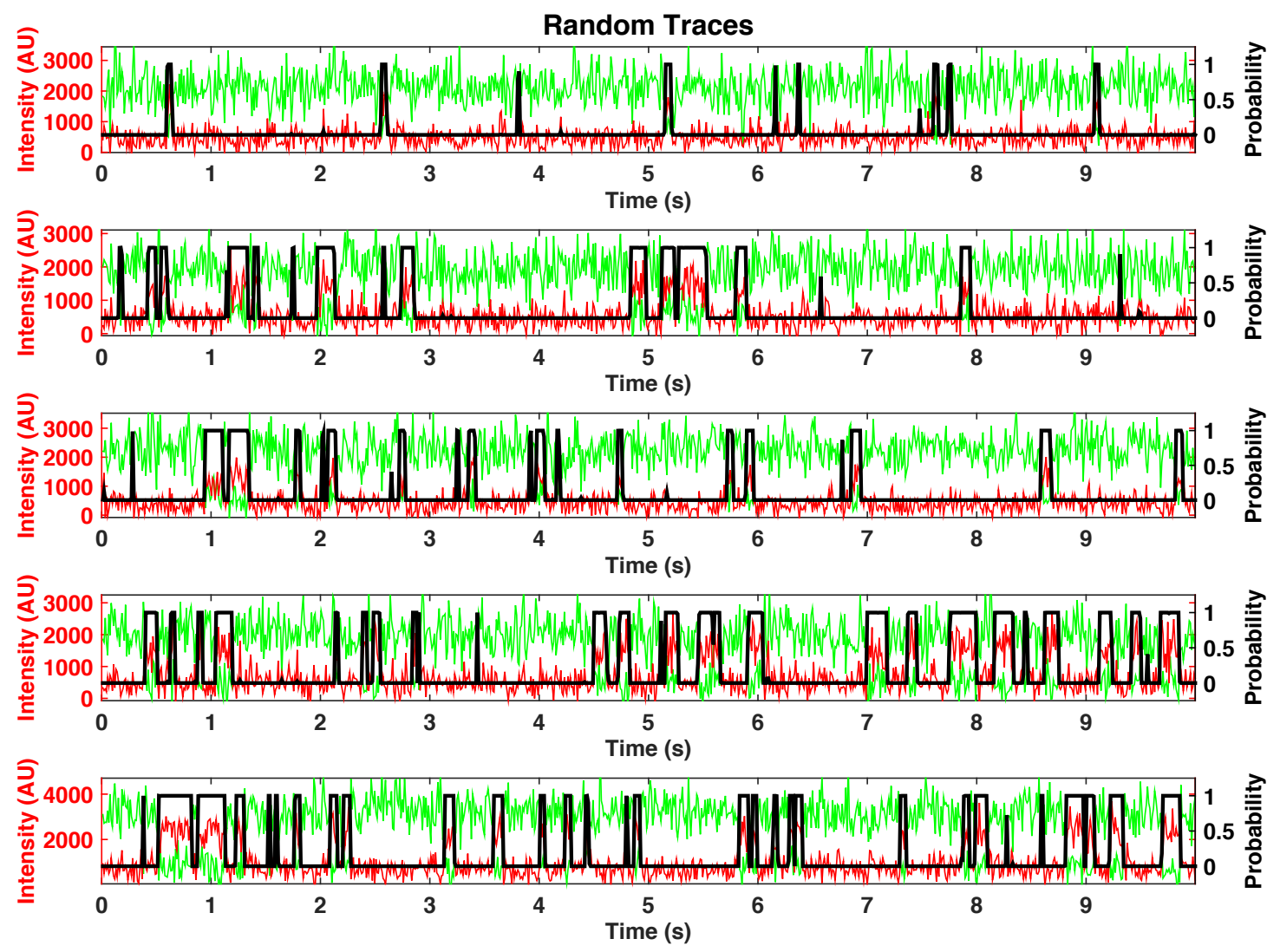

Figure S14-3. Randomly selected FRET traces of P4-P6 variant A225U in 2.3 M RbF. The intensities of the donor dye (green) and the acceptor dye (red) are shown. The black line denotes the probability of the high FRET state determined by a hidden Markov model fit to the data. ${ }^{6}$ To scale the length of the randomly selected FRET traces and ease their comparison, traces were truncated at the length of the shortest selected trace. 
A

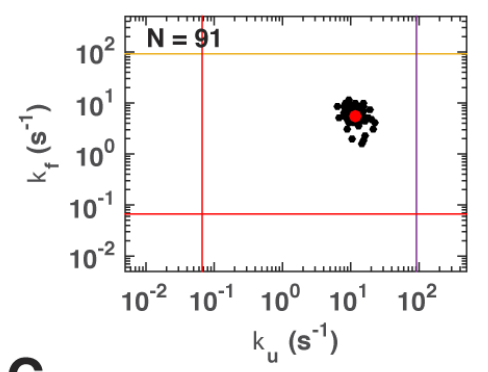

C

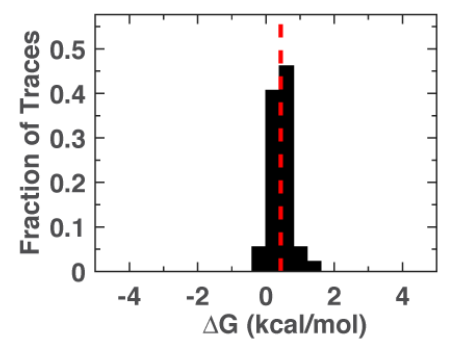

E

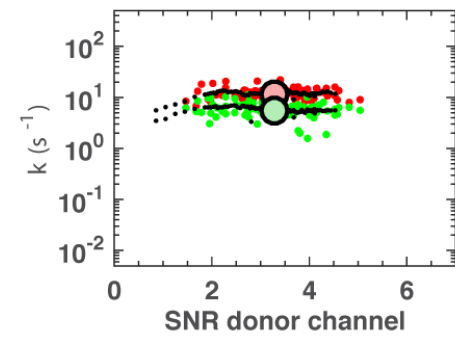

B

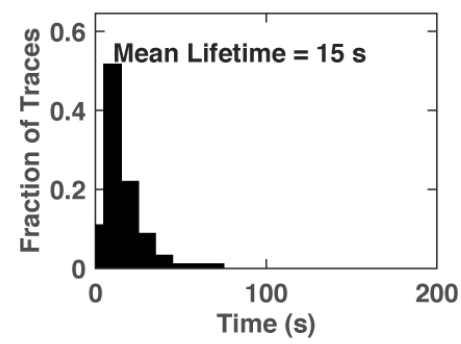

D

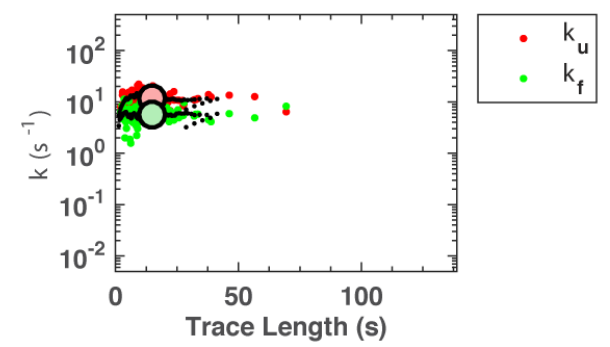

$\mathbf{F}$

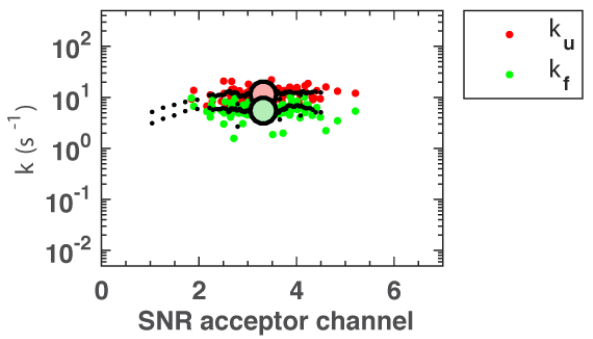

Figure S15-1. smFRET data for P4-P6 A225U in 2.5 M RbF. A) Scatter plot of fitted values of $k_{\mathrm{f}}$ versus $k_{\mathrm{u}}$ for each molecule. ${ }^{6}$ Purple lines indicate the camera frame rate and red lines indicate the average lifetime of the molecules. The red dot indicates mean folding and unfolding rate constants. TIRF measurements were taken at 91 frames per second (see Experimental Methods in main text). $\mathrm{N}=$ number of molecules. B) Histogram of trace lengths for individual molecules. C) Histogram of $\Delta \mathrm{G}$ values for individual molecules (determined from equilibrium $K_{e q}=k_{f} / k_{u}$ ). The mean value is denoted by the dashed red line. D) Scatter plot of rate constants versus trace length; $k_{\mathrm{f}}$ (green) and $k_{\mathrm{u}}$ (red). The mean value for each rate constant is presented by larger light green $\left(k_{\mathrm{f}}\right)$ and red $\left(k_{\mathrm{u}}\right)$ circles. The black dots are the average of ten values and are shown to provide an empirical guide. E) and F) Rate constants as a function of signal-to-noise ratio (SNR) in the donor channel and acceptor channels respectively. Colors as in (D). 
A
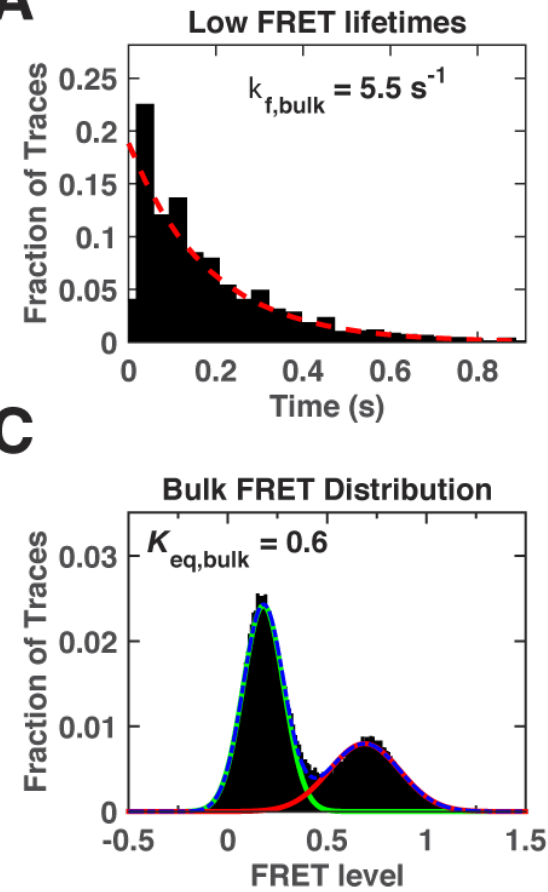
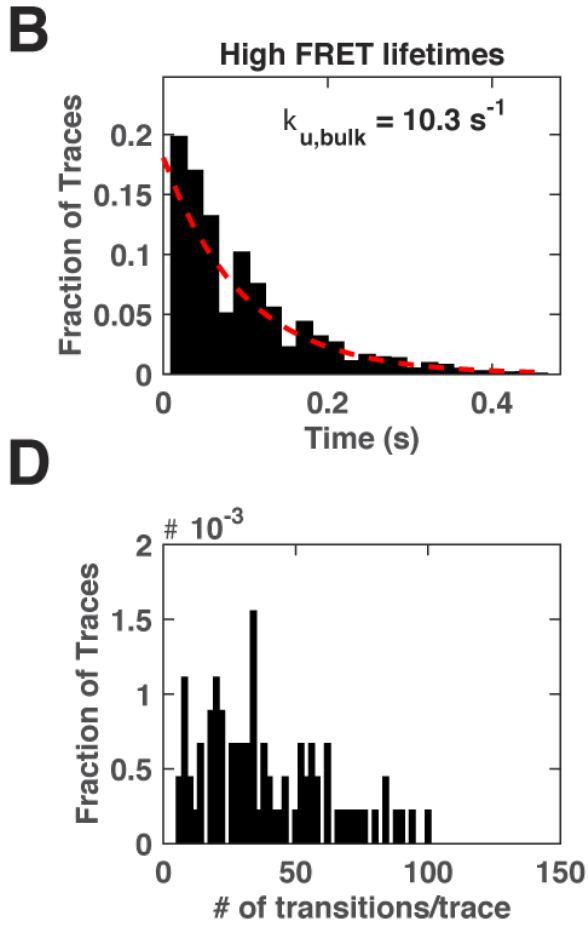

Figure S15-2. smFRET data of P4-P6 variant A225U in 2.5 M RbF. A) and B) Distribution of the $k_{\mathrm{f}}$ (folding) and $k_{\mathrm{u}}$ (unfolding) rate constants as a function of time. An exponential fit (dashed red lines) to the distribution of lifetimes in the unfolded (A) or folded (B) states of all molecules was used to determine $k_{\mathrm{f}}(\mathrm{A})$ and $k_{\mathrm{u}}(\mathrm{B})$ rate constants (see Experimental Methods in main text). C) Histogram of FRET distribution for all molecules. Distribution is fit to a two-Gaussian model (blue). The equilibrium constant $\left(K_{\text {eq,bulk }}\right)$ was determined from the ratio of the fraction of molecules that are in the high FRET peak (red) versus low FRET peak (green). D) Distribution of the number of transitions per trace. 

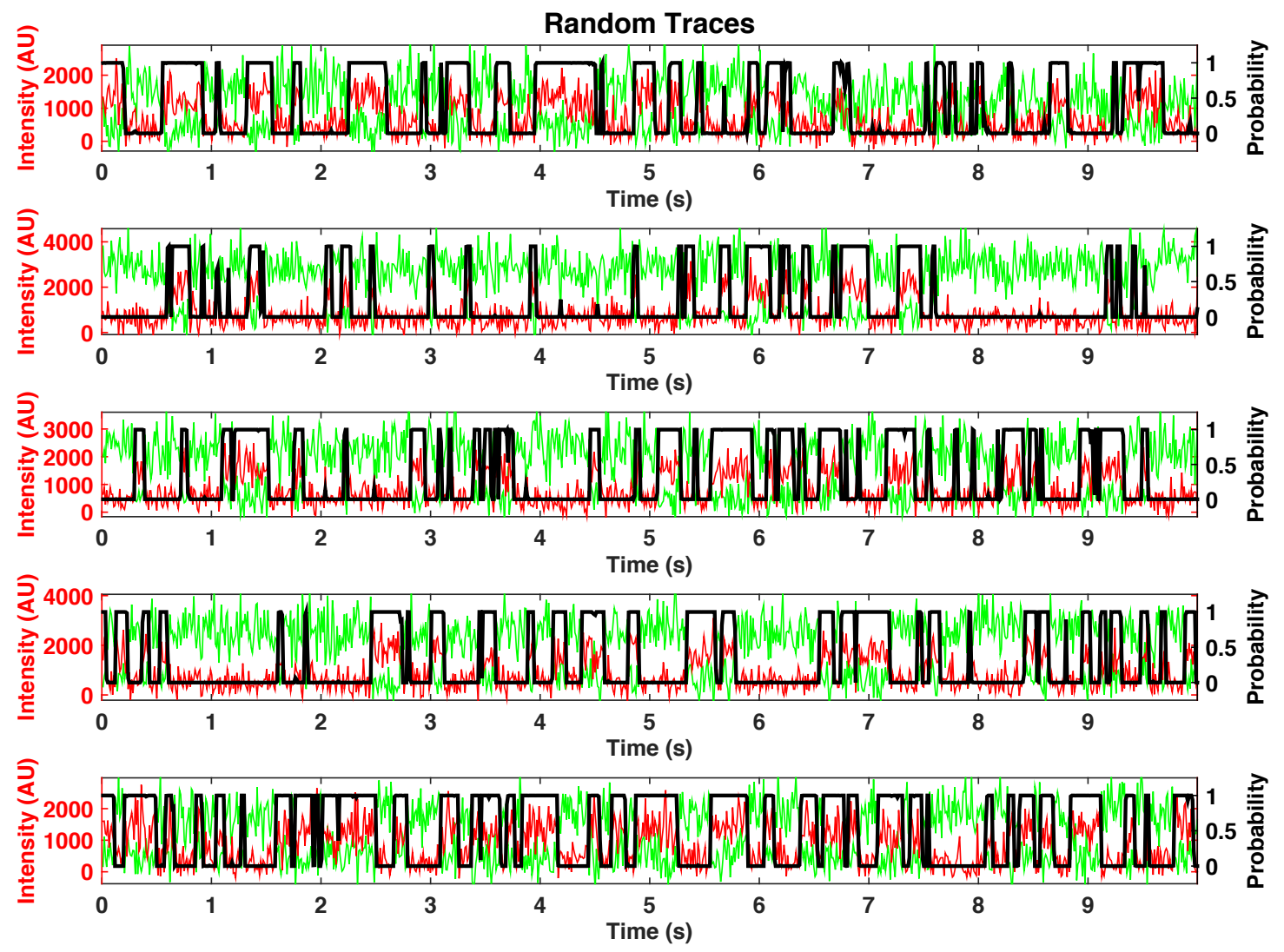

Figure S15-3. Randomly selected FRET traces of P4-P6 variant A225U in 2.5 M RbF. The intensities of the donor dye (green) and the acceptor dye (red) are shown. The black line denotes the probability of the high FRET state determined by a hidden Markov model fit to the data. ${ }^{6}$ To scale the length of the randomly selected FRET traces and ease their comparison, traces were truncated at the length of the shortest selected trace. 

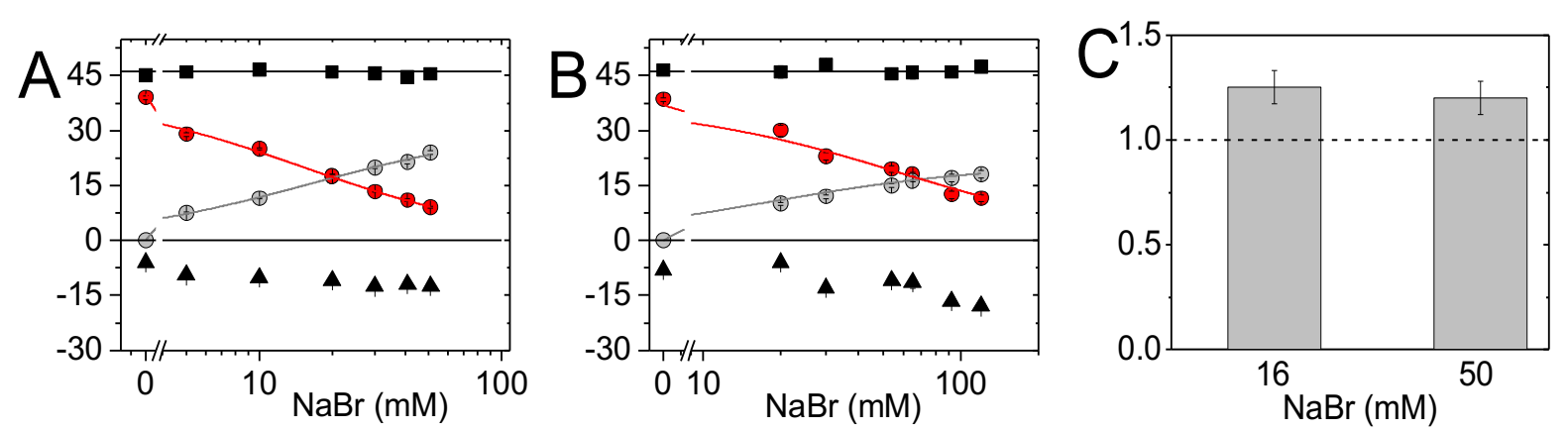

Figure S16. Competitive association of monovalent cations with a 24-bp DNA duplex. A and B) The number of ions in the ion atmosphere for $\mathrm{Na}^{+}$(gray circles) versus $\mathrm{Li}^{+}$(red circles; A) $\left[\mathrm{Li}^{+}\right]=16 \mathrm{mM}$, B) $\left[\mathrm{Li}^{+}\right]=50 \mathrm{mM}$ ); excluded $\mathrm{Br}^{-}$anion is represented by the black triangles. The total charge of the ion atmosphere summed from the individual ion measurements is shown as the black squares, and the lines at $\Gamma=+46$ represent the charge needed to neutralize the total DNA charge of -46 . Solid lines (red and gray) are fits with the Hill equation and provide an empirical guide. Each data point is the average of 3-5 independent measurements. Errors are the standard deviation of all measurements and shown when not smaller than the symbols. C) Cation competition constants for $\mathrm{Na}^{+}$against $\mathrm{Li}^{+}$. See Tables $\mathrm{S} 9$ and S10 for data. 

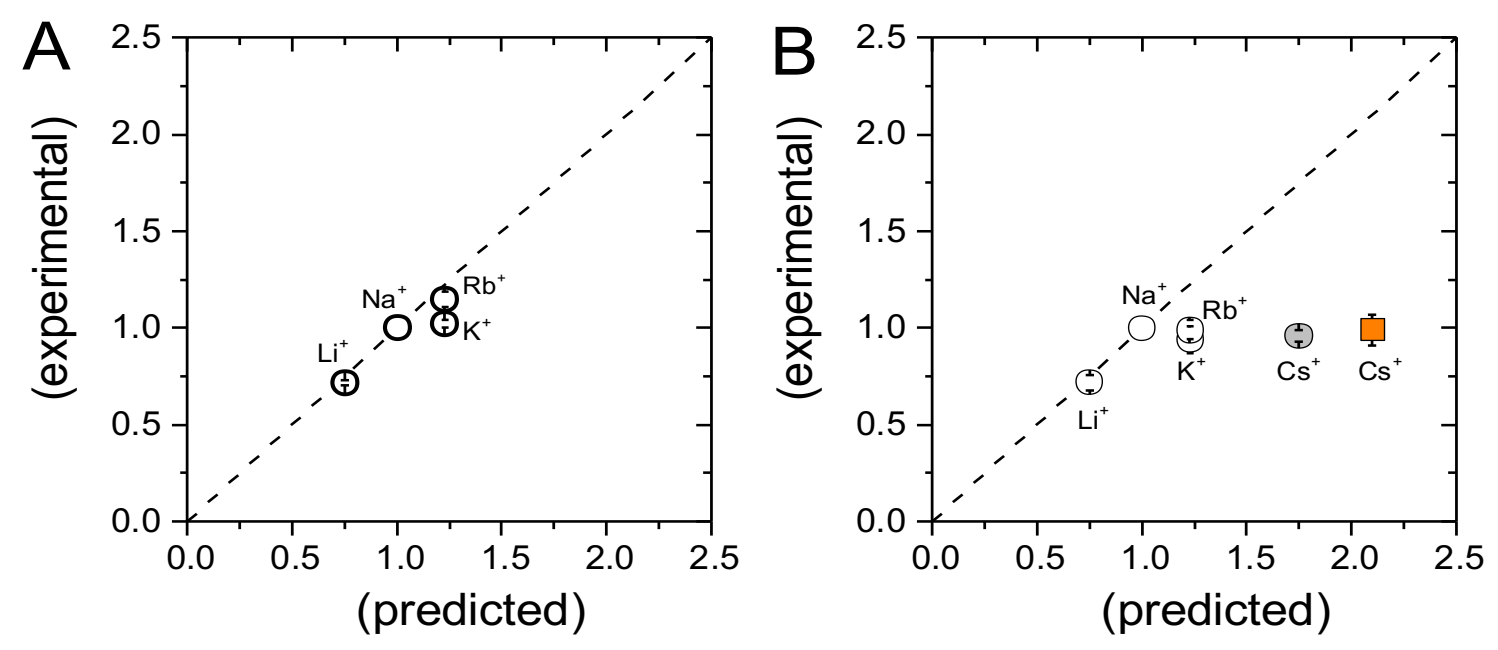

Figure S17. Comparison of experimentally determined versus 3D-RISM computationally determined ion atmosphere cation competition ( $\alpha$ values; eq 5 in main text). A) Comparison between experimental $\alpha$ estimated from results in ref 1 and computational $\alpha$ values from ref 7. B) Comparison between experimental $\alpha$ obtained herein and computational $\alpha$ values from ref 7. (Reproduced from Figure 6 from main text for comparison.) Cations that were computationally estimated, subsequent to prior experimental measurements, ${ }^{1}$ are shown with open symbols, and those with blind computational predictions ${ }^{7}$ by closed symbols. The open and gray symbols are for a 24-bp DNA, and the orange symbol for a 24-bp RNA. Each cation (identified in figures) was competed versus $\mathrm{Na}^{+}$, so that $\alpha$ is 1 for $\mathrm{Na}^{+}$and there is no error. 

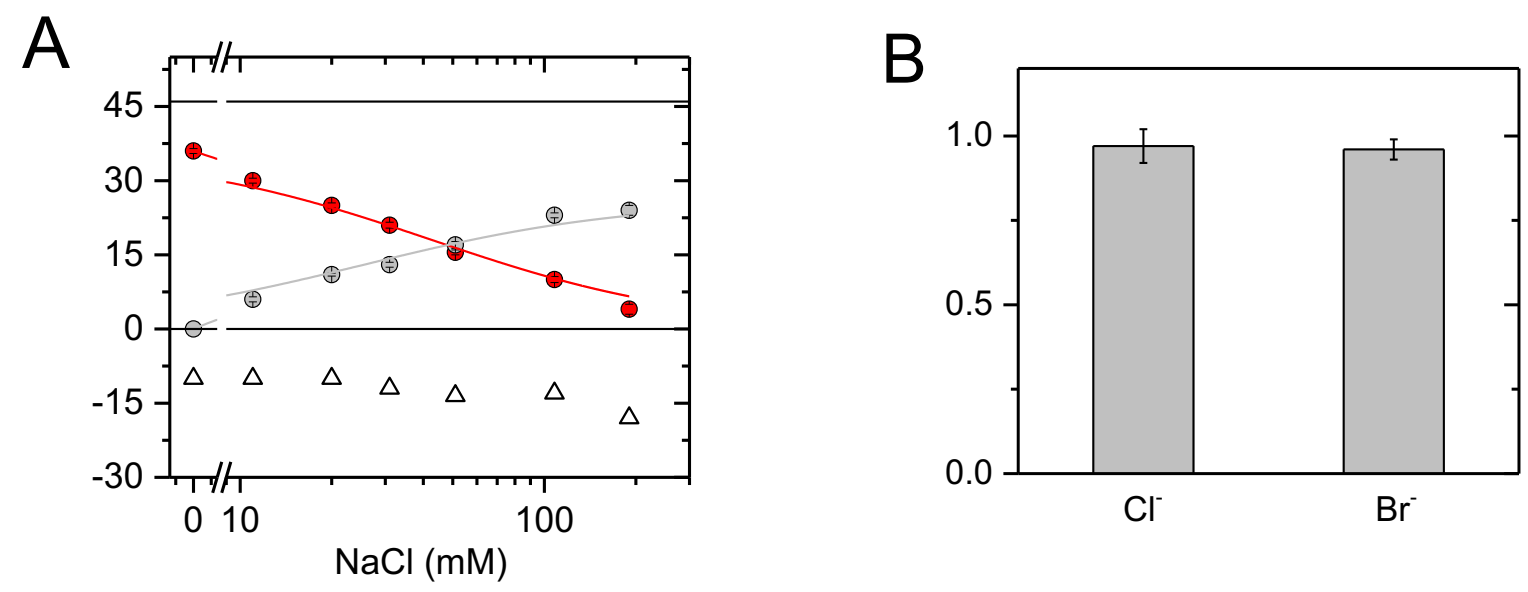

Figure S18. Competitive association of monovalent cations with a 24-bp DNA duplex with $\mathrm{Cl}^{-}$as the coion. A) The number of ions in the ion atmosphere for $\mathrm{Cs}^{+}$(gray circles) versus $\mathrm{Na}^{+}$(red circles; $\left[\mathrm{Na}^{+}\right]=$ $46 \mathrm{mM}$ ); excluded $\mathrm{Cl}^{-}$anions are represented by the open triangles and were estimated based on eq 1 (in main text). The black solid lines at $\Gamma=+46$ represent the charge needed to neutralize the total DNA charge of -46 . Solid lines (red and gray) are fits with the Hill equation and provide an empirical guide. Each data point is the average of two independent measurements. Errors are the standard deviation of all measurements. B) Comparison of cation competition constants ( $\alpha$ values) for $\mathrm{Cs}^{+}$against $\mathrm{Na}^{+}$in the presence of $\mathrm{Cl}^{-}$(part A) and $\mathrm{Br}^{-}$(Figures 2D and 2G, in main text). 

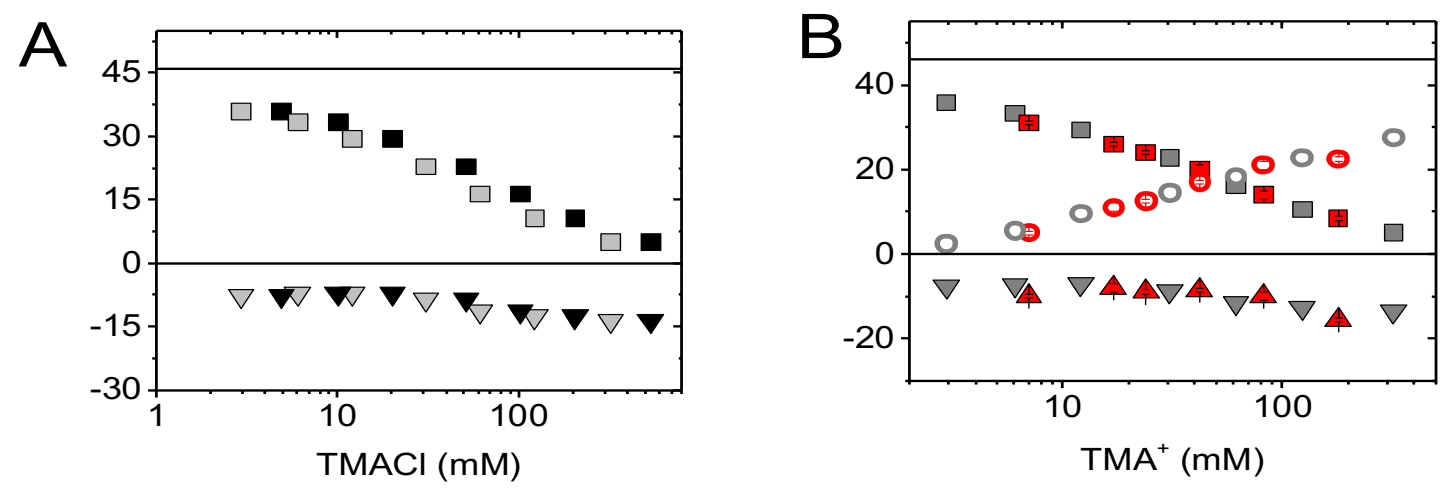

Figure S19. Comparison of prior experimental results (ref 1) of $\mathrm{TMA}^{+}$and $\mathrm{Na}^{+}$competition around 24-bp DNA. A) The number of $\mathrm{Na}^{+}$(squares) in the ion atmosphere from ref 1 as a function of TMACl concentration. TMACl concentration reported in ref 1 (black symbols) and recalculated assuming that the TMACl solution was made from the hydrated instead of anhydrous salt (gray symbols; $\mathrm{M}_{\mathrm{w}}=181.6 \mathrm{~g} / \mathrm{mol}$ vs. $\mathrm{M}_{\mathrm{w}}=109.6 \mathrm{~g} / \mathrm{mol}$, as described in the main text). The number of excluded anions are from ref 1 and are represented by inversed triangles. B) Comparison of prior experimental results of TMACl and $\mathrm{Na}^{+}$ competition, with $\mathrm{TMA}^{+}$concentrations estimated based on the mass of hydrated salt (part (A), gray symbols) with present experimental results for TMABr vs. $\mathrm{NaBr}$ competition (red symbols: $\mathrm{Na}^{+}$, squares; $\mathrm{TMA}^{+}$: open circles); excluded anions: anions from ref 1 are represented by inverted triangles, excluded $\mathrm{Br}^{-}$anions measured herein are represented by red triangles. The black solid lines at $\Gamma=+46$ represents the charge needed to neutralize the total DNA charge of -46 . Error bars for data points measured herein (TMABr and $\mathrm{NaBr}$ ) are standard deviations from three independent measurements. 

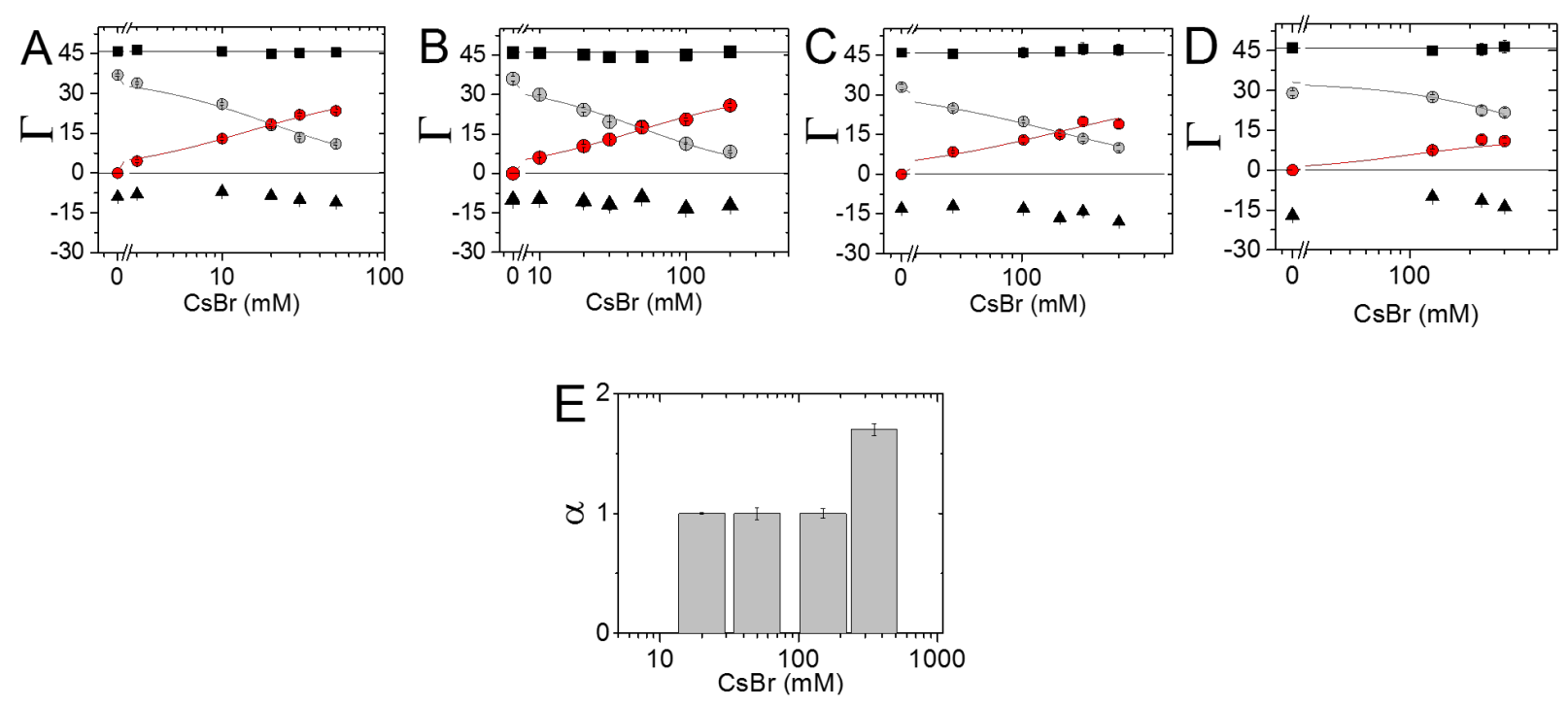

Figure S20. Competitive association of monovalent cations with a 24-bp DNA duplex. The number of ions in the ion atmosphere for $\mathrm{Cs}^{+}$(red circles) versus $\mathrm{Na}^{+}$(gray circles; A) $\left[\mathrm{Na}^{+}\right]=20 \mathrm{mM}, \mathrm{B}$ ) $\left[\mathrm{Na}^{+}\right]=50$ $\left.\mathrm{mM}, \mathrm{C})\left[\mathrm{Na}^{+}\right]=150 \mathrm{mM}, \mathrm{D}\right)\left[\mathrm{Na}^{+}\right]=300 \mathrm{mM}$ ); excluded $\mathrm{Br}^{-}$anion is represented by the black triangles, and the total charge of the ion atmosphere summed from the individual ion measurements is shown as the black squares. The black solid lines at $\Gamma=+46$ represent the charge needed to neutralize the total DNA charge of -46 . Solid lines (red and gray) are fits with the Hill equation and provide an empirical guide. For A), B), C) and D), errors are the standard deviation of all measurements. Data from Tables S4, S11S13. E) Cation competition constants for $\mathrm{Cs}^{+}$against $\mathrm{Na}^{+}$. Each data point in panels $\mathrm{A}$ and $\mathrm{B}$ is the average of three independent measurements. 

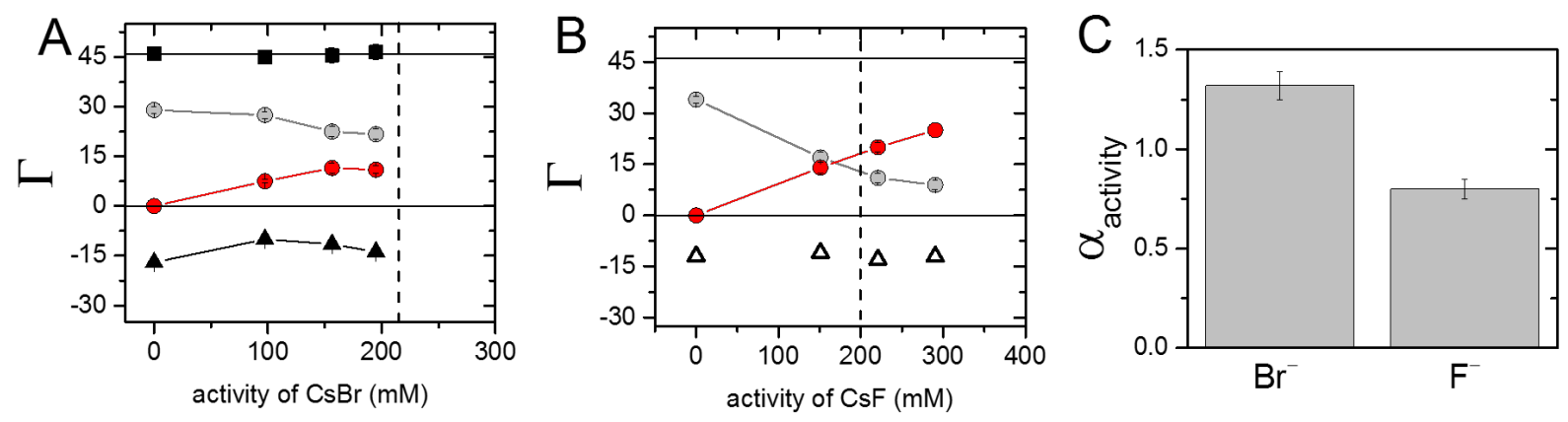

Figure S21. Competitive association of monovalent cations with a 24-bp DNA duplex as a function of the background salt activity. A) Competitive association of $\mathrm{CsBr}$ against $\mathrm{NaBr}$. The number of ions in the ion atmosphere $\mathrm{Cs}^{+}$(red circles) versus $\mathrm{Na}^{+}$(gray circles); excluded $\mathrm{Br}^{-}$anions are represented by the black triangles, and the total charge of the ion atmosphere summed from the individual ion measurements is shown as the black squares. B) Competitive association of $\mathrm{CsF}$ against $\mathrm{NaF}$. The number of ions in the ion atmosphere $\mathrm{Cs}^{+}$(red circles) versus $\mathrm{Na}^{+}$(gray circles); excluded $\mathrm{F}^{-}$anions are represented by the open triangles and were estimated based on eq 1 (in main text). In A) and B), the vertical dashed lines represent the activity of background salt. The black solid lines at $\Gamma=+46$ represent the charge needed to neutralize the total DNA charge of -46 . Each data point is the average of 3-5 independent measurements. Errors are the standard deviation of all measurements. Mean activity of solutions was estimated as described in "Conversion of molar concentration units to molal concentration units and calculation of mean ionic activity of monovalent salts," page S3. C) Cation competition constants for $\mathrm{Cs}^{+}$against $\mathrm{Na}^{+}$as a function of the accompanying anion. The $\alpha_{\text {activiy }}$ value (eq 5), for $\mathrm{Cs}^{+}$versus $\mathrm{Na}^{+}$in the presence of $\mathrm{Br}^{-}$ is higher than 1 , indicating a lower occupancy of $\mathrm{Cs}^{+}$compared to $\mathrm{Na}^{+}$. In contrast, the $\alpha_{\text {activiy }}$ value, for $\mathrm{Cs}^{+}$versus $\mathrm{Na}^{+}$in the presence of $\mathrm{F}^{-}$anion is lower than 1, indicating preferential occupancy of $\mathrm{Cs}^{+}$over $\mathrm{Na}^{+}$. See Tables S13 and S14 in Supplementary Information for raw data. 

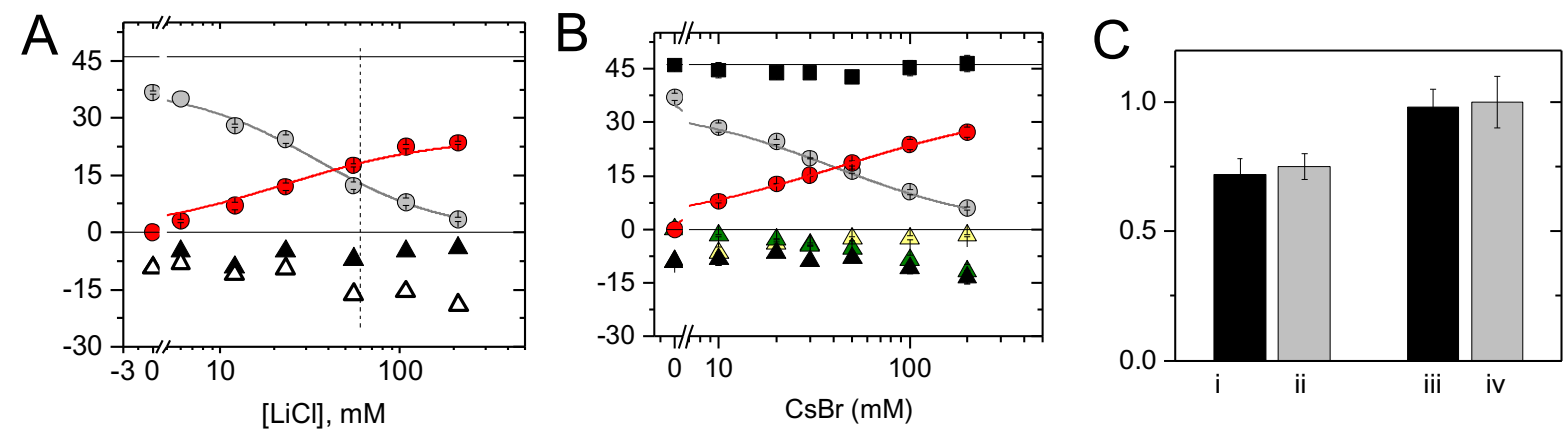

Figure S22. Competitive association of monovalent cations with a 24-bp DNA duplex as a function of anions at the low bulk concentration. A) The number of ions in the ion atmosphere for the competition of $\mathrm{LiCl}$ against $\mathrm{NaO}_{2} \mathrm{As}\left(\mathrm{CH}_{3}\right)_{2}: \mathrm{Li}^{+}$(red circles), $\mathrm{Na}^{+}$(gray circles, $\left[\mathrm{Na}^{+}\right]=50 \mathrm{mM}$ ), excluded $\left(\mathrm{CH}_{3}\right)_{2} \mathrm{AsO}_{2}{ }^{-}$ anion (black triangles), the estimated total number of excluded anions based on eq 1 (in main text) are shown by open black triangles. B) The number of ions in the ion atmosphere for the competition of $\mathrm{CsBr}$ against $\mathrm{NaO}_{2} \mathrm{As}\left(\mathrm{CH}_{3}\right)_{2}: \mathrm{Cs}^{+}$(red circles), $\mathrm{Na}^{+}$(gray circles, $\left[\mathrm{Na}^{+}\right]=40 \mathrm{mM}$ ), excluded $\left(\mathrm{CH}_{3}\right)_{2} \mathrm{AsO}_{2}{ }^{-}$anion (yellow triangles), excluded $\mathrm{Br}^{-}$(green triangles) the estimated total number of excluded anions by summing up the number of $\left(\mathrm{CH}_{3}\right)_{2} \mathrm{AsO}_{2}{ }^{-}$and $\mathrm{Br}^{-}$(black triangles). The total charge of the ion atmosphere calculated from the individual ion measurements is shown as black squares. In A) and B) The black solid lines at $\Gamma=+46$ represent the charge needed to neutralize the total DNA charge of -46 . Solid lines (red and gray) are fits with the Hill equation to provide an empirical guide. Each data point in panels A and B is the average of 3-5 independent measurements. Error bars are standard deviation of all measurement. See Tables S19 and S20 herein for raw data. C) Cation competition constants for $\mathrm{Li}^{+}$and $\mathrm{Cs}^{+}$against $\mathrm{Na}^{+}$ as a function of the anion type: i) $\mathrm{LiBr}$ competing with $\mathrm{NaBr}$, ii) $\mathrm{LiCl}$ competing with $\mathrm{NaO}_{2} \mathrm{As}\left(\mathrm{CH}_{3}\right)_{2}$, iii) CsBr competition with $\mathrm{NaBr}$, iv) $\mathrm{CsBr}$ competing with $\mathrm{NaO}_{2} \mathrm{As}\left(\mathrm{CH}_{3}\right)_{2}$. 

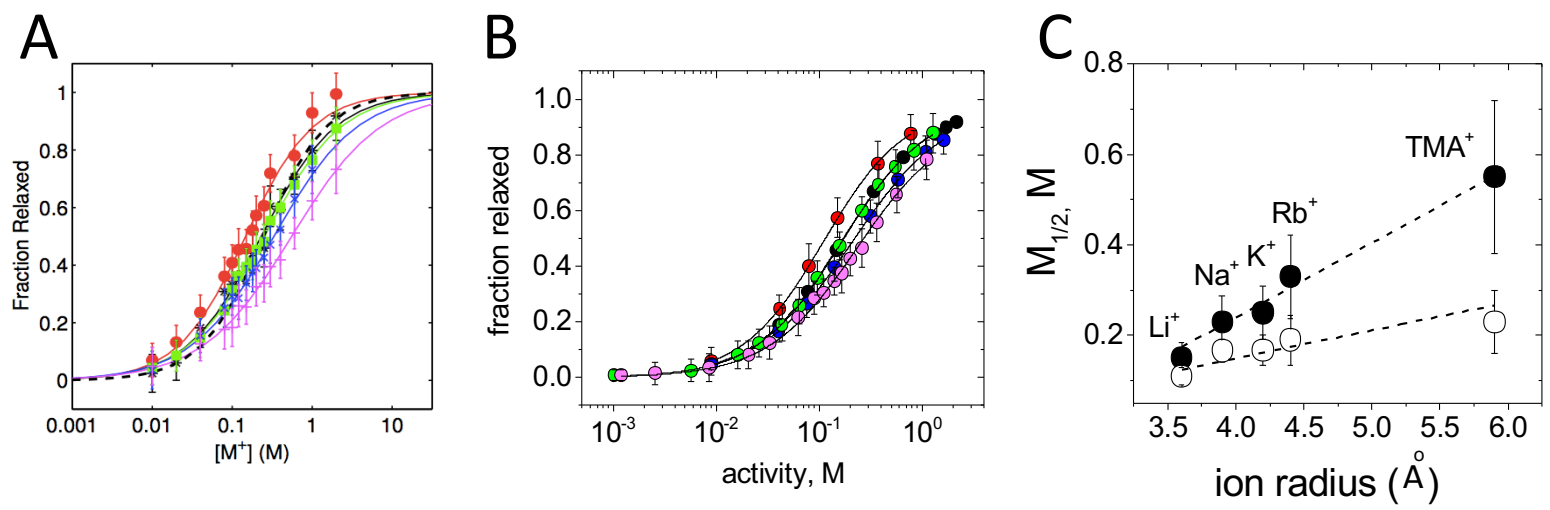

Figure S23. Salt-mediated structural transition of a construct with two DNA helices linked by a PEG tether from ref 3. A) Structural transition of the tethered construct, referred here as a helix-junction-helix $(\mathrm{HJH})$, as a function of monovalent salt concentrations: $\mathrm{LiCl}$ (red), $\mathrm{NaCl}$ (black), $\mathrm{KCl}$ (green), $\mathrm{RbCl}$ (blue), TMACl (magenta). Solid lines are fits with the Hill equation to provide an empirical description of the data. Detailed description of data assessment in ref 3. Reprinted from ref 3. B) Structural transition of the $\mathrm{HJH}$ construct as a function of mean activity of monovalent salts; data from part A, with concentrations converted to activities (see "Conversion of molar concentration units to molal concentration units and calculation of mean ionic activity of monovalent salts", page S3): $\mathrm{LiCl}$ (red), $\mathrm{NaCl}$ (black), $\mathrm{KCl}$ (green), $\mathrm{RbCl}$ (blue), $\mathrm{TMACl}$ (magenta). Solid lines are fits to the Hill equation. C) Comparison of relaxation midpoints obtained from fits with Hill equation from part A (concentration based; closed symbols) and part B (activity based; open symbols). 


\section{Supporting Tables}

Table S1: Experimentally determined preferential interaction coefficients and $\alpha$ value for $\mathbf{L i B r}$ around 24-bp DNA in the presence of $40 \mathrm{mM} \mathrm{NaBr}$.

\begin{tabular}{c|ccc||c||c}
\hline $\mathbf{C}[\mathbf{M}]$ & $\boldsymbol{\Gamma}_{\boldsymbol{L i}}$ & $\boldsymbol{\Gamma}_{\boldsymbol{N} \boldsymbol{a}^{+}}$ & $\boldsymbol{\Gamma}_{\boldsymbol{B} \boldsymbol{r}^{-}}$ & total & $\boldsymbol{\alpha}^{\mathbf{a}}$ \\
\hline 0.000 & 0 & $36.5 \pm 1.0$ & $-10 \pm 1.0$ & $46.5 \pm 1.4$ & \\
0.007 & $6.0 \pm 0.2$ & $30.5 \pm 0.5$ & $-10 \pm 0.5$ & $46.5 \pm 0.7$ & \\
0.017 & $12.0 \pm 0.4$ & $23.5 \pm 0.5$ & $-11 \pm 0.5$ & $46.5 \pm 0.8$ & $0.72 \pm 0.04$ \\
0.051 & $21.0 \pm 0.5$ & $14.5 \pm 0.3$ & $-11 \pm 0.3$ & $46.5 \pm 0.6$ & \\
0.100 & $26.5 \pm 0.5$ & $7.5 \pm 0.3$ & $-12 \pm 1.0$ & $46.0 \pm 1.15$ & \\
0.200 & $30.5 \pm 0.5$ & $4.0 \pm 0.2$ & $-12 \pm 0.5$ & $46.5 \pm 0.7$ & \\
\hline
\end{tabular}

a) $\alpha \overline{\frac{\beta}{[B G]}}$, where $\beta$ is the competition constant of the competing cation (see Experimental Methods and ref 1 ) and [BG] is the concentration of the background cation, $\mathrm{Na}^{+}$.

Table S2: Experimentally determined preferential interaction coefficients and $\alpha$ value for $\mathbf{K B r}$ around 24-bp DNA in the presence of $50 \mathrm{mM} \mathrm{NaBr}$.

\begin{tabular}{c||ccc||c||c}
\hline $\mathbf{C}[\mathbf{M}]$ & $\boldsymbol{\Gamma}_{\boldsymbol{K}^{+}}$ & $\boldsymbol{\Gamma}_{\boldsymbol{N \boldsymbol { a } ^ { + }}}$ & $\boldsymbol{\Gamma}_{\boldsymbol{B \boldsymbol { r } ^ { - }}}$ & total & $\boldsymbol{\alpha}^{\mathbf{a}}$ \\
\hline 0.000 & 0 & $36.0 \pm 0.5$ & $-10.0 \pm 1.0$ & $46.0 \pm 1.1$ & \\
0.011 & $5.0 \pm 0.4$ & $30.0 \pm 0.4$ & $-11.0 \pm 0.7$ & $46.0 \pm 0.9$ & \\
0.021 & $11.0 \pm 0.6$ & $25.0 \pm 0.5$ & $-9.0 \pm 1.5$ & $45.0 \pm 1.2$ & $0.94 \pm 0.07$ \\
0.054 & $18.0 \pm 0.5$ & $19.0 \pm 0.7$ & $-9.0 \pm 0.6$ & $46.0 \pm 1.0$ & \\
0.110 & $25.0 \pm 0.3$ & $12.0 \pm 1.0$ & $-9.0 \pm 0.8$ & $46.0 \pm 1.3$ & \\
0.220 & $29.0 \pm 1.0$ & $5.0 \pm 0.5$ & $-13.0 \pm 1.0$ & $47.0 \pm 1.5$ & \\
\hline
\end{tabular}

a) $\alpha \overline{\frac{\beta}{[B G]}}$, where $\beta$ is the competition constant of the competing cation (see Experimental Methods and ref 1) and [BG] is the concentration of the background cation, $\mathrm{Na}^{+}$.

Table S3: Experimentally determined preferential interaction coefficients and $\alpha$ value for $\mathbf{R b B r}$ around 24-bp DNA in the presence of $50 \mathrm{mM} \mathrm{NaBr}$.

\begin{tabular}{c||ccc||c||c}
\hline $\mathbf{C}[\mathbf{M}]$ & $\boldsymbol{\Gamma}_{\boldsymbol{R} \boldsymbol{b}^{+}}$ & $\boldsymbol{\Gamma}_{\boldsymbol{N \boldsymbol { a } ^ { + }}}$ & $\boldsymbol{\Gamma}_{\boldsymbol{B} \boldsymbol{r}^{-}}$ & total & $\boldsymbol{\alpha}^{\mathbf{a}}$ \\
\hline 0.000 & 0 & $36.0 \pm 0.5$ & $-10.0 \pm 0.5$ & $46.0 \pm 0.5$ & \\
0.009 & $5.0 \pm 0.4$ & $29.2 \pm 0.3$ & $-9.3 \pm 0.7$ & $44.5 \pm 0.8$ & \\
0.019 & $11.0 \pm 0.6$ & $25.4 \pm 0.4$ & $-10.8 \pm 1.1$ & $45.2 \pm 1.4$ & $0.99 \pm 0.05$ \\
0.048 & $18.0 \pm 0.5$ & $16.7 \pm 0.2$ & $-11.7 \pm 0.1$ & $45.4 \pm 0.2$ & \\
0.96 & $25.0 \pm 0.3$ & $11.3 \pm 0.3$ & $-12.2 \pm 0.5$ & $45.3 \pm 0.6$ & \\
0.19 & & $7.5 \pm 1.0$ & $-11.4 \pm 1.0$ & $47.0 \pm 0.7$ & \\
\hline
\end{tabular}

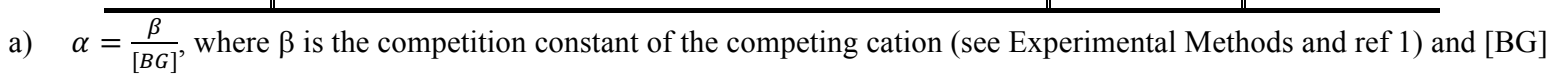
is the concentration of the background cation, $\mathrm{Na}^{+}$. 
Table S4: Experimentally determined preferential interaction coefficients and $\alpha$ value for $\mathbf{C s B r}$ around 24-bp DNA in the presence of $50 \mathrm{mM} \mathrm{NaBr}$.

\begin{tabular}{c||ccc||c||c}
\hline $\mathbf{C}[\mathbf{M}]$ & $\boldsymbol{\Gamma}_{\boldsymbol{C \boldsymbol { s } ^ { + }}}$ & $\boldsymbol{\Gamma}_{\boldsymbol{N a}^{+}}$ & $\boldsymbol{\Gamma}_{\boldsymbol{B \boldsymbol { r } ^ { - }}}$ & total & $\boldsymbol{\alpha}^{\mathbf{a}}$ \\
\hline 0.000 & 0 & $36.0 \pm 0.5$ & $-10.0 \pm 0.5$ & $46.0 \pm 0.5$ & \\
0.01 & $6.0 \pm 0.3$ & $30.0 \pm 0.1$ & $-9.8 \pm 0.2$ & $45.8 \pm 0.2$ & \\
0.02 & $10.5 \pm 0.6$ & $24.2 \pm 0.8$ & $-11.6 \pm 1.0$ & $46.2 \pm 1.4$ & $0.96 \pm 0.03$ \\
0.03 & $13.0 \pm 0.1$ & $19.7 \pm 0.1$ & $-12.7 \pm 1.4$ & $45.0 \pm 1.2$ & \\
0.05 & $17.5 \pm 0.2$ & $17.8 \pm 0.3$ & $-9 \pm 0.5$ & $45.3 \pm 0.6$ & \\
0.10 & $20.5 \pm 0.7$ & $11.3 \pm 0.4$ & $-13.4 \pm 1.0$ & $45.0 \pm 1.3$ & \\
0.2 & $26 \pm 0.75$ & $8.2 \pm 0.4$ & $-12.0 \pm 1.0$ & $46.2 \pm 1.3$ & \\
\hline
\end{tabular}

a) $\alpha \overline{\frac{\beta}{[B G]}}$, where $\beta$ is the competition constant of the competing cation (see Experimental Methods and ref 1) and [BG] is the concentration of the background cation, $\mathrm{Na}^{+}$.

Table S5: Experimentally determined preferential interaction coefficients and $\alpha$ value for $\mathbf{T M A B r}$ around 24-bp DNA in the presence of $50 \mathrm{mM} \mathrm{NaBr}$.

\begin{tabular}{c||ccc||c||c}
\hline $\mathbf{C}[\mathbf{M}]^{\mathbf{a}}$ & $\boldsymbol{\Gamma}_{\boldsymbol{T M A ^ { + }}}{ }^{\mathbf{b}}$ & $\boldsymbol{\Gamma}_{\boldsymbol{N a} \boldsymbol{a}^{+}}$ & $\boldsymbol{\Gamma}_{\boldsymbol{B} \boldsymbol{r}^{-}}$ & total & $\boldsymbol{\alpha}^{\mathbf{d}}$ \\
\hline 0.000 & 0 & $36.5 \pm 0.5$ & $-9.0 \pm 0.5$ & $45.5 \pm 0.5$ & \\
0.007 & $5.0 \pm 0.5$ & $31.0 \pm 0.5$ & $-10.0 \pm 0.5$ & $46^{\mathrm{c}}$ & \\
0.017 & $11.0 \pm 1.0$ & $26.0 \pm 0.5$ & $-8.0 \pm 1.0$ & $46^{\mathrm{c}}$ & $0.95 \pm 0.05$ \\
0.024 & $12.5 \pm 0.5$ & $24.0 \pm 0.5$ & $-9.0 \pm 1.4$ & $46^{\mathrm{c}}$ & \\
0.042 & $17.0 \pm 0.5$ & $20.0 \pm 1.0$ & $-8.5 \pm 0.5$ & $46^{\mathrm{c}}$ & \\
0.082 & $21.0 \pm 1.0$ & $14.0 \pm 1.0$ & $-10.0 \pm 1.0$ & $46^{\mathrm{c}}$ & \\
0.180 & $23.0 \pm 0.5$ & $8.5 \pm 0.5$ & $-14.5 \pm 0.5$ & $46^{\mathrm{c}}$ & \\
\hline
\end{tabular}

a) Determined from Br concentration assayed by ICP MS.

b) Determined from overall charge neutrality (eq 1).

c) Estimated based on the neutrality principle and the charge of nucleic acid (eq 1).

d) $\alpha=\frac{\beta}{[B G]}$, where $\beta$ is the competition constant of the competing cation (see Experimental Methods and ref 1) and [BG] is the concentration of the background cation, $\mathrm{Na}^{+}$. 
Table S6: Experimentally determined preferential interaction coefficients and $\alpha$ value for TBABr around 24-bp DNA in the presence of $40 \mathrm{mM} \mathrm{NaBr}$.

\begin{tabular}{c||ccc||c||c}
\hline $\mathbf{C}[\mathbf{M}]^{\mathbf{a}}$ & $\boldsymbol{\Gamma}_{\boldsymbol{T B A ^ { + }}}{ }^{\mathbf{b}}$ & $\boldsymbol{\Gamma}_{\boldsymbol{N a} \boldsymbol{a}^{+}}$ & $\boldsymbol{\Gamma}_{\boldsymbol{B} \boldsymbol{r}^{-}}$ & total & $\boldsymbol{\alpha}^{\mathbf{d}}$ \\
\hline 0.000 & 0 & $36.7 \pm 1.0$ & $-10.0 \pm 0.5$ & $46.7 \pm 0.5$ & \\
0.005 & $4.0 \pm 1.0$ & $34.0 \pm 0.1$ & $-9.0 \pm 1.0$ & $46^{\mathrm{c}}$ & \\
0.010 & $6.0 \pm 1.0$ & $34.0 \pm 1.5$ & $-6.0 \pm 1.0$ & $46^{\mathrm{c}}$ & $>2.8 \pm 0.25$ \\
0.036 & $11.5 \pm 1.0$ & $25.0 \pm 0.5$ & $-9.0 \pm 1.0$ & $46^{\mathrm{c}}$ & \\
0.070 & $11.0 \pm 0.5$ & $22.0 \pm 0.5$ & $-12.0 \pm 0.5$ & $46^{\mathrm{c}}$ & \\
0.170 & $20.0 \pm 1.0$ & $17.0 \pm 0.5$ & $-18.0 \pm 1.0$ & $46^{\mathrm{c}}$ & \\
\hline
\end{tabular}

a) Determined from Br concentration assayed by ICP MS.

b) Determined from overall charge neutrality (eq 1).

c) Estimated based on the neutrality principle and the charge of nucleic acid (eq 1).

d) $\quad \alpha=\frac{\beta}{[B G]}$, where $\beta$ is the competition constant of the competing cation (see Experimental Methods and ref 1) and [BG] is the concentration of the background cation, $\mathrm{Na}^{+}$.

Table S7: Summary of experimentally determined cation competition constant, $\alpha$ value, against $\mathrm{NaBr}$ around 24-bp DNA (Table S1-S6).

\begin{tabular}{c||c}
\hline salt & $\boldsymbol{\alpha}$ \\
\hline $\mathrm{LiBr}$ & $0.72 \pm 0.04$ \\
$\mathrm{KBr}$ & $0.94 \pm 0.07$ \\
$\mathrm{RbBr}$ & $0.99 \pm 0.05$ \\
$\mathrm{CsBr}$ & $0.96 \pm 0.03$ \\
$\mathrm{TMABr}$ & $0.95 \pm 0.05$ \\
$\mathrm{TBABr}$ & $>2.8 \pm 0.25$ \\
\hline
\end{tabular}

Table S8: Experimentally determined preferential interaction coefficients for $\mathbf{L i B r}$ around 24-bp DNA at difference salt concentrations.

\begin{tabular}{c||cc||c}
\hline $\mathbf{C}[\mathbf{M}]^{\mathbf{a}}$ & $\boldsymbol{\Gamma}_{\boldsymbol{L} \boldsymbol{i}^{+}}$ & $\boldsymbol{\Gamma}_{\boldsymbol{B} \boldsymbol{r}^{-}}$ & total \\
\hline 0.01 & $40.0 \pm 0.5$ & $-6.0 \pm 0.5$ & $46.5 \pm 1.1$ \\
0.020 & $41.0 \pm 0.5$ & $-6.0 \pm 0.5$ & $46.0 \pm 0.7$ \\
0.040 & $39.0 \pm 0.5$ & $-8.0 \pm 1.0$ & $48.0 \pm 1.1$ \\
0.084 & $37.5 \pm 0.5$ & $-10.5 \pm 0.5$ & $45.5 \pm 0.7$ \\
0.175 & $35.5 \pm 1.0$ & $-12.0 \pm 1.0$ & $45.8 \pm 1.4$ \\
0.270 & $34.0 \pm 1.5$ & $-13.0 \pm 1.5$ & $46.0 \pm 2.1$ \\
\hline
\end{tabular}

a) Determined from $\mathrm{Br}$ concentration assayed by ICP MS. 
Table S9: Experimentally determined preferential interaction coefficients and $\alpha$ value for $\mathbf{N a B r}$ around 24-bp DNA in the presence of $16 \mathrm{mM} \mathrm{LiBr}$.

\begin{tabular}{c||ccc||c||c}
\hline $\mathbf{C}[\mathbf{M}]$ & $\boldsymbol{\Gamma}_{\boldsymbol{N \boldsymbol { a } ^ { + }}}$ & $\boldsymbol{\Gamma}_{\boldsymbol{L i}}$ & $\boldsymbol{\Gamma}_{\boldsymbol{B \boldsymbol { r } ^ { - }}}$ & total & $\boldsymbol{\alpha}^{\mathbf{a}}$ \\
\hline 0.000 & 0 & $40 \pm 0.5$ & $-6.5 \pm 0.5$ & $46.5 \pm 0.5$ & \\
0.005 & $7.5 \pm 0.2$ & $29.0 \pm 0.5$ & $-9.5 \pm 0.5$ & $46.0 \pm 1.1$ & \\
0.010 & $11.5 \pm 1.0$ & $25.0 \pm 0.2$ & $-10.2 \pm 0.5$ & $46.7 \pm 1.0$ & \\
0.020 & $17.5 \pm 0.5$ & $17.5 \pm 0.5$ & $-11.0 \pm 0.2$ & $46.0 \pm 0.9$ & $1.25 \pm 0.08$ \\
0.030 & $19.8 \pm 0.3$ & $13.3 \pm 0.1$ & $-12.6 \pm 0.3$ & $45.7 \pm 0.8$ & \\
0.041 & $21.5 \pm 0.5$ & $11.0 \pm 0.5$ & $-12.0 \pm 0.5$ & $44.5 \pm 1.2$ & \\
0.051 & $24.0 \pm 0.5$ & $9.0 \pm 0.2$ & $-12.5 \pm 0.5$ & $45.5 \pm 1.1$ & \\
\hline
\end{tabular}

a) $\alpha \overline{\frac{\beta}{[B G]}}$, where $\beta$ is the competition constant of the competing cation (see Experimental Methods and ref 1) and [BG] is the concentration of the background cation, $\mathrm{Li}^{+}$.

Table S10: Experimentally determined preferential interaction coefficients and $\alpha$ value for $\mathbf{N a B r}$ around 24-bp DNA in the presence of $50 \mathrm{mM} \mathrm{LiBr}$.

\begin{tabular}{c||ccc||c||c}
\hline $\mathbf{C}[\mathbf{M}]$ & $\boldsymbol{\Gamma}_{\boldsymbol{N \boldsymbol { a } ^ { + }}}$ & $\boldsymbol{\Gamma}_{\boldsymbol{L i}^{+}}$ & $\boldsymbol{\Gamma}_{\boldsymbol{B \boldsymbol { r } ^ { - }}}$ & total & $\boldsymbol{\alpha}^{\mathbf{a}}$ \\
\hline 0.000 & 0 & $39 \pm 0.5$ & $-7.5 \pm 0.5$ & $46.5 \pm 0.7$ & \\
0.020 & $10.0 \pm 0.3$ & $30.0 \pm 1.5$ & $-6.0 \pm 0.5$ & $46.0 \pm 1.6$ & \\
0.030 & $12.0 \pm 0.5$ & $23.0 \pm 1.0$ & $-13.0 \pm 1.0$ & $48.0 \pm 1.5$ & $1.20 \pm 0.07$ \\
0.054 & $15.0 \pm 0.5$ & $19.5 \pm 1.0$ & $-11.0 \pm 1.0$ & $45.5 \pm 1.5$ & \\
0.065 & $16.3 \pm 0.3$ & $18.0 \pm 0.5$ & $-11.6 \pm 1.5$ & $45.8 \pm 1.6$ & \\
0.092 & $17.0 \pm 1.0$ & $12.5 \pm 1.0$ & $-16.5 \pm 0.5$ & $46.0 \pm 1.5$ & \\
0.120 & $18.0 \pm 1.0$ & $11.5 \pm 1.0$ & $-18.0 \pm 1.0$ & $47.5 \pm 1.7$ & \\
\hline
\end{tabular}

a) $\alpha \overline{\frac{\beta}{[B G]}}$, where $\beta$ is the competition constant of the competing cation (see Experimental Methods and ref 1) and [BG] is the concentration of the background cation, $\mathrm{Li}^{+}$.

Table S11: Experimentally determined preferential interaction coefficients and $\alpha$ value for $\mathbf{C s B r}$ around 24-bp DNA in the presence of $20 \mathrm{mM} \mathrm{NaBr}$.

\begin{tabular}{c|ccc||c||c}
\hline $\mathbf{C}[\mathbf{M}]$ & $\boldsymbol{\Gamma}_{\boldsymbol{C s ^ { + }}}$ & $\boldsymbol{\Gamma}_{\boldsymbol{N \boldsymbol { a } ^ { + }}}$ & $\boldsymbol{\Gamma}_{\boldsymbol{B r ^ { - }}}$ & total & $\boldsymbol{\alpha}^{\mathbf{a}}$ \\
\hline 0.000 & 0 & $36.5 \pm 0.5$ & $-9.4 \pm 0.5$ & $46.0 \pm 0.5$ & \\
0.003 & $4.5 \pm 0.5$ & $34.0 \pm 0.5$ & $-8.0 \pm 0.5$ & $46.5 \pm 0.7$ & \\
0.010 & $13.0 \pm 0.5$ & $26.0 \pm 0.5$ & $-7.0 \pm 0.5$ & $46.0 \pm 0.7$ & $0.98 \pm 0.07$ \\
0.020 & $18.5 \pm 0.7$ & $18.0 \pm 0.5$ & $-8.5 \pm 1.0$ & $45.0 \pm 1.2$ & \\
0.030 & $22.50 \pm 0.5$ & $13.4 \pm 0.5$ & $-10.0 \pm 1.0$ & $45.4 \pm 1.6$ & \\
0.050 & $23.5 \pm 0.5$ & $11.0 \pm 0.5$ & $-11.0 \pm 1.0$ & $45.5 \pm 1.5$ & \\
\hline
\end{tabular}

a) $\quad \alpha \overline{\frac{\beta}{[B G]}}$, where $\beta$ is the competition constant of the competing cation (see Experimental Methods and ref 1 ) and [BG] is the concentration of the background cation, $\mathrm{Na}^{+}$. 
Table S12: Experimentally determined preferential interaction coefficients and $\alpha$ value for $\mathbf{C s B r}$ around 24-bp DNA in the presence of $150 \mathrm{mM} \mathrm{NaBr}$.

\begin{tabular}{c|ccc||c||c}
\hline $\mathbf{C}[\mathbf{M}]$ & $\boldsymbol{\Gamma}_{\boldsymbol{C \boldsymbol { s } ^ { + }}}$ & $\boldsymbol{\Gamma}_{\boldsymbol{N \boldsymbol { a } ^ { + }}}$ & $\boldsymbol{\Gamma}_{\boldsymbol{B \boldsymbol { r } ^ { - }}}$ & total & $\boldsymbol{\alpha}^{\mathbf{a}}$ \\
\hline 0.000 & 0 & $33.0 \pm 1.0$ & $-13.0 \pm 1.0$ & $46.0 \pm 1.4$ & \\
0.020 & $4.5 \pm 0.5$ & $31.0 \pm 1.0$ & $-10.0 \pm 1.0$ & $46.5 \pm 1.5$ & \\
0.046 & $13.0 \pm 0.5$ & $25.0 \pm 1.0$ & $-12.0 \pm 1.0$ & $45.5 \pm 1.5$ & \\
0.100 & $18.5 \pm 0.7$ & $20.0 \pm 0.5$ & $-13.0 \pm 1.5$ & $46.0 \pm 1.8$ & $0.97 \pm 0.06$ \\
0.150 & $22.50 \pm 0.5$ & $15.0 \pm 0.5$ & $-16.5 \pm 0.5$ & $46.4 \pm 0.8$ & \\
0.200 & $23.5 \pm 0.5$ & $13.5 \pm 1.0$ & $-14.0 \pm 1.5$ & $47.5 \pm 2.3$ & \\
0.300 & $29.0 \pm 0.5$ & $10.0 \pm 1.0$ & $-18.0 \pm 1.2$ & $47.0 \pm 2.1$ & \\
\hline
\end{tabular}

a) $\quad \alpha \overline{\frac{\beta}{[B G]}}$, where $\beta$ is the competition constant of the competing cation (see Experimental Methods and ref 1) and [BG] is the concentration of the background cation, $\mathrm{Na}^{+}$.

Table S13: Experimentally determined preferential interaction coefficients for CsBr around 24-bp DNA in the presence of $300 \mathrm{mM} \mathrm{NaBr}$.

\begin{tabular}{c||c||ccc||c}
\hline $\mathbf{C}[\mathbf{M}]$ & $\mathbf{a}_{ \pm}[\mathbf{M}]^{\mathbf{a}}$ & $\boldsymbol{\Gamma}_{\boldsymbol{C \boldsymbol { s } ^ { + }}}$ & $\boldsymbol{\Gamma}_{\boldsymbol{N \boldsymbol { a } ^ { + }}}$ & $\boldsymbol{\Gamma}_{\boldsymbol{B} \boldsymbol{r}^{-}}$ & total \\
\hline 0.000 & 0 & 0 & $29.0 \pm 1.0$ & $-17.0 \pm 1.0$ & $46.0 \pm 1.4$ \\
0.130 & 0.975 & $7.5 \pm 0.5$ & $27.5 \pm 1.0$ & $-10.0 \pm 0.5$ & $45.0 \pm 1.7$ \\
0.230 & 0.156 & $11.5 \pm 1.5$ & $22.5 \pm 1.5$ & $-11.5 \pm 1.0$ & $45.5 \pm 2.3$ \\
0.300 & 0.195 & $11.0 \pm 1.1$ & $21.7 \pm 1.5$ & $-13.8 \pm 1.1$ & $46.5 \pm 2.3$ \\
\hline
\end{tabular}

a) Estimated as described in "Conversion of molar concentration units to molal concentration units and calculation of mean ionic activity of monovalent salts" (page S3).

Table S14: Experimentally determined preferential interaction coefficients for CsF around 24-bp DNA in the presence of $300 \mathrm{mM} \mathrm{NaF}$.

\begin{tabular}{c||c||ccc||c}
\hline $\mathbf{C}[\mathbf{M}]$ & $\mathbf{a}_{ \pm}[\mathbf{M}]^{\mathbf{a}}$ & $\boldsymbol{\Gamma}_{\boldsymbol{C} \boldsymbol{s}^{+}}$ & $\boldsymbol{\Gamma}_{\boldsymbol{N \boldsymbol { a } ^ { + }}}$ & $\boldsymbol{\Gamma}_{\boldsymbol{F}^{-}}{ }^{\mathbf{b}}$ & total $^{\mathbf{c}}$ \\
\hline 0.000 & 0 & 0 & $34.0 \pm 1.0$ & $-12.0 \pm 1.0$ & 46 \\
0.200 & 0.151 & $14.0 \pm 1.6$ & $21.0 \pm 1.5$ & $-11.0 \pm 1.0$ & 46 \\
0.300 & 0.221 & $22.0 \pm 1.3$ & $11.0 \pm 1.4$ & $-13.0 \pm 1.0$ & 46 \\
0.400 & 0.290 & $25.0 \pm 2.0$ & $8.9 \pm 1.3$ & $-12.0 \pm 1.5$ & 46 \\
\hline
\end{tabular}

a) Estimated as described in "Conversion of molar concentration units to molal concentration units and calculation of mean ionic activity of monovalent salts" (page S3).

b) Determined based on the overall charge neutrality (eq 1).

c) Estimated based on the neutrality principle and the charge of nucleic acid (eq 1). 
Table S15: Experimentally determined preferential interaction coefficients and $\alpha$ value for $\mathbf{L i B r}$ around 24-bp DNA in the presence of $5 \mathrm{mM} \mathrm{MgBr}_{2}$.

\begin{tabular}{c||ccc||c||c}
\hline $\mathbf{C}[\mathbf{M}]$ & $\boldsymbol{\Gamma}_{\boldsymbol{N \boldsymbol { a } ^ { + }}}$ & $\boldsymbol{\Gamma}_{\boldsymbol{M \boldsymbol { g } ^ { 2 + }}}$ & $\boldsymbol{\Gamma}_{\boldsymbol{B \boldsymbol { r } ^ { - }}}$ & total & $\boldsymbol{\alpha}^{\mathrm{a}}$ \\
\hline 0.000 & 0 & $21.0 \pm 0.3$ & $-4.5 \pm 1.0$ & $46.5 \pm 0.6$ & \\
0.014 & $6.7 \pm 0.5$ & $19.0 \pm 0.5$ & $-2.5 \pm 0.5$ & $47.2 \pm 0.8$ & \\
0.025 & $9.0 \pm 0.3$ & $17.0 \pm 0.5$ & $-5.0 \pm 0.4$ & $48.0 \pm 0.7$ & \\
0.035 & $14.0 \pm 0.5$ & $14.5 \pm 0.5$ & $-3.0 \pm 1.0$ & $46.0 \pm 1.2$ & $7.0 \pm 0.6$ \\
0.06 & $17.5 \pm 0.5$ & $12.5 \pm 0.5$ & $-3.0 \pm 0.5$ & $45.5 \pm 0.8$ & \\
0.110 & $20.0 \pm 1.0$ & $7.5 \pm 0.5$ & $-11.0 \pm 0.5$ & $46.0 \pm 1.2$ & \\
0.220 & $24.5 \pm 0.5$ & $4.5 \pm 0.5$ & $-12.0 \pm 0.5$ & $45.5 \pm 0.9$ & \\
\hline
\end{tabular}

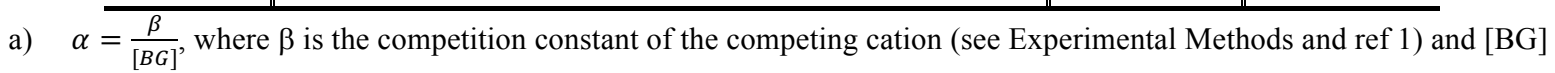
is the concentration of the background cation, $\mathrm{Mg}^{2+}$.

Table S16: Experimentally determined preferential interaction coefficients and $\alpha$ value for $\mathbf{N a B r}$ around 24-bp DNA in the presence of $6 \mathrm{mM} \mathrm{MgBr}_{2}$.

\begin{tabular}{c||ccc||c||c}
\hline $\mathbf{C}[\mathbf{M}]$ & $\boldsymbol{\Gamma}_{\boldsymbol{N a} \boldsymbol{a}^{+}}$ & $\boldsymbol{\Gamma}_{\boldsymbol{M} \boldsymbol{g}^{2+}}$ & $\boldsymbol{\Gamma}_{\boldsymbol{B} \boldsymbol{r}^{-}}$ & total & $\boldsymbol{\alpha}^{\mathrm{a}}$ \\
\hline 0.000 & 0 & $21.5 \pm 053$ & $-3.0 \pm 1.0$ & $46.0 \pm 1.1$ & \\
0.012 & $3.0 \pm 0.2$ & $19.8 \pm 0.2$ & $-3.2 \pm 1.5$ & $45.8 \pm 1.5$ & \\
0.018 & $4.5 \pm 0.5$ & $18.4 \pm 0.5$ & $-5.8 \pm 1.5$ & $47.1 \pm 1.8$ & \\
0.028 & $6.2 \pm 0.1$ & $17.0 \pm 0.1$ & $-4.1 \pm 1.5$ & $44.6 \pm 1.5$ & $8.6 \pm 0.5$ \\
0.048 & $11.6 \pm 0.2$ & $14.0 \pm 0.1$ & $-7.0 \pm 0.5$ & $46.6 \pm 0.5$ & \\
0.100 & $18.0 \pm 0.2$ & $8.6 \pm 0.2$ & $-11.4 \pm 0.5$ & $46.6 \pm 0.6$ & \\
0.200 & $21.0 \pm 1.0$ & $5.8 \pm 0.1$ & $-12.7 \pm 1.5$ & $45.3 \pm 1.8$ & \\
\hline
\end{tabular}

a) $\quad \alpha \overline{\frac{\beta}{[B G]}}$, where $\beta$ is the competition constant of the competing cation (see Experimental Methods and ref 1 ) and [BG] is the concentration of the background cation, $\mathrm{Mg}^{2+}$.

Table S17: Experimentally determined preferential interaction coefficients and $\alpha$ value for $\mathbf{C s B r}$ around 24-bp DNA in the presence of $6 \mathrm{mM} \mathrm{MgBr}_{2}$.

\begin{tabular}{c|ccc||c||c}
\hline $\mathbf{C}[\mathbf{M}]$ & $\boldsymbol{\Gamma}_{\boldsymbol{N \boldsymbol { a } ^ { + }}}$ & $\boldsymbol{\Gamma}_{\boldsymbol{M} \boldsymbol{g}^{2+}}$ & $\boldsymbol{\Gamma}_{\boldsymbol{B \boldsymbol { r } ^ { - }}}$ & total & $\boldsymbol{\alpha}^{\mathrm{a}}$ \\
\hline 0.000 & 0 & $21.5 \pm 0.5$ & $-3.0 \pm 1.0$ & $46.0 \pm 1.1$ & \\
0.004 & $1.6 \pm 0.1$ & $21.0 \pm 0.5$ & $-2.0 \pm 0.5$ & $45.5 \pm 0.7$ & \\
0.014 & $5.1 \pm 0.2$ & $19.5 \pm 0.2$ & $-2.7 \pm 0.1$ & $46.8 \pm 0.2$ & \\
0.020 & $6.1 \pm 0.1$ & $18.4 \pm 0.3$ & $-4.0 \pm 0.3$ & $46.9 \pm 0.5$ & $8.7 \pm 0.8$ \\
0.030 & $9.1 \pm 0.3$ & $16.6 \pm 0.3$ & $-3.1 \pm 0.5$ & $45.4 \pm 0.5$ & \\
0.050 & $13.8 \pm 1.0$ & $13.5 \pm 0.3$ & $-5.5 \pm 1.5$ & $46.3 \pm 2.0$ & \\
0.100 & $18.0 \pm 0.1$ & $9.1 \pm 0.4$ & $-11.4 \pm 0.5$ & $46.9 \pm 1.1$ & \\
0.200 & $20.6 \pm 0.5$ & $6.5 \pm 0.3$ & $-12.7 \pm 1.5$ & $46.3 \pm 0.8$ & \\
\hline
\end{tabular}

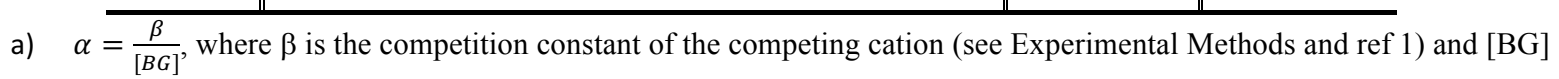
is the concentration of the background cation, $\mathrm{Mg}^{2+}$. 
Table S18: Experimentally determined preferential interaction coefficients and $\alpha$ value for $\mathbf{C s B r}$ around 24-bp RNA in the presence of $50 \mathrm{mM} \mathrm{NaBr}$.

\begin{tabular}{c||ccc||c||c}
\hline $\mathbf{C}[\mathbf{M}]$ & $\boldsymbol{\Gamma}_{\boldsymbol{C s ^ { + }}}$ & $\boldsymbol{\Gamma}_{\boldsymbol{N a ^ { + }}}$ & $\boldsymbol{\Gamma}_{\boldsymbol{B \boldsymbol { r } ^ { - }}}$ & total & $\boldsymbol{\alpha}^{\mathbf{a}}$ \\
\hline 0.012 & $6.0 \pm 0.5$ & $29.0 \pm 0.5$ & $-10.0 \pm 1.0$ & $45.0 \pm 1.2$ & \\
0.025 & $15.5 \pm 0.5$ & $24.0 \pm 1.5$ & $-8.0 \pm 1.5$ & $47.5 \pm 2.0$ & \\
0.061 & $20.0 \pm 1.0$ & $16.5 \pm 1.0$ & $-9.0 \pm 1.0$ & $45.5 \pm 1.7$ & $0.99 \pm 0.08$ \\
0.087 & $24.0 \pm 1.0$ & $14.0 \pm 0.5$ & $-10.0 \pm 0.5$ & $48.0 \pm 1.2$ & \\
0.250 & $30.0 \pm 1.0$ & $5.0 \pm 0.5$ & $-11.1 \pm 1.0$ & $46.0 \pm 1.5$ & \\
\hline
\end{tabular}

a) $\quad \alpha \overline{\frac{\beta}{[B G]}}$, where $\beta$ is the competition constant of the competing cation (see Experimental Methods and ref 1) and [BG] is the concentration of the background cation, $\mathrm{Na}^{+}$.

Table S19: Experimentally determined preferential interaction coefficients and $\alpha$ value for $\mathbf{L i C l}$ around 24-bp DNA in the presence of $60 \mathrm{mM} \mathrm{NaO}_{2} \mathrm{As}\left(\mathrm{CH}_{3}\right)_{2}$.

\begin{tabular}{c||cccc||c||c}
\hline $\mathbf{C}[\mathbf{M}]$ & $\boldsymbol{\Gamma}_{\boldsymbol{L i}}{ }^{+}$ & $\boldsymbol{\Gamma}_{\boldsymbol{N a}}$ & $\boldsymbol{\Gamma}_{\left[\boldsymbol{A s}\left(\boldsymbol{C H}_{2}\right)_{2} \boldsymbol{O}_{2}\right]^{-}}$ & $\boldsymbol{\Gamma}_{\boldsymbol{C l}}{ }^{\mathbf{a}}$ & total & $\boldsymbol{\alpha}^{\mathbf{c}}$ \\
\hline 0.00 & 0.0 & $36.7 \pm 0.5$ & $-9.0 \pm 1.0$ & 0 & $45.7 \pm 1.2$ & \\
0.006 & $3.0 \pm 0.5$ & $35.0 \pm 0.2$ & $-5.0 \pm 3.0$ & -3 & $46^{\mathrm{b}}$ & \\
0.012 & $7.0 \pm 1.0$ & $28.0 \pm 0.5$ & $-9.0 \pm 1.0$ & -2 & $46^{\mathrm{b}}$ & $0.75 \pm 0.05$ \\
0.023 & $12.0 \pm 1.0$ & $24.5 \pm 0.5$ & $-5.0 \pm 0.5$ & -4.5 & $46^{\mathrm{b}}$ & \\
0.055 & $17.6 \pm 0.5$ & $12.2 \pm 1.0$ & $-7.0 \pm 1.2$ & -9.2 & $46^{\mathrm{b}}$ & \\
0.108 & $22.2 \pm 0.5$ & $8.0 \pm 1.0$ & $-5.0 \pm 1.0$ & -10.8 & $46^{\mathrm{b}}$ & \\
0.211 & $23.2 \pm 0.5$ & $3.5 \pm 0.5$ & $-4.2 \pm 1.0$ & -15.1 & $46^{\mathrm{b}}$ & \\
\hline
\end{tabular}

a) Determined from overall charge neutrality (eq 1).

b) Estimated based on the neutrality principle and the charge of nucleic acid (eq 1).

c) $\quad \alpha=\frac{\beta}{[B G]}$, where $\beta$ is the competition constant of the competing cation (see Experimental Methods and ref 1) and [BG] is the concentration of the background cation, $\mathrm{Na}^{+}$.

Table S20. Experimentally determined preferential interaction coefficients and $\alpha$ value for $\mathbf{C s B r}$ around 24-bp DNA in the presence of $40 \mathrm{mM} \mathrm{NaO}_{2} \mathrm{As}\left(\mathrm{CH}_{3}\right)_{2}$.

\begin{tabular}{c||cccc||c||c}
$\mathbf{C}[\mathbf{M}]$ & $\boldsymbol{\Gamma}_{\boldsymbol{C \boldsymbol { s } ^ { + }}}$ & $\boldsymbol{\Gamma}_{\boldsymbol{N a} \boldsymbol{a}^{+}}$ & $\boldsymbol{\Gamma}_{\left[\boldsymbol{A s}\left(\boldsymbol{C H}_{\mathbf{2}}\right)_{2} \boldsymbol{O}_{2}\right]^{-}}$ & $\boldsymbol{\Gamma}_{\boldsymbol{B} \boldsymbol{r}^{-}}{ }^{\mathbf{a}}$ & total & $\boldsymbol{\alpha}^{\mathbf{a}}$ \\
\hline 0.00 & 0.0 & $37.0 \pm 1.0$ & $-9.0 \pm 1.0$ & 0 & $46.0 \pm 1.2$ & \\
0.010 & $7.8 \pm 0.4$ & $28.5 \pm 1.3$ & $-6.5 \pm 1.7$ & $-1.7 \pm 0.3$ & $44.5 \pm 2.2$ & \\
0.030 & $12.7 \pm 0.1$ & $24.5 \pm 0.7$ & $-3.9 \pm 0.3$ & $-2.7 \pm 0.1$ & $44.0 \pm 1.8$ & $1.0 \pm 0.1$ \\
0.030 & $15.1 \pm 0.1$ & $19.9 \pm 0.3$ & $-5.3 \pm 0.5$ & $-4.5 \pm 0.2$ & $45.0 \pm 0.5$ & \\
0.050 & $18.5 \pm 0.7$ & $16.2 \pm 0.6$ & $-4.65 \pm 0.5$ & $-6.3 \pm 1.1$ & $45.6 \pm 1.5$ & \\
0.100 & $23.7 \pm 1.5$ & $10.5 \pm 0.7$ & $-2.4 \pm 0.6$ & $-8.5 \pm 1.3$ & $45.1 \pm 2.2$ & \\
0.200 & $27.2 \pm 1.5$ & $5.9 \pm 0.45$ & $-1.8 \pm 0.3$ & $-11.6 \pm 1.7$ & $46.5 \pm 2.3$ &
\end{tabular}

a) $\quad \alpha=\frac{\beta}{[B G]}$, where $\beta$ is the competition constant of the competing cation (see Experimental Methods and ref 1) and [BG] is the concentration of the background cation, $\mathrm{Na}^{+}$. 
Table S21. Kinetic and thermodynamic parameter for P4-P6 RNA folding in the presence of $\mathrm{NaCl}$.

\begin{tabular}{c||c|c|c|c|c|}
\hline \hline $\begin{array}{c}\mathrm{C} \\
(\mathrm{M})\end{array}$ & $\begin{array}{c}\mathrm{k}_{\text {fold }}^{\mathrm{a}} \\
{\left[\mathrm{s}^{-1}\right]}\end{array}$ & $\begin{array}{c}\mathrm{k}_{\text {unfold }}^{\mathrm{a}} \\
{\left[\mathrm{s}^{-1}\right]}\end{array}$ & $\mathrm{K}_{\mathrm{eq}}^{\mathrm{b}}$ & $\mathrm{K}_{\text {eq,bulk }} \mathrm{c}^{\mathrm{c}}$ & $\mathrm{N}^{\mathrm{d}}$ \\
\hline 1.4 & $1.1 \pm 0.10$ & $18.2 \pm 2.50$ & $0.06 \pm 0.02$ & 0.2 & 68 \\
\hline 1.9 & $1.9 \pm 0.20$ & $13.6 \pm 1.20$ & $0.14 \pm 0.03$ & 0.3 & 86 \\
\hline 2.4 & $4.0 \pm 0.30$ & $9.9 \pm 0.30$ & $0.42 \pm 0.02$ & 0.6 & 93 \\
\hline 2.9 & $5.8 \pm 0.40$ & $6.8 \pm 0.40$ & $0.84 \pm 0.01$ & 1.0 & 106 \\
\hline
\end{tabular}

a) Mean and error are bootstrap-estimated $95 \%$ confidence intervals of the mean.

b) $K_{\text {eq }}=\frac{k_{\text {fold }}}{k_{\text {unfold }}}$.

c) $K_{\text {eq, bulk }}$ determined through a fit of the total FRET distribution of a population of molecules to two-Gaussians (Figure S2) and taken as the ration of the total time spent in the high FRET sate relative to the low FRET state.

d) $\mathrm{N}=$ number of molecules.

Table S22. Kinetic and thermodynamic parameter for P4-P6 RNA folding in the presence of $\mathrm{KCl}$.

\begin{tabular}{c||c|c|c|c|c|}
\hline \hline $\begin{array}{c}\mathrm{C} \\
\mathrm{M})\end{array}$ & $\begin{array}{c}\mathrm{k}_{\text {fold }^{\mathrm{a}}} \\
{\left[\mathrm{s}^{-1}\right]}\end{array}$ & $\begin{array}{c}\mathrm{k}_{\text {unfold }}{ }^{\mathrm{a}} \\
{\left[\mathrm{s}^{-1}\right]}\end{array}$ & $\mathrm{K}_{\mathrm{eq}}^{\mathrm{b}}$ & $\mathrm{K}_{\text {eq,bulk }}{ }^{\mathrm{c}}$ & $\mathrm{N}^{\mathrm{d}}$ \\
\hline 1.6 & $1.0 \pm 0.06$ & $14.0 \pm 1.00$ & $0.07 \pm 0.02$ & 0.2 & 115 \\
\hline 2.0 & $1.9 \pm 0.15$ & $12.0 \pm 0.60$ & $0.16 \pm 0.03$ & 0.3 & 118 \\
\hline 2.5 & $2.8 \pm 0.20$ & $7.4 \pm 0.40$ & $0.38 \pm 0.02$ & 0.4 & 105 \\
\hline 2.8 & $3.8 \pm 0.20$ & $7.1 \pm 0.25$ & $0.53 \pm 0.01$ & 0.6 & 142 \\
\hline
\end{tabular}

a) Mean and error are bootstrap-estimated $95 \%$ confidence intervals of the mean.

b) $K_{\text {eq }}=\frac{k_{\text {fold }}}{k_{\text {unfold }}}$.

c) $K_{\text {eq, bulk }}$ determined through a fit of the total FRET distribution of a population of molecules to two-Gaussians (Figure S2) and taken as the ration of the total time spent in the high FRET sate relative to the low FRET state.

d) $\mathrm{N}=$ number of molecules.

Table S23. Kinetic and thermodynamic parameter for P4-P6 RNA folding in the presence of RbCl.

\begin{tabular}{c||c|c|c|c|c|}
\hline \hline $\begin{array}{c}\mathrm{C} \\
(\mathrm{M})\end{array}$ & $\begin{array}{c}\mathrm{k}_{\text {fold }}^{\mathrm{a}} \\
{\left[\mathrm{s}^{-1}\right]}\end{array}$ & $\begin{array}{c}\mathrm{k}_{\text {unfold }}^{\mathrm{a}} \\
{\left[\mathrm{s}^{-1}\right]}\end{array}$ & $\mathrm{K}_{\mathrm{eq}}^{\mathrm{b}}$ & $\mathrm{K}_{\text {eq,bulk }}{ }^{\mathrm{c}}$ & $\mathrm{N}^{\mathrm{d}}$ \\
\hline 2.0 & $0.85 \pm 0.10$ & $20.5 \pm 1.2$ & $0.04 \pm 0.02$ & 0.1 & 59 \\
\hline 2.4 & $1.43 \pm 0.10$ & $14.7 \pm 0.7$ & $0.09 \pm 0.03$ & 0.1 & 84 \\
\hline \hline 2.8 & $2.2 \pm 0.40$ & $13.0 \pm 0.5$ & $0.17 \pm 0.02$ & 0.1 & 59 \\
\hline
\end{tabular}

a) Mean and error are bootstrap-estimated $95 \%$ confidence intervals of the mean.

b) $K_{\text {eq }}=\frac{k_{\text {fold }}}{k_{\text {unfold }}}$.

c) $K_{\text {eq, bulk }}$ determined through a fit of the total FRET distribution of a population of molecules to two-Gaussians (Figure $\mathrm{S} 2$ ) and taken as the ration of the total time spent in the high FRET sate relative to the low FRET state.

d) $\mathrm{N}=$ number of molecules. 
Table S24. Kinetic and thermodynamic parameter for P4-P6 RNA folding in the presence of RbF.

\begin{tabular}{c||c|c|c|c|c|}
\hline \hline $\begin{array}{c}\mathrm{C} \\
(\mathrm{M})\end{array}$ & $\begin{array}{c}\mathrm{k}_{\text {fold }^{\mathrm{a}}} \\
{\left[\mathrm{s}^{-1}\right]}\end{array}$ & $\begin{array}{c}\mathrm{k}_{\text {unfold }}{ }^{\mathrm{a}} \\
{\left[\mathrm{s}^{-1}\right]}\end{array}$ & $\mathrm{K}_{\text {eq }}{ }^{\mathrm{b}}$ & $\mathrm{K}_{\text {eq,bulk }}{ }^{\mathrm{c}}$ & $\mathrm{N}^{\mathrm{d}}$ \\
\hline 1.8 & $2.2 \pm 0.14$ & $21.5 \pm 1.0$ & $0.10 \pm 0.02$ & 0.2 & 85 \\
\hline 2.3 & $4.0 \pm 0.30$ & $15.0 \pm 0.8$ & $0.26 \pm 0.03$ & 0.3 & 112 \\
\hline 2.5 & $5.6 \pm 0.30$ & $11.7 \pm 0.6$ & $0.48 \pm 0.02$ & 0.6 & 91 \\
\hline
\end{tabular}

a) Mean and error are bootstrap-estimated $95 \%$ confidence intervals of the mean.

b) $K_{\text {eq }}=\frac{k_{\text {fold }}}{k_{\text {unfold }}}$.

c) $K_{\text {eq, bulk }}$ determined through a fit of the total FRET distribution of a population of molecules to two-Gaussians (Figure S2) and taken as the ration of the total time spent in the high FRET sate relative to the low FRET state.

d) $\mathrm{N}=$ number of molecules.

Table S25. Mean activity coefficients $\left(\gamma_{ \pm}\right)$of $\mathrm{NaX}, \mathrm{KCl}, \mathrm{RbX}$ and $\mathrm{CsX}$ salts at $2.0 \mathrm{M}$ and $25^{\circ} \mathrm{C}$

\begin{tabular}{l||c|}
\hline \hline & $\gamma_{ \pm}{ }^{\text {a }}$ \\
\hline $\mathrm{NaCl}$ & 0.668 \\
\hline $\mathrm{NaBr}$ & 0.731 \\
\hline $\mathrm{KCl}$ & 0.573 \\
\hline $\mathrm{RbF}$ & 0.724 \\
\hline $\mathrm{RbCl}$ & 0.546 \\
\hline $\mathrm{CsF}$ & 0.803 \\
\hline $\mathrm{CsBr}$ & 0.486 \\
\hline
\end{tabular}

a) Data from ref 5 .

\section{References:}

(1) Bai, Y.; Greenfeld, M.; Travers, K. J.; Chu, V. B.; Lipfert, J.; Doniach, S.; Herschlag, D. J. Am. Chem. Soc. 2007, 129, 14981.

(2) Gebala, M.; Giambasu, G. M.; Lipfert, J.; Bisaria, N.; Bonilla, S.; Li, G.; York, D. M.; Herschlag, D. J. Am. Chem. Soc. 2015, 137, 14705

(3) Bai, Y.; Chu, V. B.; Lipfert, J.; Pande, V. S.; Herschlag, D.; Doniach, S. J. Am. Chem. Soc. 2008, 130, 12334.

(4) Robinson, R. A.; Stokes, R. H. Electrolyte Solutions; 2nd rev. ed.; Dover Publications: Mineola, NY, 2002.

(5) Hamer, W. J.; Wu, Y. J. Phys. Chem. Ref. Data 1972, 1, 52.

(6) Greenfeld, M.; Pavlichin, D. S.; Mabuchi, H.; Herschlag, D. Plos One 2012, 7, e30024.

(7) Giambasu, G. M.; Gebala, M. K.; Panteva, M. T.; Luchko, T.; Case, D. A.; York, D. M. Nucleic Acids Res. 2015, 43, 8405. 